\title{
Janossy densities for chiral random matrix ensembles and their applications to two-color QCD
}

\author{
Hiroyuki Fuji, $^{a, b}$ Issaku Kanamori $^{c, d}$ and Shinsuke M. Nishigaki ${ }^{e}$ \\ ${ }^{a}$ Faculty of Education, Kagawa University, \\ 1-1 Saiwai-cho, Takamatsu 760-8521, Japan \\ ${ }^{b}$ Centre for Quantum Geometry of Moduli Spaces, Aarhus University, \\ Ny Munkegade 118, DK-8000 Aarhus C, Denmark \\ ${ }^{c}$ Department of Physical Science, Hiroshima University, \\ Higashi-hiroshima 739-8526, Japan \\ ${ }^{d}$ RIKEN Center for Computational Science, \\ Kobe 650-0047, Japan \\ ${ }^{e}$ Department of Physics and Materials Science, \\ Shimane University, Matsue 690-8504 Japan \\ E-mail: fuji@ed.kagawa-u.ac.jp, kanamori-i@riken.jp, \\ mochizuki@riko.shimane-u.ac.jp
}

AbStract: We compute individual distributions of low-lying eigenvalues of massive chiral random matrix ensembles by the Nyström-type quadrature method for evaluating the Fredholm determinant and Pfaffian that represent the analytic continuation of the Janossy densities (conditional gap probabilities). A compact formula for individual eigenvalue distributions suited for precise numerical evaluation by the Nyström-type method is obtained in an explicit form, and the $k^{\text {th }}$ smallest eigenvalue distributions are numerically evaluated for chiral unitary and symplectic ensembles in the microscopic limit. As an application of our result, the low-lying Dirac spectra of the $\mathrm{SU}(2)$ lattice gauge theory with $N_{F}=8$ staggered flavors are fitted to the numerical prediction from the chiral symplectic ensemble, leading to a precise determination of the chiral condensate of a two-color QCD-like system in the future.

KeYwords: Matrix Models, Lattice QCD, Stochastic Processes

ARXIV EPRINT: 1903.07176 


\section{Contents}

1 Introduction $\quad 2$

2 Fredholm determinants and Pfaffians for chiral Gaussian random matrix ensembles

2.1 Gaussian chiral random matrix ensembles and the microscopic limit 4

2.2 Individual eigenvalue distributions 6

2.3 Fredholm determinant for chiral Gaussian unitary ensemble 8

2.4 Fredholm Pfaffian for chiral Gaussian symplectic ensemble 11

3 Numerical evaluation of the Janossy density via the Nyström-type discretization

3.1 Chiral GUE with doubly degenerated masses $N_{F}=2 \alpha$

3.2 Chiral GSE with quadruply degenerated masses $N_{F}=4 \alpha \quad 13$

$\begin{array}{lll}3.3 & \text { Chiral GSE with doubly degenerated masses } N_{F}=2 \alpha & 15\end{array}$

4 Application: chiral condensate from lattice data 18

5 Conclusions and discussions $\quad 22$

A Quaternionic kernels for the chiral Gaussian symplectic ensemble $\quad 24$

A.1 Spectral kernel for quadruply degenerated masses 24

$\begin{array}{lll}\text { A.2 Spectral kernel for doubly degenerated masses } & 25\end{array}$

B Confluent limits of the correlation function $\quad \mathbf{2 6}$

B.1 Chiral Gaussian unitary ensemble 26

B.2 Chiral Gaussian symplectic ensemble 28

B.2.1 Quadruply degenerated kernels in the confluent limit 29

C Janossy density $\quad 30$

C.1 Janossy density for the determinantal random point process 30

C.2 Massive chiral Gaussian ensemble with $N_{F}=\beta n$ fermions and the Janossy density 32

D Probability distribution of the $k^{\text {th }}$ smallest eigenvalue 33

E Gauss-Legendre quadrature rule $\quad 35$

$\begin{array}{ll}\text { F Details of the lattice result } & 37\end{array}$

$\begin{array}{ll}\text { G Estimation of the correlation matrix } & 37\end{array}$

H Hybrid Monte Carlo (HMC) for RMT 38

I Data of $k^{\text {th }}$ smallest eigenvalue distributions for chiral GSE with $N_{F}=8 \quad 39$ 


\section{Introduction}

Random matrix theory (RMT) has served as fundamental tool for analysing quantum spectra of classically chaotic systems. Universality of the level statistics of invariant RMTs provides a basis upon which the system-specific information, due e.g. to the presence of short periodic orbits or to the weak localization effect, may be encoded [1]. In the application of RMT to QCD or gauge theories in general, the focus is on the distributions of several smallest eigenvalues of chiral RM ensembles, as they describe the spectral statistics of gauge-covariant Dirac operators in the broken phase of chiral symmetry. (Examples of such applications are found in [2-6].) This relation is particularly useful with lattice simulations. If a gauge theory is in the chirally broken phase and not in the conformal window, its low-energy excitations are unambiguously described by the chiral Lagrangian on one of the Riemannian symmetric spaces (Nambu-Goldstone manifolds) $\mathcal{M}$. In that case, (i) the low-lying Dirac eigenvalues $0 \leq \lambda_{1} \leq \lambda_{2} \leq \cdots$ measured on lattices of different volumes $V$ will, after prescribed unfolding $x_{k}=\Sigma V \lambda_{k}$ and scaling of quark masses $\mu_{f}=\Sigma V m_{f}$, with a constant $\Sigma$ independent of the volumes, obey a single statistical distribution $p_{k}\left(x ;\left\{\mu_{f}\right\}\right)=\left\langle\delta\left(x-x_{k}\right)\right\rangle$, and (ii) this distribution will be identical to the one from the RMT that is equivalent to the zero-momentum part of the chiral Lagrangian on $\mathcal{M}$ [7]. If the theory is in the symmetric phase of the chiral symmetry, no such scaling with the volume, which collapses the distributions of $\lambda_{k}$ 's from different volumes onto a single function, would appear. Previously this criterion was applied to QCD around the critical temperature, and the inconsistency with RMT (including non-scaling of unfolded Dirac eigenvalues with volumes) was considered as a sign of chiral symmetry restoration [8]. In addition, if the theory is conformal, no scale should appear so that the chiral condensate $\Sigma$ should disappear in the chiral limit and description with RMT is not applicable.

In the proposal of the walking technicolor model [9], the choice of the gauge group of techni-gluons and the representation of techni-quarks are rather open (as long as the one-loop beta function coefficient is negative and small), since these particles would be confined under the energy scale of several hundred $\mathrm{TeV}$ and would escape direct detection. This spurred extensive numerical searches of the conformal window (where $\beta\left(g_{*}\right)=0$ ) and the walking regime (where $\beta(g)<0$ but small) on various lattice settings with choices of colors/flavors/representations. Summaries of recent activities with lattice simulations are found in [10-12]. In an attempt to identify the chirally broken phase below the conformal window for the $\mathrm{SU}(3) N_{F}=4$ and 8 systems, Fodor et al. [13] fitted the Dirac spectra of these gauge theories to the analytic results from the chiral GUE (Dyson index $\beta=2$ ). Subsequently, one of the present author (I.K.) and others tried a similar comparison of the Dirac spectrum of the $\mathrm{SU}(2) N_{F}=8$ system (see e.g. [14-16] for the current situation of this system) to the chiral GSE $(\beta=4)[17]$.

For the above approach of fitting Dirac spectra to the corresponding RMT predictions to be practically useful, it is highly desirable to single out individual distributions of each of the ordered RM eigenvalues $p_{k}(x)$ from the spectral density $\rho(x ;\{\mu\})=\left\langle\sum_{k} \delta\left(x-x_{k}\right)\right\rangle=$ $\sum_{k \geq 1} p_{k}(x ;\{\mu\})$, as the latter becomes rather structureless after a couple of oscillations (figure 1). 


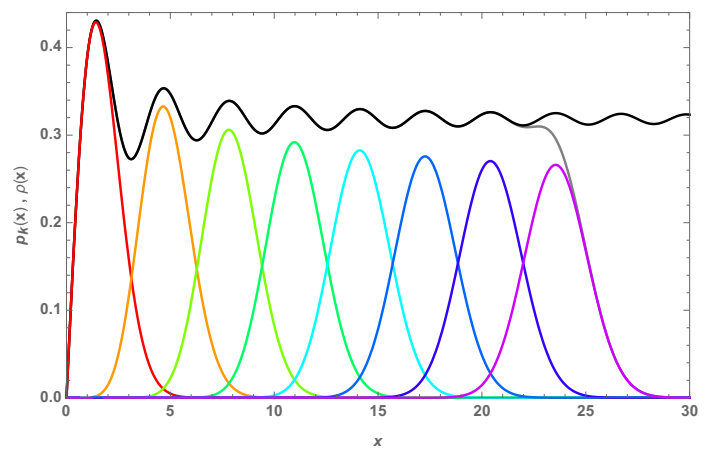

Figure 1. First eight eigenvalue distributions $p_{1}(x), \ldots, p_{8}(x)$ (red to purple), their sum (gray), and the microscopic spectral density $\rho(x)$ (black, normalized as $\rho(\infty)=1 / \pi$ ) of the quenched $\left(N_{F}=0\right)$ chiral Gaussian unitary ensemble.

The standard technique to access such individual eigenvalues is to use the spectral kernel. Once the spectral kernel is obtained, one can give an analytic expression of the distribution. Moreover, by combining Nyström-type (quadrature) evaluation of Fredholm determinants and Pfaffians, one can numerically evaluate the distribution of individual eigenvalues. Damgaard and one of the authors (S.M.N.) have previously derived analytic expressions of such individual eigenvalue distributions for chiral RM ensembles at three Dyson indices $\beta$ and with scaled quark mass parameters $\left\{\mu_{f}\right\}$, initially by the shift-ofvariable method $[18,19]$ and later by the Nyström-type evaluation of Fredholm determinants and Pfaffians of the spectral kernels [20]. There, technical difficulties have prevented us from obtaining analytic formulas for the chiral GSE $(\beta=4)$ with even numbers of massless flavors and for the chiral GOE $(\beta=1)$ with even values of the topological charge. Especially, the former restriction is frustrating, as it obstructs applications to the $\mathrm{SU}(2)$ systems with $N_{F}=8$ and 12 staggered flavors that are popular lattice settings of walking technicolor candidates. Because of this reason, the Monte Carlo method with finite-size matrices was used in [17] to generate the spectral distribution of the RM side in their analysis of $\mathrm{SU}(2) N_{F}=8$ system. The purpose of this paper is to lift this restriction by providing an analytic formula for the conditional gap probability, a.k.a. the Janossy density, that interpolates the ordinary determinantal or Pfaffian formula for the $k$-point correlation function and the Fredholm determinant/Pfaffian expression for the gap probability. Then our formula is numerically evaluated very efficiently by the Nyström-type method. As an application of our result, the low-lying Dirac spectra of the SU(2) lattice gauge theory with $N_{F}=8$ staggered flavors are fitted to the derived RM prediction. ${ }^{1}$

This paper is organized as follows. In section 2 we start by reviewing known formulas on the spectral-statistical distributions of chiral RMTs and their Janossy densities. In

\footnotetext{
${ }^{1}$ We shall use the same data as [17] in this paper, but there are major differences in our analysis from [13]. Our lattice data is obtained with the unimproved staggered fermion action and suffers from large taste breaking effects. Consequently, we do not observe the 4-fold degeneracy characteristic of the staggered tastes, and the lightest of these corresponds to 2 flavors. Moreover, due to the Kramers degeneracy of the $\mathrm{SU}(2)$ Dirac operator, the degeneracy of the lightest fermion modes are 4-fold, to which we must compare the prediction of RMT with $N_{F}=4$ instead of $N_{F}=8$.
} 
section 3 we present a formula for the individual eigenvalue distributions suited for precise numerical evaluation by the Nyström-type method. Specifically, we shall provide numerical data of $p_{1}(x), \ldots, p_{4}(x)$ for the chiral GSE with $N_{F}=4$ and 8 degenerate massive flavors. In section 4 we determine the values of chiral condensate of the SU(2) system with $N_{F}=8$ the first eigenvalue distribution of the corresponding chiral GSE. Conclusions and discussions on feasible applications of our results are presented in section 5. In order to avoid plethora of formulas in RMT and of lattice details in the main text, some of them are relocated to the appendices.

\section{Fredholm determinants and Pfaffians for chiral Gaussian random ma- trix ensembles}

In this section, we will summarize some necessary ingredients about the chiral random matrix ensembles, and derive our main formulae for the Fredholm determinants and Pfaffians of Gaussian chiral random matrix ensembles.

\subsection{Gaussian chiral random matrix ensembles and the microscopic limit}

Consider $N \times(N+\nu)$ matrices $W$ with $W \in \mathbb{R}^{N \times(N+\nu)}, W \in \mathbb{C}^{N \times(N+\nu)}$, or $W \in \mathbb{H}^{N \times(N+\nu)}$. Each ensemble is labelled by the Dyson index $\beta=1,2,4$, respectively. The non-negative integer $\nu$ denotes the corank of the matrix $H=\left(\begin{array}{cc}0 & W \\ W^{\dagger} & 0\end{array}\right)$ and will correspond to the topological charge when $H$ is interpreted as modelling Dirac operator of a gauge theory [21]. Let $Z_{N, \beta, \nu}\left(\left\{m_{a}\right\}\right)$ be the partition function for the Gaussian chiral random matrix ensembles with $\alpha$ parameters $m_{a}(a=1, \ldots, \alpha)$, which will correspond to quark masses, such that

$$
Z_{N, \beta, \nu}\left(\left\{m_{a}\right\}\right)=\int d W \mathrm{e}^{-\beta \operatorname{tr}\left(W^{\dagger} W\right)} \prod_{a=1}^{\alpha} \operatorname{det}\left(\begin{array}{cc}
m_{a} & \mathrm{i} W \\
\mathrm{i} W^{\dagger} & m_{a}
\end{array}\right),
$$

where det stands for the determinant for $\beta=1,2$ and the quaternionic determinant (qdet) for $\beta=4$. In particular for $\beta=4$ it is understood that twofold degenerated eigenvalues in the determinant are only counted once. In terms of eigenvalues $\left\{x_{i}\right\}$ for the Wishart matrix $W^{\dagger} W$, i.e. the squares of non-zero eigenvalues $\left\{ \pm \lambda_{i}\right\}$ of the Hermitian matrix $H$, $Z_{N, \beta, \nu}\left(\left\{m_{a}\right\}\right)$ is expressed as follows:

$$
\begin{aligned}
Z_{N, \beta, \nu}\left(\left\{m_{a}\right\}\right)= & \left(\prod_{a=1}^{\alpha} m_{a}^{\nu}\right) \int_{0}^{\infty} \cdots \int_{0}^{\infty} \prod_{i=1}^{N}\left(d x_{i} x_{i}^{\frac{\beta(\nu+1)}{2}-1} \mathrm{e}^{-\beta x_{i}} \prod_{a=1}^{\alpha}\left(x_{i}+m_{a}^{2}\right)\right) \\
& \times \prod_{i>j}^{N}\left|x_{i}-x_{j}\right|^{\beta} .
\end{aligned}
$$

Likewise the $p$-level correlation function $R_{N, \beta, \nu}^{(p)}\left(\lambda_{1}, \ldots, \lambda_{p} ;\left\{m_{a}\right\}\right)$ of the Hermitian matrix $H$ is defined by

$$
\begin{aligned}
R_{N, \beta, \nu}^{(p)}( & \left.\lambda_{1}, \ldots, \lambda_{p} ;\left\{m_{a}\right\}\right) \\
& =\left(2^{p} \prod_{j=1}^{p}\left|\lambda_{j}\right|\right) \sigma_{N, \beta, \nu}^{(p)}\left(\lambda_{1}^{2}, \ldots, \lambda_{p}^{2} ;\left\{m_{a}\right\}\right),
\end{aligned}
$$




$$
\begin{aligned}
& \sigma_{N, \beta, \nu}^{(p)}\left(x_{1}, \ldots, x_{p} ;\left\{m_{a}\right\}\right) \\
& \quad=\frac{\Sigma_{N, \beta, \nu}^{(p)}\left(x_{1}, \ldots, x_{p} ;\left\{m_{a}\right\}\right)}{\Sigma_{N, \beta, \nu}^{(0)}\left(\left\{m_{a}\right\}\right)}, \\
& \Sigma_{N, \beta, \nu}^{(p)}\left(x_{1}, \ldots, x_{p} ;\left\{m_{a}\right\}\right) \\
& \quad=\frac{1}{(N-p) !} \int_{0}^{\infty} d x_{p+1} \cdots \int_{0}^{\infty} d x_{N} \prod_{i=1}^{N}\left(x_{i}^{\frac{\beta(\nu+1)}{2}-1} \mathrm{e}^{-\beta x_{i}} \prod_{a=1}^{\alpha}\left(x_{i}+m_{a}^{2}\right)\right) \prod_{i>j}^{N}\left|x_{i}-x_{j}\right|^{\beta} .
\end{aligned}
$$

Here we introduce variables $z_{j}$ 's such that

$$
z_{j}=\left\{\begin{array}{ll}
-m_{j}^{2}, & j=1, \ldots, \alpha \\
x_{j-\alpha}, & j=\alpha+1, \ldots \alpha+p
\end{array} .\right.
$$

The $p$-level correlation functions for $\beta=2$ are rewritten as the determinant of the spectral kernel $K\left(z_{i}, z_{j}\right)[22-25]$ :

$$
\sigma_{N, \beta=2, \nu}^{(p)}\left(x_{1}, \ldots, x_{p} ;\left\{m_{a}\right\}\right)=\frac{1}{N ! \Sigma_{N, \beta=2, \nu}^{(0)}\left(\left\{m_{a}\right\}\right)} \operatorname{det}\left[K\left(z_{i}, z_{j}\right)\right]_{i, j=1}^{p+\alpha} .
$$

$R_{N}^{(p)}\left(x_{1}, \ldots, x_{p-\alpha} ;\left\{m_{a}\right\}\right)$ is given by the determinant of the scalar kernel [26, 27]. For $\beta=1,4$, the skew-orthogonal polynomial method involves the quaternionic determinant qdet [28] of the quaternionic kernel [29-32]. In particular, $p$-level correlation functions are given by $(p+\alpha) \times(p+\alpha)$ quaternionic determinants of the quaternionic kernel, which is rewritten by a $2(p+\alpha) \times 2(p+\alpha)$ Pfaffian of its $\mathbb{C}$-number $2 \times 2$ representative (denoted by the same $K\left(z_{i}, z_{j}\right)$ for notational simplicity),

$$
\begin{aligned}
\sigma_{N, \beta=(1,4), \nu}^{(p)}\left(x_{1}, \ldots, x_{p} ;\left\{m_{a}\right\}\right) & =\frac{1}{N ! \Sigma_{N, \beta=1,4, \nu}^{(0)}\left(\left\{m_{a}\right\}\right)} \operatorname{qdet}\left[K\left(z_{i}, z_{j}\right)\right]_{i, j=1}^{p+\alpha} \\
& =\frac{1}{N ! \Sigma_{N, \beta=1,4, \nu}^{(0)}\left(\left\{m_{a}\right\}\right)} \operatorname{Pf}\left(Z\left[K\left(z_{i}, z_{j}\right)\right]_{i, j=1}^{p+\alpha}\right),
\end{aligned}
$$

where $Z=\mathrm{i} \sigma_{2} \otimes \mathbb{I}_{p+\alpha}$ stands for the skew-unit matrix $Z^{2}=-\mathbb{I}_{2(p+\alpha)}$.

Now we will consider the asymptotic limit:

$$
N \rightarrow \infty, \quad x_{i}, m_{a} \rightarrow 0, \quad \zeta_{i}=\sqrt{8 N x_{i}}, \text { and } \mu_{a}=\sqrt{8 N} m_{a}: \text { fixed }
$$

This limit corresponds the microscopic limit of the QCD-like theory on a box of volume $V$ such that

$$
V \rightarrow \infty, \quad m_{a} \rightarrow 0, \quad \mu_{a}=\Sigma V m_{a}: \text { fixed }
$$

where $\Sigma$ stands for the chiral condensate in the chiral limit. 
In this asymptotic limit (2.9), the scaled $p$-level correlation function $R_{\beta=2, \nu}^{(p)}\left(\zeta_{1}, \ldots, \zeta_{p} ;\left\{\mu_{a}\right\}\right)$ for the chiral GUE with $2 \alpha$ dynamical quarks whose masses are doubly degenerated $\mu_{a}=\mu_{a+\alpha}(a=1, \ldots, \alpha)^{2}$ is found as follows [26, 27, 31-33]:

$$
\begin{aligned}
R_{\beta=2, \nu}^{(p)}\left(\zeta_{1}, \ldots, \zeta_{p} ;\left\{\mu_{a}\right\}\right) & \frac{1}{Z_{\beta=2, \nu}\left(\left\{\mu_{a}\right\}\right)} \operatorname{det}\left(\begin{array}{c}
{\left[K_{--}\left(\mu_{a}, \mu_{b}\right)\right]_{a, b=1, \ldots, \alpha}\left[K_{-+}\left(\zeta_{i}, \mu_{b}\right)\right]_{\substack{i=1, \ldots, p \\
b=1, \ldots, \alpha}}} \\
{\left[K_{+-}\left(\mu_{a}, \zeta_{j}\right)\right]_{\substack{a=1, \ldots, \alpha \\
j=1, \ldots, p}}\left[K_{++}\left(\zeta_{i}, \zeta_{j}\right)\right]_{i, j=1, \ldots, p}}
\end{array}\right), \quad(2.11) \\
K_{++}\left(\zeta, \zeta^{\prime}\right) & =\frac{\sqrt{\zeta \zeta^{\prime}}}{\zeta^{\prime 2}-\zeta^{2}}\left[J_{\nu}(\zeta) \zeta^{\prime} J_{\nu+1}\left(\zeta^{\prime}\right)-J_{\nu}\left(\zeta^{\prime}\right) \zeta J_{\nu+1}(\zeta)\right], \\
K_{++}(\zeta, \zeta) & =\frac{\zeta}{2}\left[J_{\nu}(\zeta)^{2}+J_{\nu+1}(\zeta)^{2}\right], \\
K_{+-}\left(\zeta, \mu^{\prime}\right) & =\frac{-\sqrt{\zeta \mu^{\prime}}}{-\mu^{\prime 2}-\zeta^{2}}\left[J_{\nu}(\zeta)\left(-\mu^{\prime}\right) I_{\nu+1}\left(\mu^{\prime}\right)-I_{\nu}\left(\mu^{\prime}\right) \zeta J_{\nu+1}(\zeta)\right], \\
K_{-+}\left(\mu, \zeta^{\prime}\right) & =\frac{-\sqrt{\mu \zeta^{\prime}}}{\zeta^{\prime 2}+\mu^{2}}\left[I_{\nu}(\mu) \zeta^{\prime} J_{\nu+1}\left(\zeta^{\prime}\right)-J_{\nu}\left(\zeta^{\prime}\right)(-\mu) I_{\nu+1}(\mu)\right], \\
K_{--}\left(\mu, \mu^{\prime}\right) & =\frac{\sqrt{\mu \mu^{\prime}}}{\mu^{\prime 2}-\mu^{2}}\left[I_{\nu}(\mu) \mu^{\prime} I_{\nu+1}\left(\mu^{\prime}\right)-I_{\nu}\left(\mu^{\prime}\right) \mu I_{\nu+1}(\mu)\right], \\
K_{--}(\mu, \mu) & =\frac{\mu}{2}\left[I_{\nu}(\mu)^{2}-I_{\nu+1}(\mu)^{2}\right], \quad Z_{\beta=2, \nu}\left(\mu_{1}, \ldots, \mu_{\alpha}\right)=\operatorname{det}\left(\left[K_{--}\left(\mu_{a}, \mu_{b}\right)\right]_{a, b=1, \ldots, \alpha}\right),
\end{aligned}
$$

where $J_{\nu}(x)$ and $I_{\nu}(x)$ denote the Bessel and the modified Bessel functions, respectively,

$$
I_{\nu}(x)=\mathrm{i}^{-\nu} J_{\nu}(\mathrm{i} x)=\sum_{m=0}^{\infty} \frac{1}{m ! \Gamma(m+\nu+1)}\left(\frac{x}{2}\right)^{2 m+\nu} .
$$

For $\beta=4$, the scaled $p$-level correlation function in the asymptotic limit (2.9) is found for $N_{F}=4 \alpha$ quadruply degenerated flavors $\mu_{a}=\mu_{a+\alpha}=\mu_{a+2 \alpha}=\mu_{a+3 \alpha}(a=1, \ldots, \alpha)$ and $N_{F}=2 \alpha$ doubly degenerated flavors $\mu_{a}=\mu_{a+\alpha}(a=1, \ldots, \alpha)$, manifestly in [30].

$$
R_{\beta=4, \nu}^{(p)}\left(\zeta_{1}, \ldots, \zeta_{p} ;\left\{\mu_{a}\right\}\right)=\frac{1}{Z_{\beta=4, \nu}\left(\left\{\mu_{a}\right\}\right)} \operatorname{Pf}\left[Z K_{i j}\right] .
$$

Explicit expressions of matrix elements of the spectral kernels ${ }^{3} Z K_{i j}$ are summarized in appendix A.

\subsection{Individual eigenvalue distributions}

We now focus on the individual distribution of the $k^{\text {th }}$ smallest eigenvalue for the chiral random matrix ensembles [34]. There are various techniques to analyze the gap probabilities [35, 36] such as linear differential equations [37, 38] or Painlevé transcendental

\footnotetext{
${ }^{2}$ In [26], an alternative representation of the $p$-level correlation function $R^{(p)}\left(\zeta_{1}, \ldots, \zeta_{p} ;\left\{\mu_{a}\right\}\right)$ is also found for general mass parameters. (See eq. (B.5) in appendix B.1.)

${ }^{3}$ An explicit formula for the $p$-level correlation function is known as well for the chiral GOE $(\beta=1)[30]$, but the convergence of the Nyström-type discretization of the Fredholm Pfaffian is not guaranteed due to the discontinuity of $\operatorname{sgn}\left(\zeta-\zeta^{\prime}\right)$ in its kernel elements. To avoid such analytical difficulty, we will focus on the study of the Fredholm Pfaffian for the chiral GSE, and leave discussions of the chiral GOE for the future work.
} 
equations á la Tracy-Widom [39, 40]. An alternative method to find individual distribution of the $k^{\text {th }}$ smallest eigenvalue in the asymptotic limit (2.9) has also been developed in $[19,41]$. (See also [42-47].) The procedure of this method consists of three steps [20]:

1. Relate the joint distribution of the first $k$ eigenvalues to the partition function with $\beta k+\beta(\nu+1) / 2-1$ additional masses and a fixed topological charge $2 / \beta+1$.

2. Replace the partition function by the microscopically-scaled form [29, 30, 33, 48] by taking the asymptotic limit (2.9).

3. Integrate over the scaled variables $\zeta_{i}(i=1, \ldots, k)$ in a cell $0 \leq \zeta_{1} \leq \cdots \leq \zeta_{k-1} \leq \zeta_{k}$.

On actual implementation of the above method, the numerical integration over $k$ scaled variables in the third step becomes resource-consuming. To circumvent such technical issue, we will consider Fredholm determinants and Pfaffians for the chiral random matrix ensembles with $\alpha$ mass parameters as the generating function of the joint distribution of the first $k$ eigenvalues, and utilize the quadrature method [49] to evaluate them numerically [5053]. In this section, we will derive a compact formula ${ }^{4}$ of Fredholm determinants and Pfaffians which will be efficient for numerical computations.

Let $P_{N+\alpha, \beta, \nu}\left(x_{1}, \ldots, x_{N+\alpha}\right)$ be the distribution of the probability for all eigenvalues of the rank $N$ matrix,

$$
P_{N+\alpha, \beta, \nu}\left(x_{1}, \ldots, x_{N+\alpha}\right)=\frac{1}{N ! C_{N, \beta, \nu}^{(0)}\left(\left\{m_{a}\right\}\right)} \prod_{i=1}^{N+\alpha} x_{i}^{\frac{\beta(\nu+1)}{2}-1} \mathrm{e}^{-\beta x_{i}} \prod_{i>j}^{N+\alpha}\left|x_{i}-x_{j}\right|^{\beta} .
$$

The $x_{i}$-independent prefactor $C_{N, \beta, \nu}^{(0)}\left(\left\{m_{a}\right\}\right)$ is defined so that $P_{N+\alpha, \beta, \nu}\left(x_{1}, \ldots, x_{N+\alpha}\right)$ obeys the normalization condition.

$$
\int_{-\infty}^{\infty} \cdots \int_{-\infty}^{\infty} d x_{1} \cdots d x_{N+\alpha} P_{N+\alpha, \beta, \nu}\left(x_{1}, \ldots, x_{N+\alpha}\right) \prod_{a=1}^{\alpha} \chi_{\left\{-m_{a}^{2}\right\}}\left(x_{a}\right) \prod_{i=\alpha+1}^{N+\alpha} \chi_{[0, \infty]}\left(x_{i}\right)=1
$$

where $\chi_{I}(x)$ stands for the characteristic function on $I \subset \mathbb{R}$. If $I$ is a line segment $[a, b]$ $(a<b)$ or a semi-infinite line, the characteristic function is given by

$$
\chi_{I}(x)=\left\{\begin{array}{ll}
1 & (x \in I) \\
0 & (x \notin I)
\end{array} .\right.
$$

If $I$ consists of one point $\{y\}$,

$$
\chi_{\{y\}}(x)=\delta(x-y) .
$$

\footnotetext{
${ }^{4}$ In $[54,55]$, what we call $E\left(k ; I ; m_{a}\right)$ with $k=0, \alpha=1$ for the chiral GUE $(\beta=2)$ has essentially been worked out. We would like to thank P. Forrester for kindly reminding us of their works.
} 
Consider the joint probability $E\left(k ; I ;\left\{m_{a}\right\}\right)$ that one finds exactly $k$ eigenvalues on an interval $I$ along the real axis and $\alpha$ eigenvalues in $\mathbb{R}_{<0}$ such that

$$
\begin{aligned}
E\left(k ; I ;\left\{m_{a}\right\}\right)= & \frac{(N+\alpha) !}{k ! \alpha !(N-k) !} \int_{-\infty}^{\infty} \cdots \int_{-\infty}^{\infty} d x_{1} \cdots d x_{N+\alpha} P_{N+\alpha, \beta, \nu}\left(x_{1}, \ldots, x_{N+\alpha}\right) \\
& \times \prod_{a=1}^{\alpha} \chi_{\left\{-m_{a}^{2}\right\}}\left(x_{a}\right) \prod_{j=\alpha+1}^{\alpha+k} \chi_{I}\left(x_{j}\right) \prod_{l=\alpha+k+1}^{N+\alpha}\left(1-\chi_{I}\left(x_{l}\right)\right) .
\end{aligned}
$$

Such a joint probability $E\left(k ; I ;\left\{m_{a}\right\}\right)$ is known as an analytic continuation of the Janossy density $[24,56,57]$. (See appendix $\mathrm{C}$ for the definition of the Janossy density.) The cumulative distribution $F_{k}(s)$ and the probability distribution $p_{k}(s)$ of the $k^{\text {th }}$ smallest positive eigenvalue are expressed by

$$
F_{k}(s)=1-\sum_{\ell=0}^{k-1} E\left(\ell ;[0, s] ;\left\{m_{a}\right\}\right), \quad p_{k}(s)=\frac{\partial}{\partial s} F_{k}(s) .
$$

In the next subsections, we shall show that the generating function $\tau\left(z ; I ;\left\{m_{a}\right\}\right)$ of the probability $E\left(k ; I ;\left\{m_{a}\right\}\right)$ given by

$$
\begin{aligned}
\tau\left(z ; I ;\left\{m_{a}\right\}\right)= & \sum_{k=0}^{N}(1-z)^{k} E\left(k ; I ;\left\{m_{a}\right\}\right) \\
= & \left\langle\sum_{i=1}^{N+\alpha}\left(\prod_{a=1}^{\alpha} \delta\left(x_{i}+m_{a}^{2}\right) \prod_{j(\neq i)}\left(1-z \chi_{I}\left(x_{j}\right)\right)\right)\right\rangle \\
= & \frac{(N+\alpha) !}{\alpha ! N !} \int_{-\infty}^{\infty} \cdots \int_{-\infty}^{\infty} d x_{1} \cdots d x_{N+\alpha} P_{N+\alpha, \beta, \nu}\left(x_{1}, \ldots, x_{N+\alpha}\right) \\
& \times \prod_{a=1}^{\alpha} \chi_{\left\{-m_{a}^{2}\right\}}\left(x_{a}\right) \prod_{j=\alpha+1}^{N+\alpha}\left(1-z \chi_{I}\left(x_{j}\right)\right)
\end{aligned}
$$

is rewritten as a block-decomposed Fredholm determinant or Pfaffian of the spectral kernels in $(2.7)$ or $(2.8)$.

\subsection{Fredholm determinant for chiral Gaussian unitary ensemble}

We start by sketching the proof for the simplest case $\beta=2, \alpha=1, m_{1}^{2}=-y$ :

$$
\begin{aligned}
& \tau(z ; I ; \sqrt{-y}) \\
&=(N+1)\left(\int-z \int_{I} d x_{2}\right) \cdots\left(\int-z \int_{I} d x_{N+1}\right) P_{N+1, \beta=2, \nu}\left(y, x_{2}, \ldots, x_{N+1}\right) \\
&=(N+1) \int d x_{2} \cdots d x_{N+1} P_{N+1, \beta=2, \nu}\left(y, x_{2}, \cdots, x_{N+1}\right) \\
&-(N+1) N z \int_{I} d x_{2} \int d x_{3} \cdots d x_{N+1} P_{N+1, \beta=2, \nu}\left(y, x_{2}, x_{3}, \cdots, x_{N+1}\right) \\
&+(N+1) \frac{N(N-1)}{2 !} z^{2} \int_{I} d x_{2} d x_{3} \int d x_{4} \cdots d x_{N+1} P_{N+1, \beta=2, \nu}\left(y, x_{2}, x_{3}, x_{4}, \cdots, x_{N+1}\right) \\
&-\cdots
\end{aligned}
$$




$$
\begin{aligned}
= & \sigma_{N, \beta=2, \nu}^{(0)}(\sqrt{-y})-z \int_{I} d x_{2} \sigma_{N, \beta=2, \nu}^{(1)}\left(x_{2} ; \sqrt{-y}\right) \\
& +\frac{z^{2}}{2 !} \int_{I} d x_{2} d x_{3} \sigma_{N, \beta=2, \nu}^{(2)}\left(x_{2}, x_{3} ; \sqrt{-y}\right) \\
& -\frac{z^{3}}{3 !} \int_{I} d x_{2} d x_{3} d x_{4} \sigma_{N, \beta=2, \nu}^{(3)}\left(x_{2}, x_{3}, x_{4} ; \sqrt{-y}\right)+\cdots
\end{aligned}
$$

To rewrite correlation functions $\sigma_{N, \beta=2, \nu}^{(k)}$ in terms of the spectral kernel (2.25), we will prepare some notations such as ${ }^{5}$

$$
(f \circ g)\left(x, x^{\prime}\right)=\int_{I} d x^{\prime \prime} f\left(x, x^{\prime \prime}\right) g\left(x^{\prime \prime}, x^{\prime}\right), \quad \operatorname{tr} f=\int_{I} d x f(x, x), \quad \overbrace{K \circ K \circ \cdots \circ K}^{n}=K^{n} .
$$

In addition, we assume that the quadrature discretization of the Riemann integral on $I$ to be well-defined in the continuum limit $M \rightarrow \infty$ (which is always implicit below),

$$
\left\{x_{1}, \ldots, x_{M}\right\} \in I, \quad d x_{1}, \ldots, d x_{M}>0, \quad \sum_{i=1}^{M} f\left(x_{i}\right) d x_{i} \stackrel{M \rightarrow \infty}{\longrightarrow} \int_{I} f(x) d x .
$$

We further introduce following notations for the block decomposition of the spectral kernel integrated over $I$.

$$
\begin{aligned}
\kappa & =K(-y,-y), & \boldsymbol{k} & =\left[\sqrt{d x_{i}} K\left(x_{i},-y\right)\right]_{i=1, \ldots, M}, \\
\boldsymbol{k}^{\mathrm{T}} & =\left[\sqrt{d x_{i}} K\left(-y, x_{j}\right)\right]_{j=1, \ldots, M}, & \boldsymbol{K} & =\left[\sqrt{d x_{i}} K\left(x_{i}, x_{j}\right) \sqrt{d x_{j}}\right]_{i, j=1, \ldots, M} .
\end{aligned}
$$

Adopting eq. (2.7) and these notations, one can rewrite the Fredholm determinant $\tau(z ; I ; \sqrt{-y})$ in terms of the block-decomposed scalar kernel as follows:

$$
\begin{aligned}
& \tau(z ; I ; \sqrt{-y}) \cdot Z_{N, \beta=2, \nu}(\sqrt{-y}) \\
& =K(-y,-y)-z \int_{I} d x_{2} \operatorname{det}\left|\begin{array}{cc}
K(-y,-y) & K\left(-y, x_{2}\right) \\
K\left(x_{2},-y\right) & K\left(x_{2}, x_{2}\right)
\end{array}\right| \\
& +\frac{z^{2}}{2 !} \int_{I} d x_{2} d x_{3} \operatorname{det}\left|\begin{array}{ccc}
K(-y,-y) & K\left(-y, x_{2}\right) & K\left(-y, x_{3}\right) \\
K\left(x_{2},-y\right) & K\left(x_{2}, x_{2}\right) & K\left(x_{2}, x_{3}\right) \\
K\left(x_{3},-y\right) & K\left(x_{3}, x_{2}\right) & K\left(x_{3}, x_{3}\right)
\end{array}\right| \\
& -\frac{z^{3}}{3 !} \int_{I} d x_{2} d x_{3} d x_{4} \operatorname{det}\left|\begin{array}{llll}
K(-y,-y) & K\left(-y, x_{2}\right) & K\left(y, x_{3}\right) & K\left(-y, x_{4}\right) \\
K\left(x_{2},-y\right) & K\left(x_{2}, x_{2}\right) & K\left(x_{2}, x_{3}\right) & K\left(x_{2}, x_{4}\right) \\
K\left(x_{3},-y\right) & K\left(x_{3}, x_{2}\right) & K\left(x_{3}, x_{3}\right) & K\left(x_{3}, x_{4}\right) \\
K\left(x_{4},-y\right) & K\left(x_{4}, x_{2}\right) & K\left(x_{4}, x_{3}\right) & K\left(x_{4}, x_{4}\right)
\end{array}\right|+\cdots \\
& =\kappa-z\left\{\kappa \operatorname{tr} \boldsymbol{K}-\boldsymbol{k}^{\mathrm{T}} \boldsymbol{k}\right\}+\frac{z^{2}}{2 !}\left\{\kappa(\operatorname{tr} \boldsymbol{K})^{2}-\kappa \operatorname{tr} \boldsymbol{K}^{2}-2 \boldsymbol{k}^{\mathrm{T}} \boldsymbol{k} \operatorname{tr} \boldsymbol{K}+2 \boldsymbol{k}^{\mathrm{T}} \boldsymbol{K} \boldsymbol{k}\right\} \\
& -\frac{z^{3}}{3 !}\left\{\kappa(\operatorname{tr} \boldsymbol{K})^{3}-3 \kappa \operatorname{tr} \boldsymbol{K} \operatorname{tr} \boldsymbol{K}^{2}+2 \kappa \operatorname{tr} \boldsymbol{K}^{3}-3 \boldsymbol{k}^{\mathrm{T}} \boldsymbol{k}(\operatorname{tr} \boldsymbol{K})^{2}+3 \boldsymbol{k}^{\mathrm{T}} \boldsymbol{k} \operatorname{tr} \boldsymbol{K}^{2}\right. \\
& \left.-6 \boldsymbol{k}^{\mathrm{T}} \boldsymbol{K} \boldsymbol{k} \operatorname{tr} \boldsymbol{K}+6 \boldsymbol{k}^{\mathrm{T}} \boldsymbol{K}^{2} \boldsymbol{k}\right\}+\cdots .
\end{aligned}
$$

\footnotetext{
${ }^{5}$ It is noted that $K \circ K=K$ holds on $\mathbb{R}_{+}$, but $K \circ K \neq K$ on the interval $I$.
} 
Reorganizing summations, one finds

$$
\begin{aligned}
\tau(z ; I ; & \sqrt{-y}) \cdot Z_{N, \beta=2, \nu}(\{-y\}) \\
= & \kappa\left\{1-\operatorname{tr} z \boldsymbol{K}+\frac{1}{2 !}(\operatorname{tr} z \boldsymbol{K})^{2}-\frac{1}{3 !}(\operatorname{tr} z \boldsymbol{K})^{3}+\cdots\right\} \\
& \times\left\{1-\frac{1}{2} \operatorname{tr}(z \boldsymbol{K})^{2}+\cdots\right\}\left\{1-\frac{1}{3} \operatorname{tr}(z \boldsymbol{K})^{3}+\cdots\right\} \cdots \\
& +z \boldsymbol{k}^{\mathrm{T}} \boldsymbol{k}\left\{1-\operatorname{tr} z \boldsymbol{K}+\frac{1}{2 !}(\operatorname{tr} z \boldsymbol{K})^{2}-\cdots\right\}\left\{1-\frac{1}{2} \operatorname{tr}(z \boldsymbol{K})^{2}+\cdots\right\} \cdots \\
& +z^{2} \boldsymbol{k}^{\mathrm{T}} \boldsymbol{K} \boldsymbol{k}\{1-\operatorname{tr} z \boldsymbol{K}+\cdots\} \cdots \\
& +z^{3} \boldsymbol{k}^{\mathrm{T}} \boldsymbol{K}^{2} \boldsymbol{k}\{1-\cdots\} \cdots \\
& +\cdots \\
= & \left\{\kappa+z \boldsymbol{k}^{\mathrm{T}}\left(\mathbb{I}+z \boldsymbol{K}+(z \boldsymbol{K})^{2}+\cdots\right) \boldsymbol{k}\right\} \\
& \times \exp \left(-\operatorname{tr} z \boldsymbol{K}-\frac{1}{2} \operatorname{tr}(z \boldsymbol{K})^{2}-\frac{1}{3} \operatorname{tr}(z \boldsymbol{K})^{3}-\frac{1}{4} \operatorname{tr}(z \boldsymbol{K})^{4}-\cdots\right) \\
= & \left\{\kappa+z \boldsymbol{k}^{\mathrm{T}}(\mathbb{I}-z \boldsymbol{K})^{-1} \boldsymbol{k}\right\} \operatorname{det}(\mathbb{I}-z \boldsymbol{K})=-\operatorname{det}|-\kappa-\sqrt{z} \boldsymbol{k} \mathbb{I}-z \boldsymbol{K}|
\end{aligned}
$$

Thus we obtain a compact expression of $\tau(z ; I ; \sqrt{-y})$ in terms of the Fredholm determinant.

The generalization to the case with $\alpha$ eigenvalues lying at $y_{a}(a=1, \ldots, \alpha)$ proceeds in the same way as the derivation of eq. (2.26), leading to

$$
\tau\left(z ; I ;\left\{\sqrt{-y_{q}}\right\}\right)=\frac{\operatorname{det}\left|\begin{array}{cc}
-\kappa & -\sqrt{z} \boldsymbol{k}^{\mathrm{T}} \\
-\sqrt{z} \boldsymbol{k} \mathbb{I}-z \boldsymbol{K}
\end{array}\right|}{\operatorname{det}(-\kappa)}:=\frac{\operatorname{det} \mathcal{K}(z)}{\operatorname{det}(-\kappa)},
$$

where the notation for the block decomposition of kernels (2.25) is generalized as

$$
\begin{aligned}
& \kappa=\left[K\left(-y_{a},-y_{b}\right)\right]_{a, b=1, \ldots, \alpha}, \quad \quad k=\left[\sqrt{d x_{i}} K\left(x_{i},-y_{b}\right)\right]_{\substack{i=1, \ldots, M \\
b=1, \ldots, \alpha}}, \\
& \boldsymbol{k}^{\mathrm{T}}=\left[K\left(-y_{a}, x_{j}\right) \sqrt{d x_{j}}\right]_{\substack{a=1, \ldots, \alpha \\
j=1, \ldots, M}}, \quad \boldsymbol{K}=\left[\sqrt{d x_{i}} K\left(x_{i}, x_{j}\right) \sqrt{d x_{j}}\right]_{i, j=1, \ldots, M} .
\end{aligned}
$$

The numerator $\mathcal{K}(z)$ clearly interpolates the (ordinary) determinantal form for the $k$-level correlation function $\operatorname{det} \kappa$ in the case $I \rightarrow \emptyset$ (for which $\boldsymbol{k}, \boldsymbol{K} \rightarrow 0$ ) and the Fredholm determinantal form $\operatorname{det}(\mathbb{I}-z \boldsymbol{K})$ for the generating function of the gap probability in the 'quenched' limit $y_{a} \rightarrow \infty$ (for which $\kappa \rightarrow \mathbb{I}$ and $\boldsymbol{k} \rightarrow 0$ ).

For $y_{a}>0$ and $y_{a} \in I, \tau\left(1 ; I ;\left\{\sqrt{-y_{a}}\right\}\right)$ represents the Janossy density $J_{\alpha, I}\left(\left\{y_{a}\right\}\right)$ defined as the probability of finding no eigenvalue in the interval $I$ except for the ones at designated points $y_{a} \in I(a=1, \ldots, \alpha)$, for the (classical) Laguerre unitary ensemble. On the other hand, after an analytic continuation to $y_{a}=-m_{a}^{2}<0$ and setting $I=[0, s]$, 
$\tau\left(1 ; I ;\left\{m_{a}\right\}\right)$ represents the probability $E\left(0 ;[0, s] ;\left\{m_{a}\right\}\right)$ of finding no eigenvalue smaller than $s$ for the massive Laguerre unitary ensemble (see discussions in appendix C.2).

Finally, changing the eigenvalue variables back to the chiral Gaussian and taking the asymptotic limit (2.9), eq. (2.27) leads to

$$
\tau\left(z ; I ;\left\{\mu_{a}\right\}\right)=\frac{\operatorname{det}\left|\begin{array}{cc}
-\kappa & -\sqrt{z} \boldsymbol{k}^{\mathrm{T}} \\
-\sqrt{z} \boldsymbol{k} \mathbb{I}-z \boldsymbol{K}
\end{array}\right|}{\operatorname{det}(-\kappa)}
$$

with the kernel elements given by their scaled forms (2.12),

$$
\begin{aligned}
\kappa & =\left[K_{--}\left(\mu_{a}, \mu_{b}\right)\right]_{a, b=1, \cdots, \alpha}, & \boldsymbol{k} & =\left[\sqrt{d \zeta_{i}} K_{+-}\left(\zeta_{i}, \mu_{b}\right)\right]_{\substack{i=1, \ldots, M \\
b=1, \ldots, \alpha}}, \\
\boldsymbol{k}^{\mathrm{T}} & =\left[\sqrt{d \zeta_{i}} K_{-+}\left(\mu_{a}, \zeta_{j}\right)\right]_{\substack{a=1, \ldots, \alpha \\
j=1, \ldots, M}}, & \boldsymbol{K} & =\left[\sqrt{d \zeta_{i}} K_{++}\left(\zeta_{i}, \zeta_{j}\right) \sqrt{d \zeta_{j}}\right]_{i, j=1, \ldots, M}
\end{aligned}
$$

\subsection{Fredholm Pfaffian for chiral Gaussian symplectic ensemble}

Generalization of the result of the previous subsection to the chiral GOE and GSE is straightforward: one finds the quaternionic determinant formula simply by replacing $K$ with the quaternionic kernel and "det" with "qdet" simultaneously, because the quaternionic determinant shares the same linear algebraic properties which are utilized in the derivation of the determinant formula (2.29). In particular for the chiral GSE, one can use the explicit formulae of the correlation functions and spectral kernels for $N_{F}=4 \alpha$ and $N_{F}=2 \alpha$ in [30]. (See appendix A.) Indeed, applying the correlation functions $R^{(p)}$ in appendix A to eq. (2.22) and repeating the same steps leading to zeq. (2.29), one finds the following Pfaffian formula

$$
\begin{aligned}
\tau\left(z ; I ;\left\{\mu_{a}\right\}\right) & =\frac{\operatorname{qdet}\left|\begin{array}{cc}
-\kappa & -\sqrt{z} \boldsymbol{k}^{\mathrm{T}} \\
-\sqrt{z} \boldsymbol{k} \mathbb{I}-z \boldsymbol{K}
\end{array}\right|}{\operatorname{qdet}(-\kappa)}=\frac{\operatorname{Pf}\left[Z \mathbb{J}_{2 \alpha}-Z(z \circ K)\right]}{\operatorname{Pf}\left[-Z K^{(0)}\right]} \\
& =\frac{\sqrt{\operatorname{det}\left[\mathbb{J}_{2 \alpha}-z \circ K\right]}}{\sqrt{\operatorname{det} K^{(0)}}}:=\frac{\sqrt{\operatorname{det} \mathcal{K}(z)}}{\sqrt{\operatorname{det} K^{(0)}}},
\end{aligned}
$$

where

$$
\mathbb{J}_{2 \alpha}=\operatorname{diag}(\overbrace{0, \cdots, 0}^{2 \alpha}, 1,1, \cdots)
$$

The matrix elements $S_{A B}, D_{A B}$, and $I_{A B}(A, B= \pm)$ of the quaternionic kernel $K$ are given in eqs. (A.2)-(A.4).

For the quadruply degenerated case $N_{F}=4 \alpha, z \circ K$ with $\mu_{a}(a=1, \ldots, \alpha)$ is given by

$$
z \circ K=\left(\begin{array}{cc}
{\left[K_{--}\left(\mu_{a}, \mu_{b}\right)\right]} & \sqrt{z}\left[K_{+-}\left(\mu_{a}, \zeta_{j}\right) \sqrt{d \zeta_{j}}\right] \\
\sqrt{z}\left[\sqrt{d \zeta_{i}} K_{-+}\left(\zeta_{i}, \mu_{b}\right)\right] & z\left[\sqrt{d \zeta_{i}} K_{++}\left(\zeta_{i}, \zeta_{j}\right) \sqrt{d \zeta_{j}}\right]
\end{array}\right),
$$


where $Z=\mathrm{i} \sigma_{2} \otimes \mathbb{I}_{\alpha+M}$, and

$$
\begin{aligned}
& K_{A B}=\left(\begin{array}{c}
{\left[-S_{A B}\left(\xi_{A}, \xi_{B}\right)\right]\left[-I_{A B}\left(\xi_{A}, \xi_{B}\right)\right]} \\
{\left[D_{A B}\left(\xi_{A}, \xi_{B}\right)\right]\left[-S_{A B}^{\mathrm{T}}\left(\xi_{A}, \xi_{B}\right)\right]}
\end{array}\right), \\
& \left(\xi_{+}, d \xi_{+}\right)=(\zeta, d \zeta), \quad\left(\xi_{-}, d \xi_{-}\right)=(\mu, 1) \text {. }
\end{aligned}
$$

For the doubly degenerated case $N_{F}=2 \alpha, z \circ K$ for even $\alpha$ with $y_{a}=-\mu_{a}^{2}(a=1, \ldots, \alpha)$ is given by

$$
Z(z \circ K)=\left(\begin{array}{ccc}
{\left[I_{--}\left(\mu_{a}, \mu_{b}\right)\right]} & \sqrt{z}\left[I_{-+}\left(\mu_{a}, \zeta_{j}\right) \sqrt{d \zeta_{j}}\right] & \sqrt{z}\left[S_{-+}\left(\mu_{a}, \zeta_{j}\right) \sqrt{d \zeta_{j}}\right] \\
-\sqrt{z}\left[\sqrt{d \zeta_{i}} I_{-+}^{\mathrm{T}}\left(\mu_{b}, \zeta_{i}\right)\right] & z\left[\sqrt{d \zeta_{i}} I_{++}\left(\zeta_{i}, \zeta_{j}\right) \sqrt{d \zeta_{j}}\right] & z\left[\sqrt{d \zeta_{i}} S_{++}\left(\zeta_{i}, \zeta_{j}\right) \sqrt{d \zeta_{j}}\right] \\
-\sqrt{z}\left[\sqrt{d \zeta_{i}} S_{-+}^{\mathrm{T}}\left(\mu_{b}, \zeta_{i}\right)\right] & -z\left[\sqrt{d \zeta_{i}} S_{++}^{\mathrm{T}}\left(\zeta_{j}, \zeta_{i}\right) \sqrt{d \zeta_{j}}\right] & z\left[\sqrt{d \zeta_{j}} D_{++}\left(\zeta_{i}, \zeta_{j}\right) \sqrt{d \zeta_{i}}\right]
\end{array}\right),
$$

and $z \circ K$ for odd $\alpha$ is by

$$
\begin{aligned}
& Z(z \circ K) \\
& \quad=\left(\begin{array}{cccc}
{\left[I_{--}\left(\mu_{a}, \mu_{b}\right)\right]} & {\left[Q_{-}\left(\mu_{a}\right)\right]} & \sqrt{z}\left[I_{-+}\left(\mu_{a}, \zeta_{j}\right) \sqrt{d \zeta_{j}}\right] & \sqrt{z}\left[S_{-+}\left(\mu_{a}, \zeta_{j}\right) \sqrt{d \zeta_{j}}\right] \\
-\left[Q_{-}^{\mathrm{T}}\left(\mu_{b}\right)\right] & 0 & -\sqrt{z}\left[Q_{+}^{\mathrm{T}}\left(\zeta_{j}\right) \sqrt{d \zeta_{j}}\right] & -\sqrt{z}\left[P_{+}^{\mathrm{T}}\left(\zeta_{j}\right) \sqrt{d \zeta_{j}}\right] \\
-\sqrt{z}\left[\sqrt{d \zeta_{i}} I_{-+}^{\mathrm{T}}\left(\mu_{b}, \zeta_{i}\right)\right] & \sqrt{z}\left[\sqrt{d \zeta_{i}} Q_{+}\left(\zeta_{i}\right)\right] & z\left[\sqrt{d \zeta_{i}} I_{++}\left(\zeta_{i}, \zeta_{j}\right) \sqrt{d \zeta_{j}}\right] & z\left[\sqrt{d \zeta_{i}} S_{++}\left(\zeta_{i}, \zeta_{j}\right) \sqrt{d \zeta_{j}}\right] \\
-\sqrt{z}\left[\sqrt{d \zeta_{i}} S_{-+}^{\mathrm{T}}\left(\mu_{b}, \zeta_{i}\right)\right] & \sqrt{z}\left[\sqrt{d \zeta_{i}} P_{+}\left(\zeta_{i}\right)\right] & -z\left[\sqrt{d \zeta_{i}} S_{++}^{\mathrm{T}}\left(\zeta_{j}, \zeta_{i}\right) \sqrt{d \zeta_{j}}\right] & z\left[\sqrt{d \zeta_{i}} D_{++}\left(\zeta_{i}, \zeta_{j}\right) \sqrt{d \zeta_{j}}\right]
\end{array}\right) .
\end{aligned}
$$

Matrix elements of $z \circ K$ in the asymptotic limit (2.9) are summarized in eqs. (A.2)-(A.4), and (A.8)-(A.9).

In case that some of the masses $\mu_{a}$ 's are degenerated, one should adopt the confluent limit of the spectral kernel. Some details of the confluent limit of the spectral kernel is discussed in appendix B.

\section{Numerical evaluation of the Janossy density via the Nyström-type discretization}

In evaluating the Fredholm determinant (2.27) and Pfaffian (2.31) numerically, the Nyström-type discretization proves to be a highly efficient $\operatorname{method}^{6}[58,59]$.This numerical method is based on the quadrature rule (see a brief summary in appendix E), and in seminal works by F. Bornemann, it is shown that the Nyström-type discretization of Fredholm determinants of integral operators of trace class (i.e. for unitary and symplectic ensembles) converges exponentially as the order of the discretization grows. In the following, we employ the Gauss-Legendre quadrature rule of order $M$ with the nodes $\zeta_{i}$ and the weights $w_{i}=d \zeta_{i}(i=1, \ldots, M)$ given in eq. (E.5).

\footnotetext{
${ }^{6}$ We will compare our results with the Monte Carlo simulations to examine the efficiency of this method. See appendix F on details of the Monte Carlo simulation.
} 

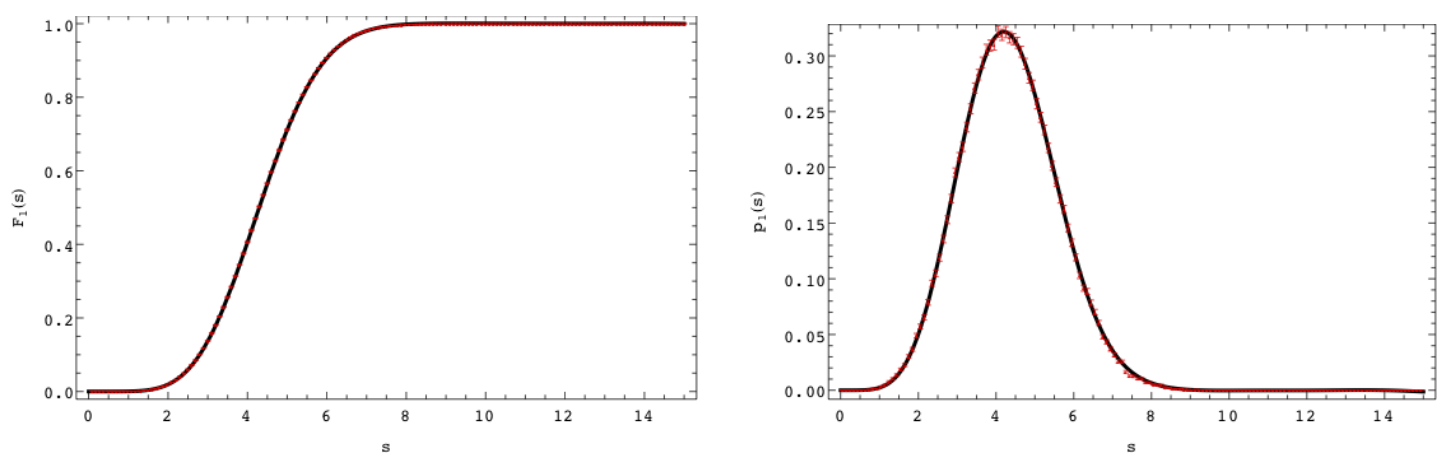

Figure 2. Plots of $F_{1}(s)$ (left) and $p_{1}(s)$ (right) for the chiral GUE with $N_{f}=2$ doubly-degenerated masses $\mu_{1}=0.1$ and the topological charge $\nu=0$ are depicted. In this computation, the quadrature of order $M=5$ is used to discretize the Fredholm determinant (3.1). On both plots, data obtained with Monte Carlo simulation with matrix rank $N=1000$ is overlaid (red symbol with error bar, though the error in the left panel is hard to recognize by eye).

\subsection{Chiral GUE with doubly degenerated masses $N_{F}=2 \alpha$}

The Nyström-type discretization of the Fredholm determinant for the individual eigenvalue distribution with $\beta=2$ and $N_{F}=2 \alpha$ is given as follows:

$$
\begin{aligned}
& \tau\left(z ;[0, s] ;\left\{\mu_{a}\right\}\right) \\
& =\operatorname{det}\left(\begin{array}{cc}
-\left[K_{--}\left(\mu_{a}, \mu_{b}\right)\right]_{a, b=1, \ldots \alpha} & -\sqrt{z}\left[K_{-+}\left(\mu_{a}, \zeta_{j}\right) \sqrt{w_{j}}\right]_{\substack{a=1, \ldots, \alpha \\
j=1, \ldots, M}} \\
-\sqrt{z}\left[\sqrt{w_{i}} K_{+-}\left(\zeta_{i}, \mu_{b}\right)\right]_{\substack{i=1, \ldots, M \\
b=1, \ldots, M}} \mathbb{I}_{M}-z\left[\sqrt{w_{i}} K_{++}\left(\zeta_{i}, \zeta_{j}\right) \sqrt{w_{j}}\right]_{i, j=1, \ldots, M}
\end{array}\right) \\
& / \operatorname{det}\left(-\left[K_{--}\left(\mu_{a}, \mu_{b}\right)\right]_{a, b=1, \ldots \alpha}\right),
\end{aligned}
$$

where the matrix elements $K_{A B}$ are found in eq. (2.12).

We will evaluate $F_{1}(s)=1-\tau\left(1 ;[0, s] ; \mu_{1}\right)$ and $p_{1}(s)=\partial_{s} F_{1}(s)$ using the expression (3.1) and compare with the Monte Carlo simulation. For $\alpha=1$ with $\mu_{1}=0.1$ and the topological charge $\nu=0$, we obtain the numerical plots of $F_{1}(s)$ and $p_{1}(s)$ in figure 2 for the rank $M=5$ of the Gaussian quadrature and find a good agreement with the Monte Carlo simulation with the matrix rank $N=1000$.

\subsection{Chiral GSE with quadruply degenerated masses $N_{F}=4 \alpha$}

For $\beta=4$ and with $N_{F}=4 \alpha$, the Nyström-type discretization of the Fredholm Pfaffian is given by

$$
\begin{aligned}
\tau\left(z ;[0, s] ;\left\{\mu_{a}\right\}\right) & =\frac{\left|\operatorname{det}^{1 / 2}(\mathcal{K}(z))\right|}{\left|\operatorname{det}^{1 / 2}\left(K_{--}\right)\right|}, \\
\mathcal{K}(z) & =\left(\begin{array}{cc}
-\left[K_{--}\left(\mu_{a}, \mu_{b}\right)\right] & -\sqrt{z}\left[K_{+-}\left(\mu_{a}, \zeta_{j}\right) \sqrt{w_{j}}\right] \\
-\sqrt{z}\left[\sqrt{w_{i}} K_{-+}\left(\zeta_{i}, \mu_{b}\right)\right] & \mathbb{I}_{2 M}-z\left[\sqrt{w_{i}} K_{++}\left(\zeta_{i}, \zeta_{j}\right) \sqrt{w_{j}}\right]
\end{array}\right),
\end{aligned}
$$

where the matrix elements $K_{A B}$ are found in eqs. (A.2)-(A.4). 

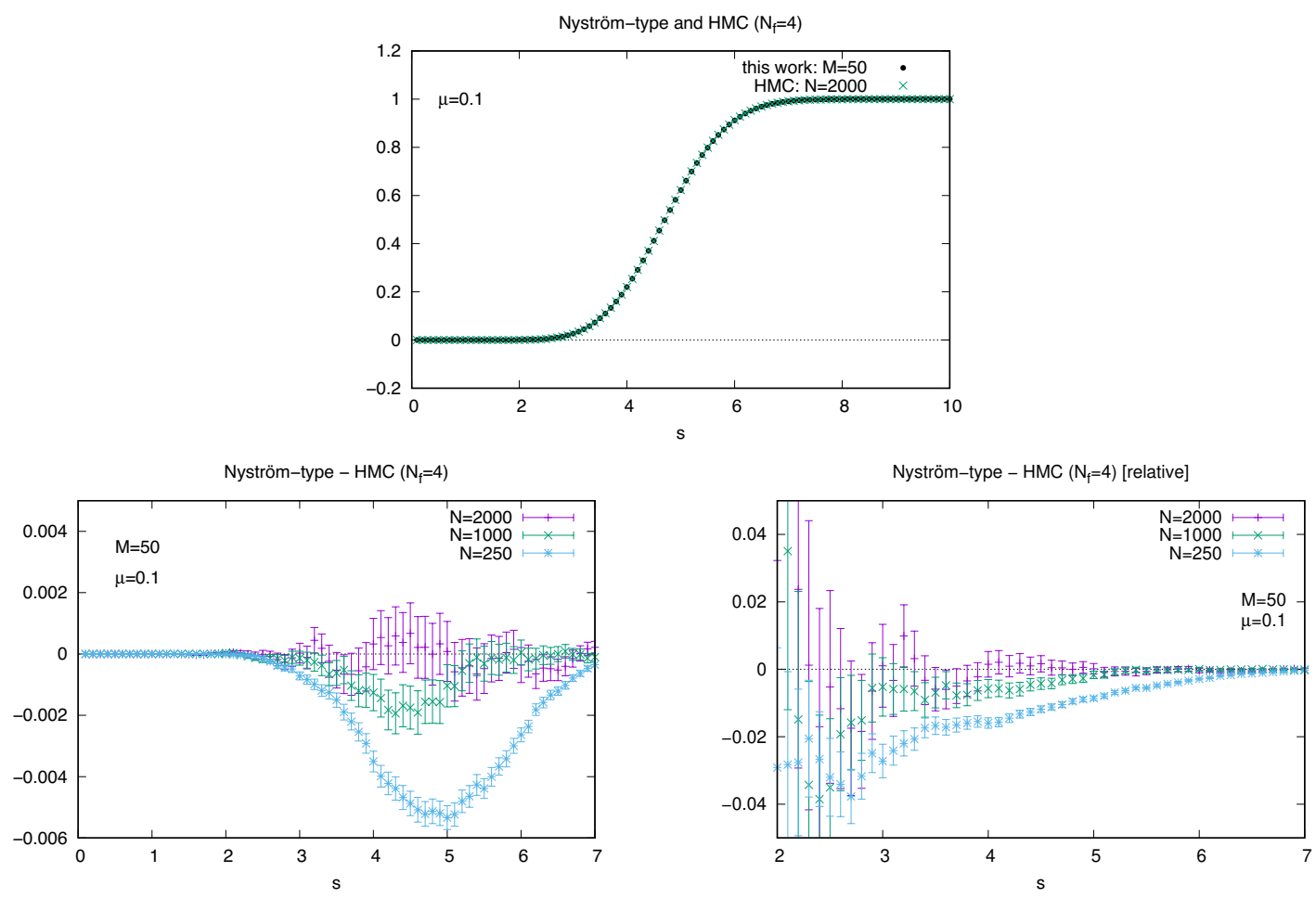

Figure 3. $F_{1}(s)$ is computed for the chiral GSE with $N_{F}=4(\alpha=1)$ quadruply-degenerated masses $\mu_{1}=0.1$ and the topological charge $\nu=0$ in two ways. In the top panel, the Nyström-type discretization of order $M=50$ is applied (black dot) and the hybrid Monte Carlo simulation is applied with the random rank $N=2000, F_{1}^{\mathrm{HMC}}(s)$ (green cross). The error of the HMC result, which is not shown in the top panel, is smaller than the symbols. The bottom left panel shows the difference of these two methods, $F_{1}(s)-F_{1}^{\mathrm{HMC}}(s)$ with $N=250,1000,2000$. The computational results of the hybrid Monte Carlo simulation converges to that of the Nyström-type discretization as $N$ grows. The errors plotted come from the Monte Carlo result. The relative difference normalized by the Nyström-type is also plotted in in the bottom right panel. Note that the relative difference looses its meaning for $s \lesssim 2$ as the Nyström-type result becomes smaller than the Monte Carlo error.

$\boldsymbol{N}_{\boldsymbol{F}}=4$. The numerical plot of $F_{1}(s)$ for $N_{F}=4$ with the quadruply degenerated mass $\mu_{1}=0.1$ and the topological charge $\nu=0$ is depicted in black dots in figure 3 top. In this computation we have chosen $M=50$. In the same figure, the result of the hybrid Monte Carlo simulation $F_{1}^{\mathrm{HMC}}(s)$ of the chiral random matrix ensemble (2.2) with the matrix rank $N=2000$ is shown in green dots as an overlay.

In order to verify the numerical computation with the Nyström-type discretization, we closely looked at the difference $F_{1}(s)-F_{1}^{\mathrm{HMC}}(s)$ for matrix ranks $N=250,1000,2000$. Figure 3 bottom shows that the computational results of the hybrid Monte Carlo simulation indeed converge to the Nyström-type discretization as $N$ grows, confirming that the numerical evaluation of $F_{1}(s)$ by the Nyström-type discretization at $M=50$ is good enough.

The generalized gap probability $E_{k}(s):=E\left(k ;[0, s] ;\left\{\mu_{a}\right\}\right)$ in eq. (2.19) is given by

$$
E_{k}(s)=\left.\frac{(-1)^{k}}{k !} \frac{\partial^{k}}{\partial z^{k}} \tau\left(z ;[0, s] ;\left\{\mu_{a}\right\}\right)\right|_{z=1} .
$$




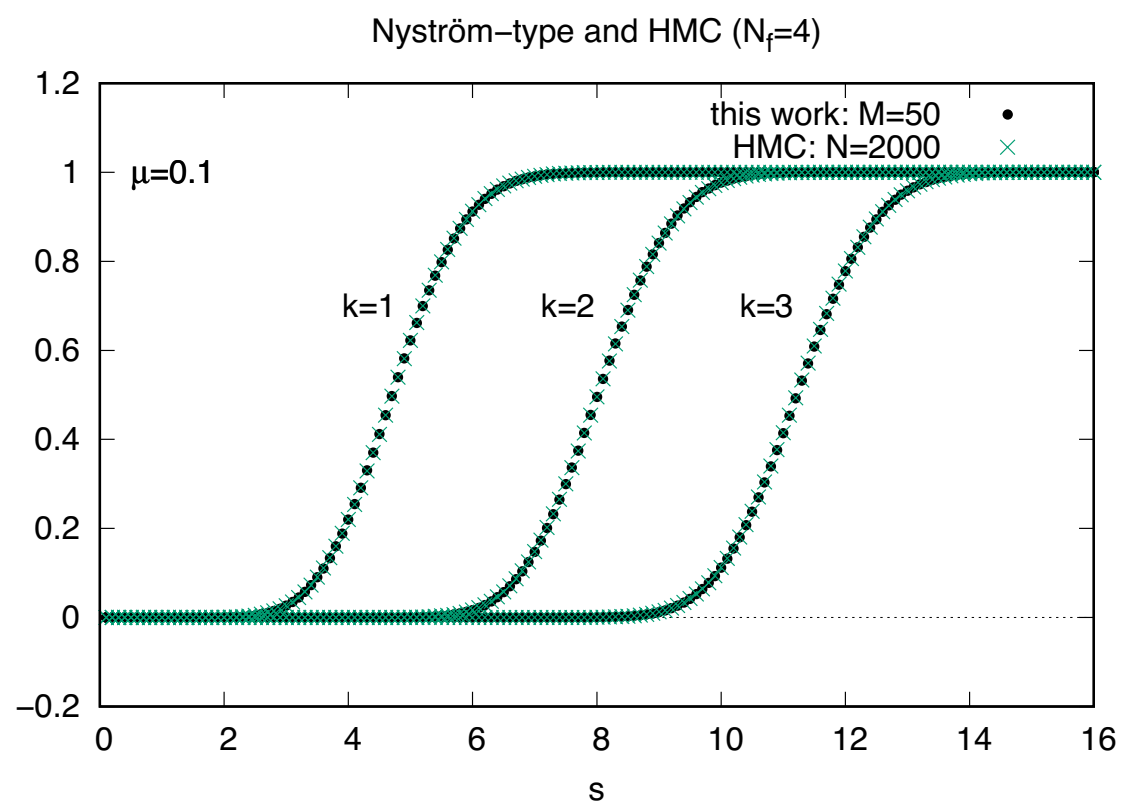

Figure 4. $F_{k}(s)(k=1,2,3)$ for $N_{F}=4$ quadruply-degenerated mass parameters $\mu_{1}=0.1$ and the topological charge $\nu=0$ together with Monte Carlo result. Black dot: Nyström-type discretization of order $M=50$. Green cross: hybrid Monte Carlo simulation with the random matrix rank $N=2000$.

The $z$-derivatives of $\tau\left(z ;[0, s] ;\left\{\mu_{a}\right\}\right)$ are evaluated directly by the Taylor expansion of the determinant, and the explicit expressions of $E_{k}(s)$ 's as the sum of trace factors are listed in appendix D. Numerical computations for $F_{k}(s)$ for $0 \leq s \leq 16$ are depicted in figure 4 , and a good agreement is observed with the computations of the hybrid Monte Carlo simulation with $N=2000$.

$\boldsymbol{N}_{\boldsymbol{F}}=8$. Numerical plots of $F_{k}(s)$ and $p_{k}(s)=\partial_{s} F_{k}(s)$ for $N_{F}=8(\alpha=2)$ with 8-fold degenerated mass $\mu=\mu_{1}=\mu_{2}$ and the topological charge $\nu=0$, computed at $M=128$, are depicted in figures 5 . The computed numerics of $F_{k}(s)$ are appended as Supplementary Material because this case is practically important within our application to the two-color lattice QCD with staggered quarks; the case with $N_{F}=4(\alpha=1)$ doubtlessly has its chiral symmetry broken as in the ordinary QCD, and those with $N_{f} \geq 12(\alpha \geq 3)$ have negative 1-loop $\beta$-function coefficients $\beta_{0}=\left(11 N_{C}-2 N_{F}\right) / 3$ and are IR free. Accordingly, $N_{F}=8$ is the only case which evokes the question of whether its nature is either QCD-like, conformal, or walking (which would nominate the model as a possible candidate for the technicolor scenario), and motivates us to compare its Dirac spectrum to the massive chiral GSE prediction so as to confirm or exclude if it is QCD-like.

\subsection{Chiral GSE with doubly degenerated masses $N_{F}=2 \alpha$}

For $\beta=4$ and with $N_{F}=2 \alpha$, the quaternionic kernel for Janossy density of the $\beta=4$ ensemble is treated independently for even and odd $\alpha$. 

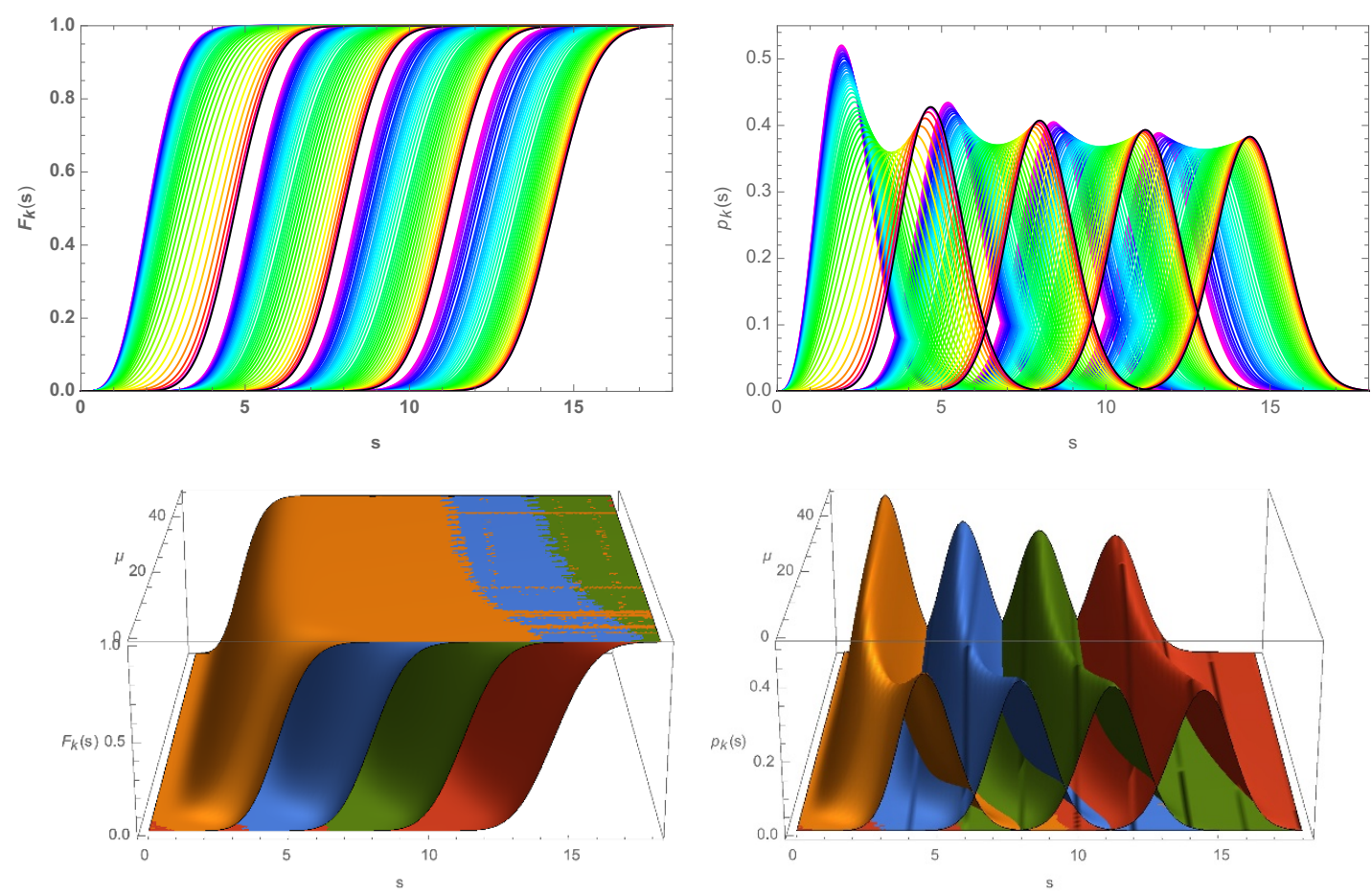

Figure 5. Scaled mass parameter $(\mu)$ dependence of [left panels] cumulative distributions $F_{k}(s)$ and [right panels] probability distributions $p_{k}(s)$ of the four smallest unfolded eigenvalues $k=1,2,3,4$ of the massive chiral GSE with $N_{F}=8$ degenerate flavors and the topological charge $\nu=0$, computed at $M=128$. In the upper panels, mass parameters are chosen at $\mu=0$ (black, $N_{F}=0$ with

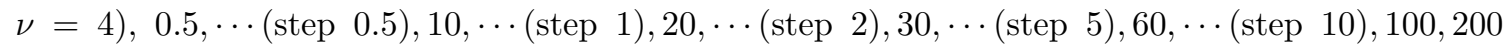
(red to purple), $\infty$ (gray, $N_{F}=0$ with $\nu=0$ ). The lower panels are interpolations of the upper ones.

$\boldsymbol{N}_{\boldsymbol{F}}=\mathbf{2}$. For the case of odd $\alpha$, the Nyström-type discretization of $\tau\left(z ;[0, s] ;\left\{\mu_{a}\right\}\right)$ yields

$$
\begin{aligned}
& \tau\left(z ;[0, s] ;\left\{\mu_{a}\right\}\right) \\
& =\frac{\left|\operatorname{det}^{1 / 2}(\mathcal{K}(z))\right|}{\left|\operatorname{det}^{1 / 2}\left(\mathcal{K}^{(0)}\right)\right|}, \\
& \mathcal{K}(z)=\left(\begin{array}{cccc}
\mathbb{I}_{M}-z\left[\sqrt{w_{i} w_{j}} S_{++}\left(x_{i}, x_{j}\right)\right] & -z\left[\sqrt{w_{i} w_{j}} I_{++}\left(x_{i}, x_{j}\right)\right] & \sqrt{z}\left[\sqrt{w_{j}} Q_{+}\left(x_{j}\right)\right] & \sqrt{z}\left[I_{-+}\left(\mu_{a}, x_{j}\right)\right] \\
z\left[\sqrt{w_{i} w_{j}} D_{++}\left(x_{i}, x_{j}\right)\right] & \mathbb{I}_{M}-z\left[\sqrt{w_{i} w_{j}} S_{++}^{\mathrm{T}}\left(x_{j}, x_{i}\right)\right] & \sqrt{z}\left[\sqrt{w_{j}} P_{+}\left(x_{j}\right)\right] & \sqrt{z}\left[\sqrt{w_{j}} S_{-+}\left(\mu_{a}, x_{j}\right)\right] \\
-\sqrt{z}\left[\sqrt{w_{i}} S_{-+}^{\mathrm{T}}\left(\mu_{b}, x_{i}\right)\right] & -\sqrt{z}\left[\sqrt{w_{i}} I_{-+}^{\mathrm{T}}\left(\mu_{b}, x_{i}\right)\right] & -\left[Q_{-}\left(\mu_{b}\right)\right] & -\left[I_{--}\left(\mu_{a}, \mu_{b}\right)\right] \\
-\sqrt{z}\left[\sqrt{w_{i}} P_{+}^{\mathrm{T}}\left(x_{i}\right)\right] & -\left[\sqrt{w_{i}} Q_{+}^{\mathrm{T}}\left(x_{i}\right)\right] & 0 & -\left[Q_{-}^{\mathrm{T}}\left(\mu_{a}\right)\right]
\end{array}\right), \\
& \mathcal{K}^{(0)}=\left(\begin{array}{cc}
-\left[Q_{-}\left(\mu_{b}\right)\right] & -\left[I_{--}\left(\mu_{a}, \mu_{b}\right)\right] \\
0 & -\left[Q_{-}^{\mathrm{T}}\left(\mu_{a}\right)\right]
\end{array}\right),
\end{aligned}
$$

where the matrix elements are found in eqs. (A.2)-(A.4) and (A.8)-(A.9).

$F_{1}(s)$ for $\alpha=1$ (i.e. $N_{F}=2$ ) with doubly degenerated mass $\mu_{1}=0.1$ and the topological charge $\nu=0$ is evaluated numerically by the Nyström-type discretization of order $M=50$. Plots are depicted in figure 6 (black dots). The hybrid Monte Carlo simulation 

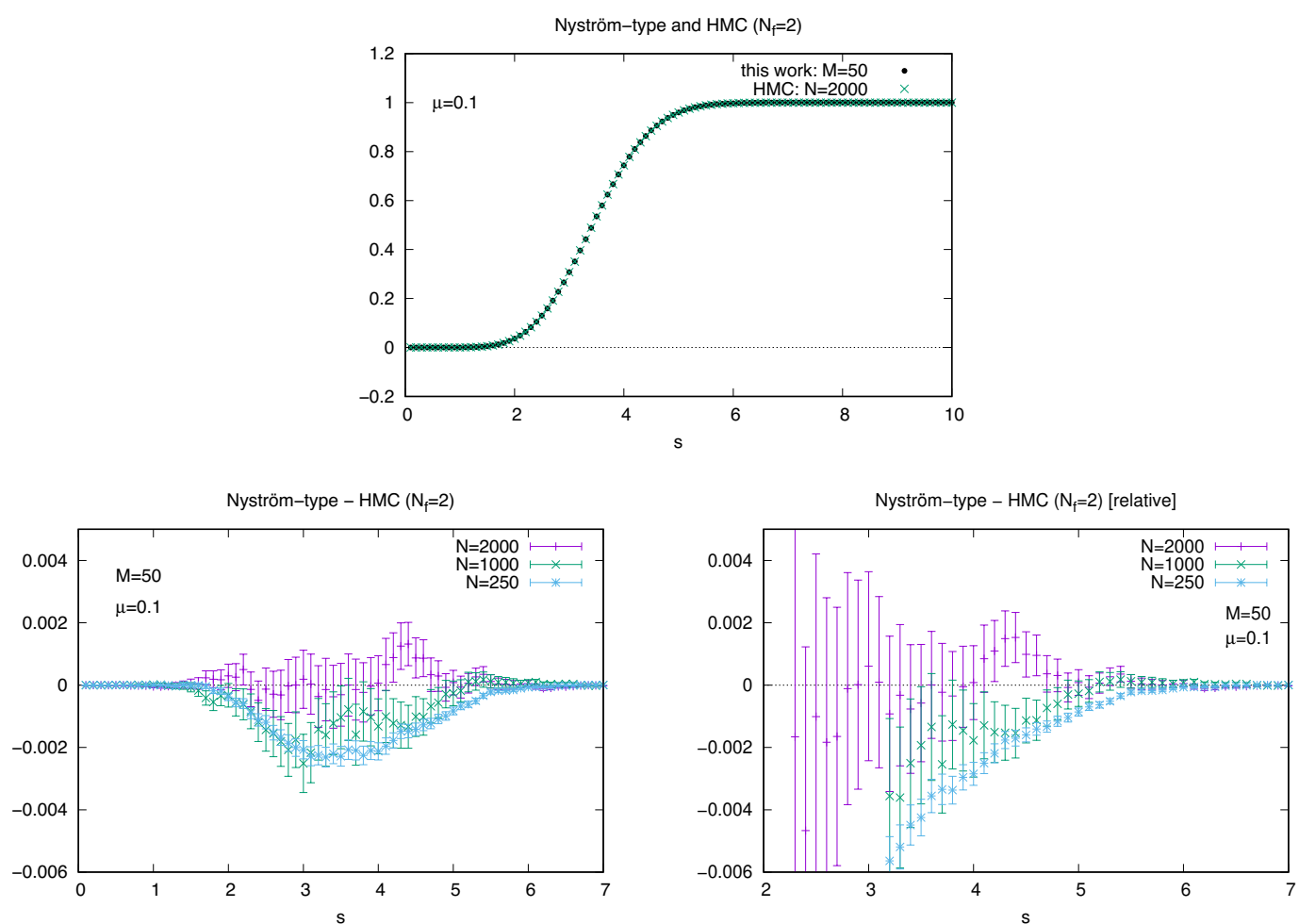

Figure 6. The same plot as figure 3 but with doubly-degenerated masses $\mu_{1}=0.1\left(N_{F}=2\right)$ and the topological charge $\nu=0 . F_{1}(s)$ for the $\beta=4$ ensemble is evaluated in two ways. Top panel: The Nyström-type discretization is applied of order $M=50$ (black dots) and the hybrid Monte Carlo simulation is applied with the random matrix rank $N=2000$ (green cross), for which the statistical errors are smaller than the symbols and not shown in the plot. Bottom panels: Difference between Nyström-type discretization and hybrid Monte Carlo with several values of matrix rank $N$.

$F_{1}^{\mathrm{HMC}}(s)$ of the chiral random matrix ensemble (2.2) with the matrix rank $N=1000$ is depicted (green dots) in figure 6 as an overlay.

The confluent limit for $\boldsymbol{N}_{\boldsymbol{F}}=\mathbf{2}+\mathbf{2}+\mathbf{2}+\mathbf{2}$. The next example is the chiral GSE for $N_{F}=2+2+2+2$ (i.e. $\alpha=4$ ) in the complete confluent limit. The Nyström-type discretization of $E_{0}(s)$ of order $M$ is given by

$$
\begin{aligned}
& E_{0}(s)=\frac{\left|\operatorname{det}^{1 / 2}(\mathcal{K}(z=1))\right|}{\left|\operatorname{det}^{1 / 2} \mathcal{K}^{(0)}\right|}, \quad \mathcal{K}(z=1)=\left(\begin{array}{cc}
\mathcal{S}_{1} & -\mathcal{I} \\
-\mathcal{D} & \mathcal{S}_{2}
\end{array}\right), \\
& \mathcal{S}_{1}=\left(\begin{array}{ccc}
\mathbb{I}_{M}-\left[\sqrt{w_{i} w_{j}} S_{++}\left(\zeta_{i}, \zeta_{j}\right)\right] & {\left[\sqrt{w_{j}} I_{-+}^{(3,0)}\left(\mu, \zeta_{j}\right)\right]} & {\left[\sqrt{w_{j}} I_{-+}^{(2,0)}\left(\mu, \zeta_{j}\right)\right]} \\
-\left[\sqrt{w_{i}} S_{-+}^{(2,0)}(\mu, \zeta)\right] & -I_{--}^{(2,3)}(\mu, \mu) & 0 \\
{\left[\sqrt{w_{i}} S_{-+}^{(3,0)}(\mu, \zeta)\right]} & 0 & -I_{--}^{(2,3)}(\mu, \mu)
\end{array}\right), \\
& \mathcal{I}=\left(\begin{array}{ccc}
{\left[\sqrt{w_{i} w_{j}} I_{++}\left(\zeta_{i}, \zeta_{j}\right)\right]} & -\left[\sqrt{w_{j}} I_{-+}^{(1,0)}\left(\mu, \zeta_{j}\right)\right] & -\left[\sqrt{w_{j}} I_{-+}^{(0,0)}\left(\mu, \zeta_{j}\right)\right] \\
{\left[\sqrt{w_{i}} I_{-+}^{(2,0)}(\mu, \zeta)\right]} & -I_{--}^{(1,2)}(\mu, \mu) & -I_{--}^{(0,2)}(\mu, \mu) \\
-\left[\sqrt{w_{i}} I_{-+}^{(3,0)}(\mu, \zeta)\right] & I_{--}^{(1,3)}(\mu, \mu) & I_{--}^{(0,3)}(\mu, \mu)
\end{array}\right),
\end{aligned}
$$




$$
\begin{aligned}
& \mathcal{D}=\left(\begin{array}{ccc}
-\left[\sqrt{w_{i} w_{j}} D_{++}\left(\zeta_{i}, \zeta_{j}\right)\right] & {\left[\sqrt{w_{j}} S_{-+}^{(1,0)}\left(\mu, \zeta_{j}\right)\right]} & {\left[\sqrt{w_{j}} S_{-+}^{(0,0)}\left(\mu, \zeta_{j}\right)\right]} \\
{\left[\sqrt{w_{i}} S_{-+}^{(0,0)}(\mu, \zeta)\right]} & I_{--}^{(0,3)}(\mu, \mu) & I_{--}^{(0,2)}(\mu, \mu) \\
-\left[\sqrt{w_{i}} S_{-+}^{(1,0)}(\mu, \zeta)\right] & -I_{--}^{(1,3)}(\mu, \mu) & -I_{--}^{(1,2)}(\mu, \mu)
\end{array}\right), \\
& \mathcal{S}_{2}=\left(\begin{array}{ccc}
\mathbb{I}_{M}-\left[\sqrt{w_{i} w_{j}} S_{++}\left(\zeta_{i}, \zeta_{j}\right)\right] & -\left[\sqrt{w_{j}} S_{-+}^{(1,0)}\left(\mu, \zeta_{j}\right)\right] & -\left[\sqrt{w_{j}} S_{-+}^{(0,0)}\left(\mu, \zeta_{j}\right)\right] \\
-\left[\sqrt{w_{i}} I_{-+}^{(0,0)}(\mu, \zeta)\right] & -I_{--}^{(0,1)}(\mu, \mu) & 0 \\
{\left[\sqrt{w_{i}} I_{-+}^{(1,0)}(\mu, \zeta)\right]} & 0 & -I_{--}^{(0,1)}(\mu, \mu)
\end{array}\right) \text {, } \\
& \mathcal{K}^{(0)}=\left(\begin{array}{cccc}
0 & I_{--}^{(0,1)}(\mu, \mu) & I_{--}^{(0,2)}(\mu, \mu) & I_{--}^{(0,3)}(\mu, \mu) \\
-I_{--}^{(0,1)}(\mu, \mu) & 0 & I_{--}^{(1,2)}(\mu, \mu) & I_{--}^{(1,3)}(\mu, \mu) \\
-I_{--}^{(0,2)}(\mu, \mu) & -I_{--}^{(1,2)}(\mu, \mu) & 0 & I_{--}^{(2,3)}(\mu, \mu) \\
-I_{--}^{(0,3)}(\mu, \mu) & -I_{--}^{(1,3)}(\mu, \mu) & -I_{--}^{(2,3)}(\mu, \mu) & 0
\end{array}\right),
\end{aligned}
$$

where the matrix elements $S_{A B}, D_{A B}$, and $I_{A B}(A, B= \pm)$ are found in eqs. (A.2)-(A.4), and $S_{A B}^{(a, b)}, D^{(a, b)_{A B}}$, and $I_{A B}^{(a, b)}(A, B= \pm)$ are found in eqs. (B.15)-(B.17).

For the degenerated mass $\mu=0.1$ and the topological charge $\nu=0$, we find a good agreement of $F_{1}(s)=1-E_{0}(s)$ with the hybrid Monte Carlo simulation $F_{1}^{\mathrm{HMC}}(s)$. The numerical plots are shown in figure 7 (left panel) for $M=50$ of the Nyström discretization of the Fredholm Pfaffian and the hybrid Monte Carlo simulation of the rank $N=2000$. (Black dots: Nyström discretization, Green dots: hybrid Monte Carlo simulation.) The difference $F_{1}(s)-F_{1}^{\mathrm{HMC}}(s)$ in figure 7 (right panel) confirms us that the difference reduces as the rank of matrix grows, and these results confirm us that these numerical computations are consistent and valid.

By applying the explicit expressions in appendix D, we can evaluate $E_{k}(s)$ 's numerically. Plots for $F_{k}(s)$ in $0 \leq s \leq 6$ are depicted in figure 8 , and we find a good agreement with the computations of the hybrid Monte Carlo simulation with $N=4000$.

\section{Application: chiral condensate from lattice data}

As an application of our RMT results, we use the Dirac eigenvalues of the $\mathrm{SU}(2)$ gauge theory with $N_{F}=8$ quarks in the fundamental representation. A partial analysis of this system has been presented in [17], where the Monte Carlo method is used to generate the RMT data. Full analyses using the current RMT result will appear elsewhere [60]. As stated in the Introduction, we should use the chiral GSE with $N_{F}=4$, because due to the taste breaking effect, the 4-fold degeneracy for the staggered fermions is totally broken so the number of lightest flavors is in fact $N_{F}=2$. Furthermore, the pseudo-reality of the $\mathrm{SU}(2)$ gauge group yields an additional 2-fold degeneracy yields an additional 2-fold degeneracy, by which $N_{F}=2$ is promoted to $N_{F}=4$.

The microscopic eigenvalue density is related to the Dirac spectrum through

$$
\zeta_{i}=\lambda_{i} V \Sigma, \quad \quad \mu_{f}=m_{f} V \Sigma,
$$

where $\lambda_{i}$ denotes the eigenvalue of the Dirac operator, $\Sigma$ the chiral condensate, $V$ the 4 -volume, and $m_{f}$ the quark masses. We relate the smallest Dirac eigenvalue distribution 

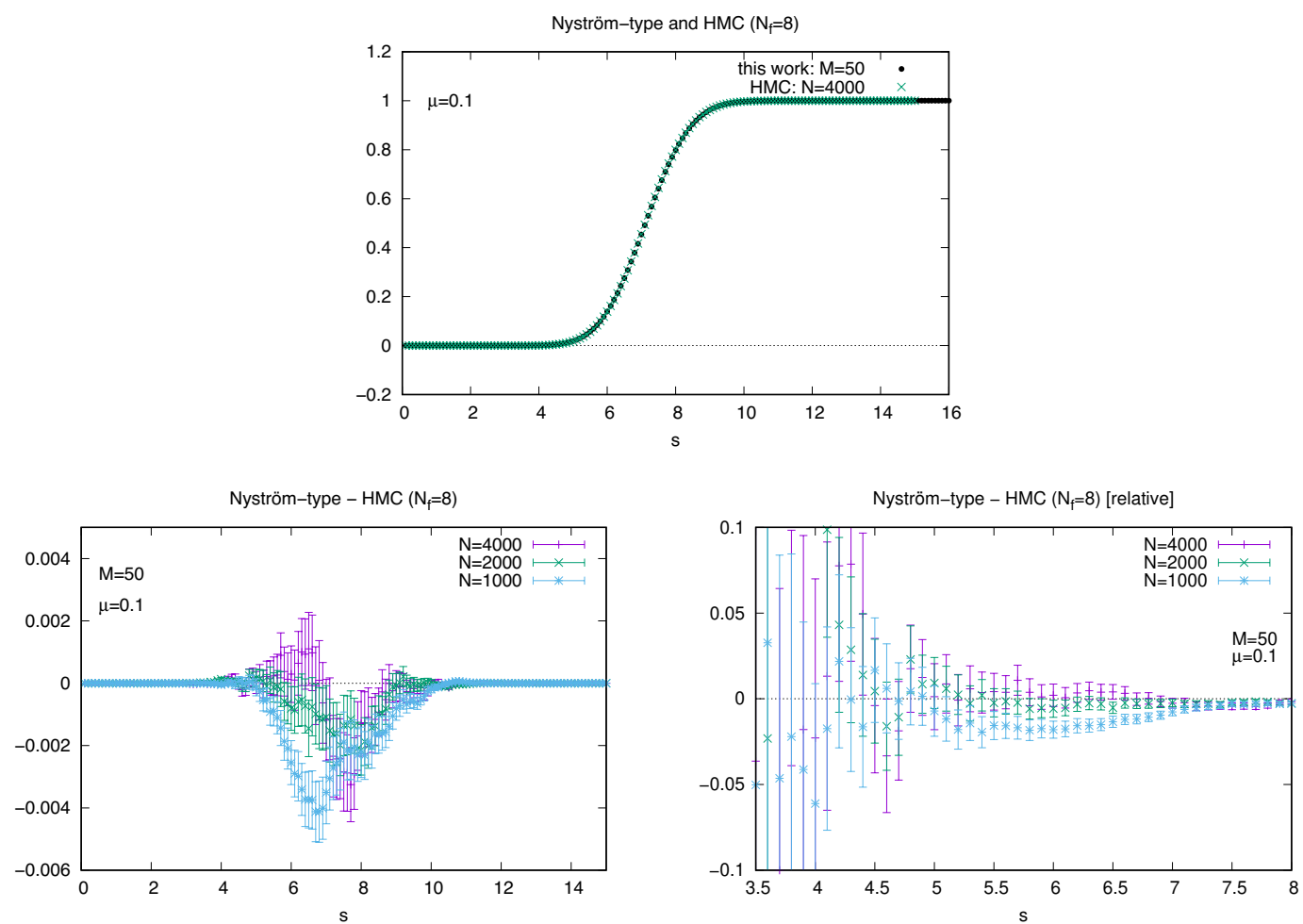

Figure 7. The same plot as figure 3 but with $N_{F}=8 . \quad F_{1}(s)$ is computed in two ways for the chiral GSE with $N_{F}=8$ doubly-degenerated masses in the complete confluent limit with $\mu_{1}=0.1$ and the topological charge $\nu=0$. Nyström-type discretization of order $M=50$ (black dot) and hybrid Monte Carlo simulation with the random rank $N=4000$ are applied (green cross) in the top panel, and the random rank $N=4000$ (green dots in the top figure), respectively. The errors for HMC is smaller than the symbols and not shown in the plot. The bottom panels show the difference $F_{1}(s)-F_{1}^{\mathrm{HMC}}(s)$ for $N=1000,2000,4000$. As the $N$ grows, the HMC results converge to the result from Nyström-type discretization. Compared with the previous cases, however, its convergence is slower.

from lattice simulation through ${ }^{7}$

$$
\left.p_{1}^{\mathrm{RMT}}\left(\zeta_{1} ; \mu\right)\right|_{\zeta_{1}=\lambda_{1} V \Sigma, \mu=m_{f} V \Sigma}=p_{1}^{\text {latt. }}\left(\hat{\lambda}_{1} ; \hat{m}_{f}\right)
$$

The parameters $\hat{V}, \hat{\Sigma}$ and $\hat{m}_{f}$ are the dimensionless 4 -volume, the chiral condensate and the fermion mass of the SU(2) gauge theory in the lattice unit, respectively. Dimensionful quantities are $\lambda_{1}=\hat{\lambda}_{1} / a, V=a^{4} \hat{V}, \Sigma=\hat{\Sigma} / a^{3}$, and $m_{f}=\hat{m}_{f} / a$, where $a$ is the lattice spacing. The distribution of the smallest eigenvalue $p_{1}^{\text {latt. }}\left(\hat{\lambda}_{1} ; \hat{m}_{f}\right)$ is determined from lattice simulation and its normalization is fixed by

$$
\int_{0}^{\infty} d \hat{\lambda} p_{1}^{\text {latt. }}\left(\hat{\lambda} ; \hat{m}_{f}\right)=1
$$

\footnotetext{
${ }^{7}$ This equation is not valid if the lattice simulation is in the symmetric phase of the chiral symmetry, to which standard chiral RMT may not apply.
} 


\section{Nyström-type and $\mathrm{HMC}\left(\mathrm{N}_{\mathrm{f}}=8\right)$}

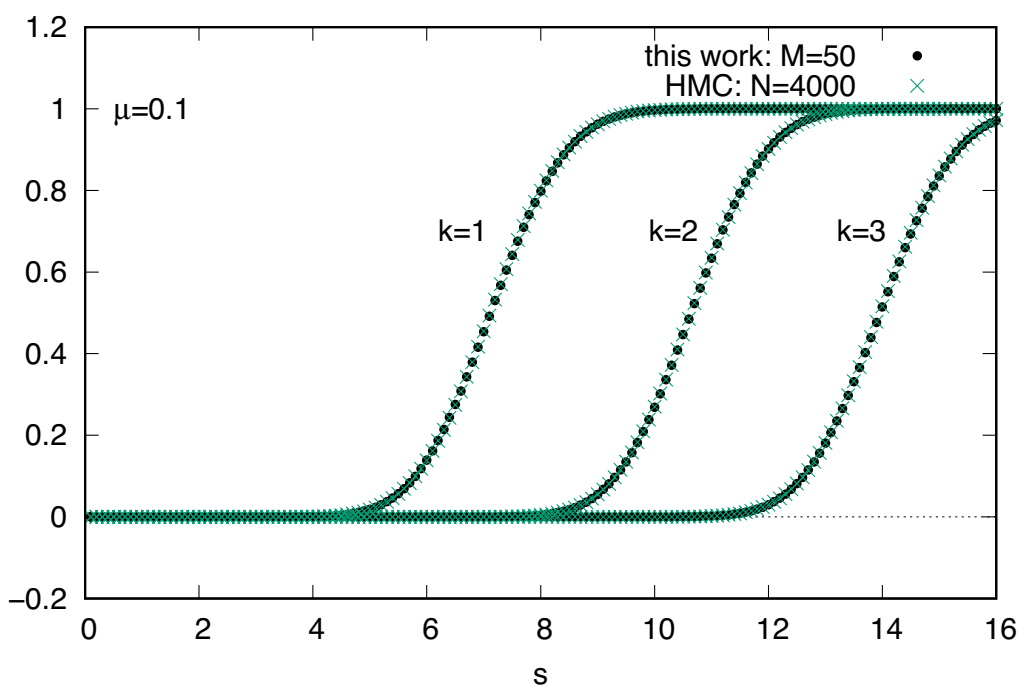

Figure 8. Plot of $F_{k}(s)$ in $0 \leq s \leq 16$ for $N_{F}=8$ in the complete confluent limit with $\mu_{1}=0.1$ and the topological charge $\nu=0$. Black dot: Nyström-type discretization of order $M=50$. Green cross: hybrid Monte Carlo simulation with the random matrix rank $N=4000$.

As the sole undetermined quantity in eq. (4.2) is the chiral condensate, we can use this relation to best-fit the value of $\hat{\Sigma}$. If the fit does not work, that is, if eq. (4.2) is not numerically satisfied by any choice of $\hat{\Sigma}$, it implies that the chiral symmetry is restored and the RM description is not applicable. Note that $\zeta_{1}$ and $\mu$ are dimensionless so they are directly related to quantities in the lattice unit: $\zeta_{1}=\lambda_{1} V \Sigma=\hat{\lambda}_{1} \hat{V} \hat{\Sigma}$ and $\mu=m_{f} V \Sigma=\hat{m}_{f} \hat{V} \hat{\Sigma}$. An integrated version of eq. (4.2) is

$$
F_{1}(s)=\left.\int_{0}^{s} d \zeta_{1} p_{1}^{\mathrm{RMT}}\left(\zeta_{1} ; \mu\right)\right|_{\zeta_{1}=\lambda_{1} V \Sigma, \mu=m_{f} V \Sigma}=\int_{0}^{\hat{s}} d \hat{\lambda}_{1} p_{1}^{\text {latt. }}\left(\hat{\lambda}_{1} ; \hat{m}_{f}\right) \quad(\equiv I(\hat{s})),
$$

where $s=\hat{s} \hat{V} \hat{\Sigma}$. We use $I(\hat{s})$ in the fitting process.

Our lattice setting is the following. We have three different lattice sizes, $(T / a) \times$ $(L / a)^{3}=8 \times 8^{3}, 12 \times 12^{3}$ and $16 \times 16^{3}$. In this paper, we use fermion mass $\hat{m}_{f}=a m_{f}=$ 0.010 . We use several values of the bare gauge coupling $\beta=4 / g^{2}$, for which we use $\beta=1.1-$ 1.475. These values are almost the same ones as used in [17]. See table 2 in appendix F for the details of the lattice data. The topological charge $\nu$ is calculated with the APE smeared [61] configuration with order- $a$ improved (i.e., "clover") field strength. Note that this gluonic definition does not give an integer value on a lattice. The obtained values, however, cluster around integer values so that we can identify configurations with $\nu=0$. Eigenvalues and topological charges are calculated for every 10 trajectories.

The details of our fitting procedure is as follows: We divide a given lattice eigenvalue distribution into $N_{\text {bin }}=25$ bins, whose support covers from 0 to 1.3 times the largest value in the distribution. In addition to the average value and the error in each bin, we estimate the correlation matrix $C$ between bins by using the jackknife method. Since a naive estimation of the correlation matrix causes unstable fitting, we use an improved 

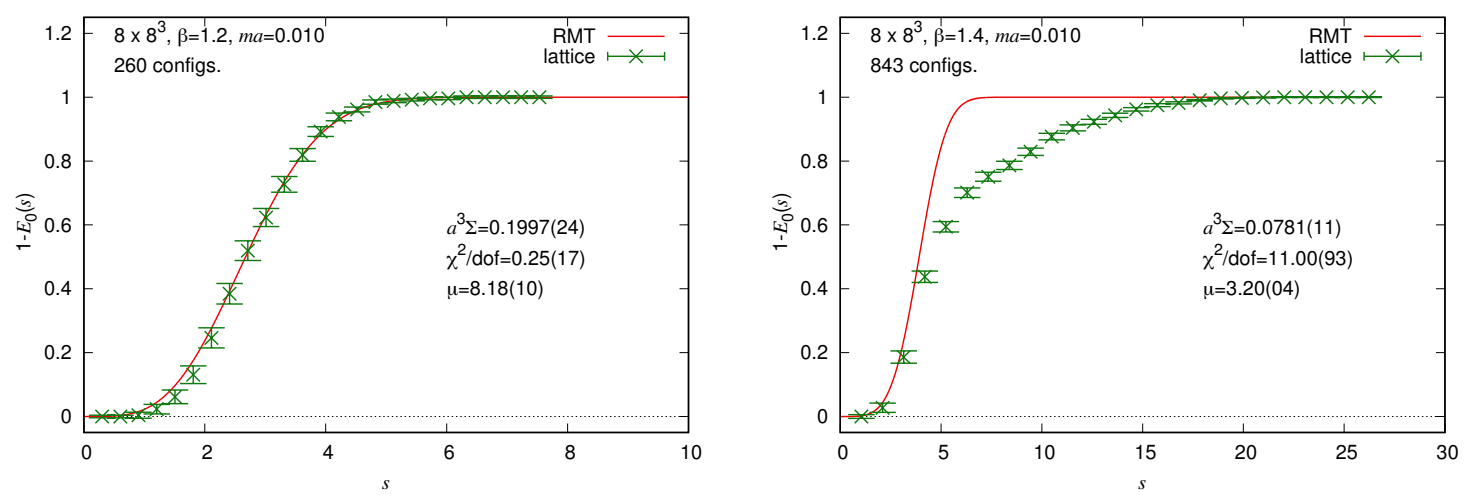

Figure 9. Typical example of a good fit (left) and a bad fit (right). The horizontal scale for the RMT curve is determined by the the best value of the chiral condensate, which is denoted in the plot.

estimation of the inverse, $C_{\mathrm{imp} .}^{-1}$. See appendix $\mathrm{G}$ for the details. The value of the chiral condensate $\hat{\Sigma}$ is determined by minimizing the correlated $\chi$ squared:

$$
\chi^{2}(\hat{\Sigma})=\sum_{i, j=1}^{N_{\text {bin }}}\left[I\left(\hat{s}_{i}\right)-I^{\mathrm{RMT}}\left(s_{i} ; \hat{\Sigma}\right)\right]\left(C_{\mathrm{imp}}^{-1}\right)_{i j}\left[I\left(\hat{s}_{j}\right)-I^{\mathrm{RMT}}\left(s_{j} ; \hat{\Sigma}\right)\right],
$$

where

$$
I^{\mathrm{RMT}}\left(s_{i} ; \hat{\Sigma}\right)=F_{1}\left(s_{i}\right), \quad \text { with } \quad s_{i}=\hat{s_{i}} \hat{V} \hat{\Sigma}, \quad \mu=\hat{V} \hat{\Sigma} \hat{m}_{f} .
$$

To estimate $p_{1}^{\mathrm{RMT}}\left(\zeta_{1}, \mu\right)$ with arbitrary $\zeta_{1}$ and $\mu$, which is needed to calculate $I^{\mathrm{RMT}}\left(s_{i} ; \hat{\Sigma}\right)$ for a given $\hat{\Sigma}$, we use interpolations in both $\zeta_{1}$ and $\mu$. We first interpolate in $\mu$ and then in $\zeta_{1}$, with the 4-point interpolation is used for both. Near the boundary of the available points where the 4-point interpolation is not possible, an interpolation with 3 points or an extrapolation with 2 points is used as well.

Figure 9 is a typical example of a good fit (indicating the chirally broken phase) and a bad fit (chirally symmetric phase). In the broken phase, the RMT well describes the smallest eigenvalue distribution from the lattice data, with a reasonably small value of $\chi$ squared. On the other hand, in the broken phase, the RMT curve can by no means describe the lattice data. In the figure, the plotted curve is the result with the best value of $\hat{\Sigma}=a^{3} \Sigma$. The value of $\chi$ squared, however, indicates that the quality of the fit is poor in the right panel and the RMT result is rejected as fitting ansatz.

It is interesting to note that even though the fit result is unreliable in the symmetric phase, the obtained value of the chiral condensate is small and consistent with zero, as should be in the symmetric phase. This is clearly seen in figure 10 . We observe that the larger the bare coupling $\beta=4 / g^{2}$ is, the smaller the obtained chiral condensate becomes and eventually the fit becomes unreliable near the vanishing of the chiral condensate at around $\beta=1.4-1.5$. In this figure, the unreliable data points, for which $\chi^{2}$ par degrees of freedom exceeds 1 , are plotted with pale colored symbols. Such behavior is also reported in [17], where the HMC with $N=400$ is used to obtain the RMT result. 


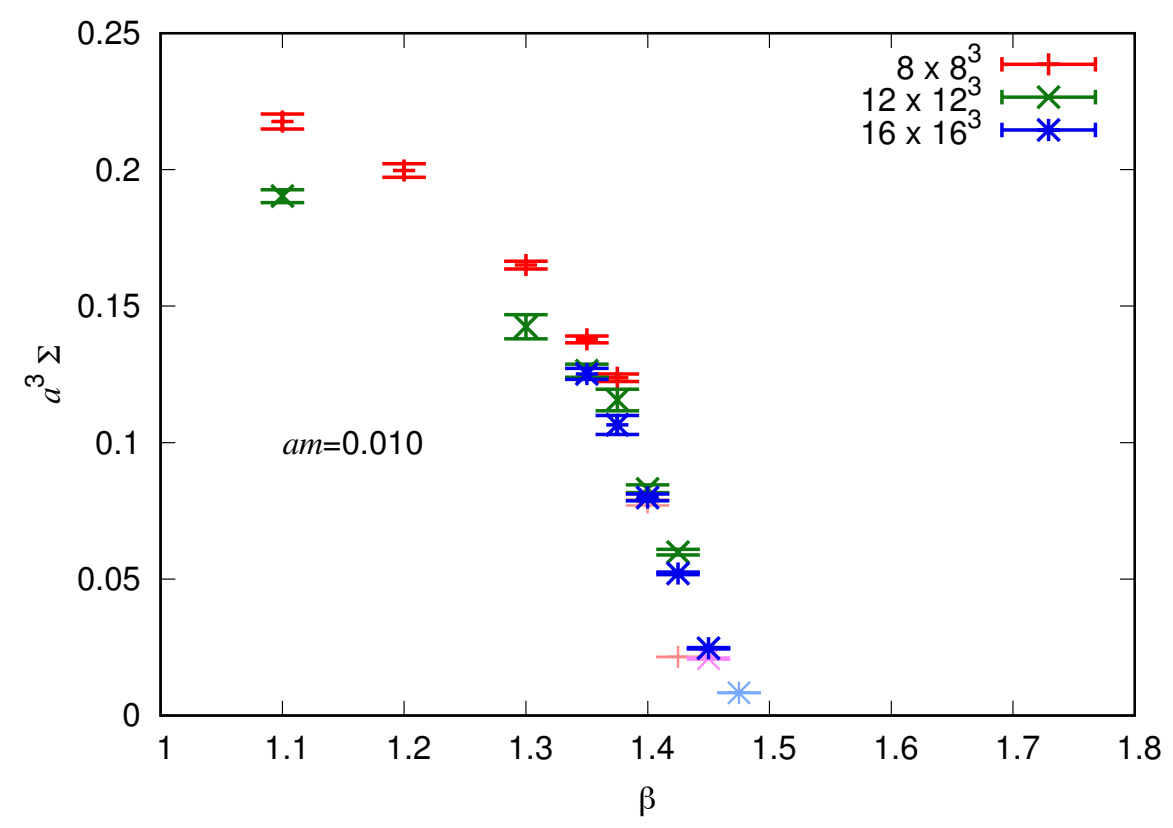

Figure 10. Chiral condensate versus bare coupling $\beta=4 / g_{0}^{2}$. The pale colored symbols have poor values of $\chi^{2} /$ d.o.f value $(>1)$.

\section{Conclusions and discussions}

We have numerically evaluated the $k^{\text {th }}$ smallest eigenvalue distributions of chiral random matrix ensembles with multiple flavors using the Nyström-type method applied to the Fredholm determinant and Pfaffian describing the Janossy densities. Adopting the compact determinant formulas (2.27) and (2.31) for the Fredholm determinant for the Janossy densities, we performed numerical computations for the chiral GUE and GSE in the asymptotic limit (2.9). One of our goals of these analyses is an application to the two-color QCD with $N_{F}$ fundamental staggered flavors. For the system of $N_{F}=8$ flavors in the fundamental representation of $\mathrm{SU}(2)$, the distribution of eigenvalues of the Dirac operators is being studied through the lattice simulation [17].

In the simulation we used, the taste symmetry of the staggered fermions is completely broken due to the finite lattice spacing, so that the remaining flavor symmetry is merely $N_{F}=2$. In addition to this flavor symmetry, due to the pseudo-reality of the fundamental representation of the $\mathrm{SU}(2)$ gauge group, all the eigenvalues of the Dirac operator are doubly degenerated. As a result, the distribution of the Dirac eigenvalues can fit with the chiral GSE with quadruply degenerated masses $N_{F}=4$ in the broken phase $\Sigma \neq 0$.

As shown in the left panel of figure 9 , we observed that the fitting with the chiral GSE works out very nicely in the broken phase. As the bare coupling $\beta=4 / g^{2}$ grows the chiral condensate becomes smaller and eventually the fitting becomes unreliable at around $\beta=1.4-1.5$ (figure 10). This implies that the chiral condensate vanishes and the symmetry is restored at $\beta \gtrsim 1.45$. A detailed analysis with more lattice data is currently ongoing [60]. We note that even with large values of the scaled quark masses $\mu_{f}$, fitting with 
the quenched chiral GSE is valid as long as the magnitude of the eigenvalue is much smaller than $F_{\pi}^{2} /(\Sigma \sqrt{V})$. Although the value of $F_{\pi}$, the pion decay constant, is not available from the current lattice data, it is natural to assume that the smallest of the Dirac eigenvalues satisfies this condition in the broken phase. The Banks-Casher relation tells us that the smallest eigenvalue is small enough to give a non-zero eigenvalue density around the origin.

Finally we will list some directions for the future research. Firstly, the numerical computations developed in this article could also be applied to the two-color QCD with $N_{F}=8-12$ fundamental flavors. Among such systems, the existence of the conformal window is strongly expected, and the technology of their lattice simulations is developping remarkably in recent years. We anticipate that the RMT analysis of the spectral statistics of the Dirac operators would discriminate the (near-)conformality of the QCD-like systems and unveil some novel aspects of the conformal window.

The Fredholm Pfaffian for the Janossy density of the chiral Gaussian orthogonal ensemble will deserve a future study direction; the chiral GOE describes the distributions of the Dirac eigenvalues for QCD-like systems with staggered fermions in adjoint representation of $\mathrm{SU}\left(N_{c}\right)$ [21]. It is known that the exponential convergence of the Nyström-type discretization of the Fredholm Pfaffian for the orthogonal ensemble is not guaranteed due to the infinite oscillations originating from the discontinuity of the quaternion kernel elements. Even though such hard problem resides, we may still be able to apply the Nyström-type discretization for the practical purpose if the error can be estimated appropriately, and use it to estimate the value of the chiral condensate $\Sigma$ for the adjoint QCD-like system. ${ }^{8}$

In $[54,55]$, an exact analysis of the Janossy density for the unitary ensemble is done on a basis of the Painlevé II transcendent and its associated isomonodromic system. Generalization of such an exact analysis to the symplectic and orthogonal ensembles could be an interesting direction yet to be studied, and it can be compared with our numerical results.

Recent years, the $(0+1)$-dimensional fermionic model with all-to-all random interactions referred to as Sachdev-Ye-Kitaev (SYK) model [64, 65] has been studied very actively in the context of the nonequilibrium quantum many-body systems and its application to the AdS/CFT correspondence (see references in a recent review article [66]). The level statistics of the SYK model was numerically examined, and good agreements with the RMT have been observed. It would be interesting to explore how the Fredholm determinant or Pfaffian expression for the Janossy density of the chiral random matrices appears in the level statistics of the supersymmetric SYK Hamiltonian [67, 68].

\section{Acknowledgments}

The authors would like to thank Peter J. Forrester, C. -J. David Lin, and Taro Nagao for fruitful discussions and valuable comments.

The authors thank Asia Pacific Center for Theoretical Physics (APCTP) where this work was initiated during the Workshop 2017 on "Discrete Approaches to the Dynamics of Fields and Space-Time". The authors also thank the Yukawa Institute for Theoretical

\footnotetext{
${ }^{8}$ The analytical computations of the smallest eigenvalue distribution $(k=1)$ for the chiral GOE are found in $[62,63]$.
} 
Physics (YITP) at Kyoto University, for the discussions during the Workshop YITP-S-1704 on "The 40th Shikoku Seminar on Particle and Nuclear Physics" which was useful to complete this work.

The research of H.F. is supported by the Grant-in-Aid for Challenging Research (Exploratory) [\# 17K18781], Grant-in-Aid for Scientific Research(C) [\# 17K05239], [\# 18K03281], and Grant-in-Aid for Scientific Research(B) [\# 16H03927] from the Japan Ministry of Education, Culture, Sports, Science and Technology. The research of I.K. is supported by MEXT as "Priority Issue 9 to be Tackled by Using Post-K Computer" (Elucidation of the Fundamental Laws and Evolution of the Universe) and JICFuS. The research of S.M.N. is supported by Grants-in-Aid for Scientific Research(C) [\# 25400259] and [\# 17K05416]. National Centre for High-performance Computing and National Chiao-Tung University (NCTU) HPC facility in Taiwan were used for lattice simulation.

\section{A Quaternionic kernels for the chiral Gaussian symplectic ensemble}

In this appendix, the explicit forms of the spectral kernel for the chiral GSE are summarized for quadruply degenerated masses $N_{F}=4 \alpha$ and doubly degenerated masses $N_{F}=2 \alpha$.

\section{A.1 Spectral kernel for quadruply degenerated masses}

The scaled correlation function of the $\beta=4$ chiral RMT with quadruply degenerated masses $N_{F}=4 \alpha$ in the scaling limit (2.9) is given in [30].

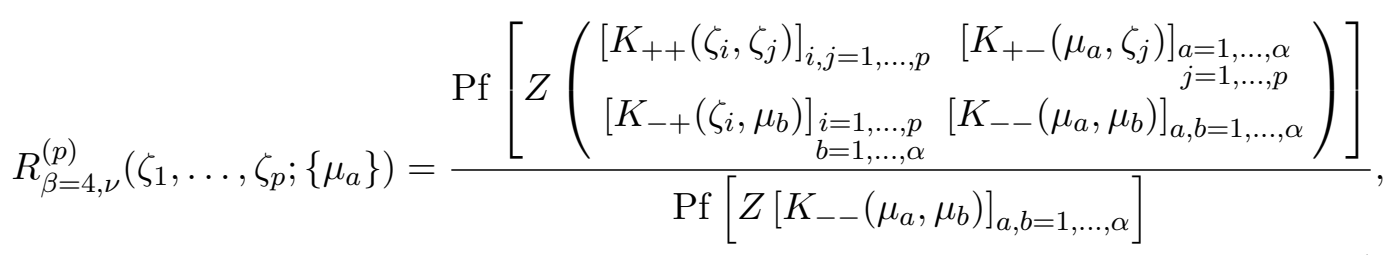

$$
\begin{aligned}
& K_{A B}\left(\zeta, \zeta^{\prime}\right)=\left[\begin{array}{cc}
-S_{A B}\left(\zeta, \zeta^{\prime}\right) & -I_{A B}\left(\zeta, \zeta^{\prime}\right) \\
D_{A B}\left(\zeta, \zeta^{\prime}\right) & -S_{B A}\left(\zeta^{\prime}, \zeta\right)
\end{array}\right], \quad Z=\mathrm{i} \sigma_{2} \otimes \mathbb{I},
\end{aligned}
$$

where elements of block matrices are

$$
\begin{aligned}
& S_{++}\left(\zeta, \zeta^{\prime}\right)=2 \int_{0}^{1} d u \int_{0}^{1} d \sqrt{\zeta \zeta^{\prime} \zeta v^{2}} \\
& \times\left(J_{2 \nu}(2 v \zeta) u J_{2 \nu+1}\left(2 u v \zeta^{\prime}\right)-J_{2 \nu}(2 u v \zeta) J_{2 \nu+1}\left(2 v \zeta^{\prime}\right)\right), \\
& S_{+-}\left(\zeta, \eta^{\prime}\right)=(-1)^{\nu+1} 2 \int_{0}^{1} d u \int_{0}^{1} d v \sqrt{\zeta \eta^{\prime}} \zeta v^{2} \\
& \times\left(J_{2 \nu}(2 v \zeta) u I_{2 \nu+1}\left(2 u v \eta^{\prime}\right)-J_{2 \nu}(2 u v \zeta) I_{2 \nu+1}\left(2 v \eta^{\prime}\right)\right), \\
& S_{-+}\left(\eta, \zeta^{\prime}\right)=(-1)^{\nu+1} 2 \int_{0}^{1} d u \int_{0}^{1} d v \sqrt{\eta \zeta^{\prime}} \eta v^{2} \\
& \times\left(I_{2 \nu}(2 v \eta) u J_{2 \nu+1}\left(2 u v \zeta^{\prime}\right)-I_{2 \nu}(2 u v \eta) J_{2 \nu+1}\left(2 v \zeta^{\prime}\right)\right),
\end{aligned}
$$




$$
\begin{aligned}
& S_{--}\left(\eta, \eta^{\prime}\right)=(-1)^{2 \nu} 2 \int_{0}^{1} d u \int_{0}^{1} d v \sqrt{\eta \eta^{\prime}} \eta v^{2} \\
& \times\left(I_{2 \nu}(2 v \eta) u I_{2 \nu+1}\left(2 u v \eta^{\prime}\right)-I_{2 \nu}(2 u v \eta) I_{2 \nu+1}\left(2 v \eta^{\prime}\right)\right), \\
& D_{++}\left(\zeta, \zeta^{\prime}\right)=2 \int_{0}^{1} d u \int_{0}^{1} d v \sqrt{\zeta \zeta^{\prime}} v^{3} u \\
& \times\left(J_{2 \nu+1}(2 v \zeta) J_{2 \nu+1}\left(2 u v \zeta^{\prime}\right)-J_{2 \nu+1}(2 u v \zeta) J_{2 \nu+1}\left(2 v \zeta^{\prime}\right)\right), \\
& D_{+-}\left(\zeta, \eta^{\prime}\right)=(-1)^{\nu+1} 2 \int_{0}^{1} d u \int_{0}^{1} d v \sqrt{\zeta \eta^{\prime}} v^{3} u \\
& \times\left(J_{2 \nu+1}(2 v \zeta) I_{2 \nu+1}\left(2 u v \eta^{\prime}\right)-J_{2 \nu+1}(2 u v \zeta) I_{2 \nu+1}\left(2 v \eta^{\prime}\right)\right), \\
& D_{-+}\left(\eta, \zeta^{\prime}\right)=-D_{+-}\left(\zeta^{\prime}, \eta\right), \\
& D_{--}\left(\eta, \eta^{\prime}\right)=(-1)^{2 \nu} 2 \int_{0}^{1} d u \int_{0}^{1} d v \sqrt{\eta \eta^{\prime}} v^{3} u \\
& \times\left(I_{2 \nu+1}(2 v \eta) I_{2 \nu+1}\left(2 u v \eta^{\prime}\right)-I_{2 \nu+1}(2 u v \eta) I_{2 \nu+1}\left(2 v \eta^{\prime}\right)\right), \\
& I_{++}\left(\zeta, \zeta^{\prime}\right)=2 \int_{0}^{1} d u \int_{0}^{1} d v \sqrt{\zeta \zeta^{\prime}} \zeta \zeta^{\prime} v\left(J_{2 \nu}(2 v \zeta) J_{2 \nu}\left(2 u v \zeta^{\prime}\right)-J_{2 \nu}(2 u v \zeta) J_{2 \nu}\left(2 v \zeta^{\prime}\right)\right) \text {, } \\
& I_{+-}\left(\zeta, \eta^{\prime}\right)=(-1)^{\nu+1} 2 \int_{0}^{1} d u \int_{0}^{1} d v \sqrt{\zeta \eta^{\prime}} \zeta \eta^{\prime} v\left(J_{2 \nu}(2 v \zeta) I_{2 \nu}\left(2 u v \eta^{\prime}\right)-J_{2 \nu}(2 u v \zeta) I_{2 \nu}\left(2 v \eta^{\prime}\right)\right) \\
& I_{-+}\left(\zeta, \eta^{\prime}\right)=-I_{+-}\left(\eta^{\prime}, \zeta\right) \\
& I_{--}\left(\eta, \eta^{\prime}\right)=(-1)^{2 \nu} 2 \int_{0}^{1} d u \int_{0}^{1} d v \sqrt{\eta \eta^{\prime}} \eta \eta^{\prime} v\left(I_{2 \nu}(2 v \eta) I_{2 \nu}\left(2 u v \zeta^{\prime}\right)-I_{2 \nu}(2 u v \eta) I_{2 \nu}\left(2 v \eta^{\prime}\right)\right) .
\end{aligned}
$$

\section{A.2 Spectral kernel for doubly degenerated masses}

The $p$-level correlation function for $\beta=4$ chiral RMT with $N_{F}=2 \alpha$ doubly degenerated masses in the scaling limit (2.9) is given in [30].

$$
R_{\beta=4, \nu}^{(p)}\left(\zeta_{1}, \ldots, \zeta_{p} ;\left\{\mu_{a}\right\}\right)=\frac{\operatorname{Pf}\left[Z K^{(p)}\right]}{\operatorname{Pf}\left[Z K^{(0)}\right]} .
$$

For even $\alpha$, the kernel $Z K^{(p)}$ is given as follows:

$$
Z K^{(p)}=\left(\begin{array}{ccc}
{\left[I_{--}\left(\mu_{a}, \mu_{b}\right)\right]_{a, b=1, \ldots, \alpha}} & {\left[I_{-+}\left(\mu_{a}, \zeta_{i}\right)\right]_{\substack{a=1, \ldots, \alpha \\
j=1, \ldots, p}}} & {\left[S_{-+}\left(\mu_{a}, \zeta_{j}\right)\right]_{\substack{a=1, \ldots, \alpha \\
j=1, \ldots, p}}} \\
-\left[I_{-+}^{\mathrm{T}}\left(\mu_{b}, \zeta_{i}\right)\right]_{\substack{b=1, \ldots, \alpha \\
i=1, \ldots, p}} & {\left[I_{++}\left(\zeta_{i}, \zeta_{j}\right)\right]_{i, j=1, \ldots, p}} & {\left[S_{++}\left(\zeta_{i}, \zeta_{j}\right)\right]_{i, j=1, \ldots, p}} \\
-\left[S_{-+}^{\mathrm{T}}\left(\mu_{b}, \zeta_{i}\right)\right]_{\substack{b=1, \ldots, \alpha \\
i=1, \ldots, p}} & -\left[S_{++}^{\mathrm{T}}\left(\zeta_{j}, \zeta_{i}\right)\right]_{i, j=1, \ldots, p} & {\left[D_{++}\left(\zeta_{i}, \zeta_{j}\right)\right]_{i, j=1, \ldots, p}}
\end{array}\right)
$$

where $S_{A B}$ 's, $D_{A B}$ 's, and $I_{A B}$ 's are the same as $N_{F}=4 \alpha$ in appendix A.1, and T stands for the transposition of the block matrix. 
For odd $\alpha$, the kernel $Z K^{(p)}$ is given as follows:

$$
Z K^{(p)}=\left(\begin{array}{cccc}
{\left[I_{--}\left(\mu_{a}, \mu_{b}\right)\right]_{a, b=1, \ldots, \alpha}} & {\left[Q_{-}\left(\mu_{a}\right)\right]_{a=1, \ldots, \alpha}} & {\left[I_{-+}\left(\mu_{a}, \zeta_{j}\right)\right]_{a=1, \ldots, \alpha}} & {\left[S_{-+}\left(\mu_{a}, \zeta_{j}\right)\right]_{a=1, \ldots, \alpha}} \\
-\left[Q_{-}^{\mathrm{T}}\left(\mu_{b}\right)\right]_{b=1, \ldots, \alpha} & 0 & -\left[Q_{+}^{\mathrm{T}}\left(\zeta_{j}\right)\right]_{j=1, \ldots, p} & -\left[P_{+}^{\mathrm{T}}\left(\zeta_{j}\right)\right]_{j=1, \ldots, p} \\
-\left[I_{-+}^{\mathrm{T}}\left(\mu_{b}, \zeta_{i}\right)\right]_{i=1, \ldots, p} & {\left[Q_{+}\left(\zeta_{i}\right)\right]_{i=1, \ldots, p}} & {\left[I_{++}\left(\zeta_{i}, \zeta_{j}\right)\right]_{i, j=1, \ldots, p}} & {\left[S_{++}\left(\zeta_{i}, \zeta_{j}\right)\right]_{i, j=1, \ldots, p}} \\
-\left[S_{-+}^{\mathrm{T}}\left(\mu_{b}, \zeta_{i}\right)\right]_{i=1, \ldots, p} & {\left[P_{+}\left(\zeta_{i}\right)\right]_{i=1, \ldots, p}} & -\left[S_{++}^{\mathrm{T}}\left(\zeta_{j}, \zeta_{i}\right)\right]_{i, j=1, \ldots, p} & {\left[D_{++}\left(\zeta_{i}, \zeta_{j}\right)\right]_{i, j=1, \ldots, p}}
\end{array}\right) .
$$

where $S_{A B}$ 's, $D_{A B}$ 's, and $I_{A B}$ 's are the same as $N_{F}=4 \alpha$ in appendix A.1, and

$$
\begin{aligned}
& Q_{+}(\zeta)=2 \sqrt{\zeta} \zeta \int_{0}^{1} d v J_{2 \nu}(2 v \zeta), \quad Q_{-}(\eta)=(-1)^{\nu+1} 2 \sqrt{\eta} \eta \int_{0}^{1} d v I_{2 \nu}(2 v \eta), \\
& P_{+}(\zeta)=2 \sqrt{\zeta} \int_{0}^{1} d v v J_{2 \nu+1}(2 v \zeta)
\end{aligned}
$$

\section{B Confluent limits of the correlation function}

\section{B.1 Chiral Gaussian unitary ensemble}

Let $Z_{\beta=2, \nu}\left(x_{1}, \ldots, x_{n}\right)$ be the partition function which is obtained as the scaling limit (2.9) of the chiral Gaussian unitary ensemble with $N_{f}=2 n$ mass parameters $x_{a}=m_{a} / \Delta[18$, $26,27]$.

$$
Z_{\beta=2, \nu}\left(x_{1}, \ldots, x_{n}\right)=\frac{\operatorname{det}\left[x_{a}^{b-1} I_{\nu+b-1}\left(x_{a}\right)\right]_{a, b=1}^{n}}{\prod_{a>b}\left(x_{a}^{2}-x_{b}^{2}\right)} .
$$

To consider the confluent limit $x_{i} \rightarrow x_{j}$ of this partition function [69], we will use the l' Hôpital's rule given as follows.

Let $f, g$ be differentiable functions on an interval $I \in \mathbb{R}$. Assume that for $c \in I$, (1) $\lim _{x \rightarrow c} f(x)=\lim _{x \rightarrow c} g(x)=0$ or $\infty,(2) \lim _{x \rightarrow c} f^{\prime}(x) / g^{\prime}(x)$ exists, (3) $g^{\prime}(x) \neq 0$ for $x \in I \backslash\{c\}$, then

$$
\lim _{x \rightarrow c} \frac{f(x)}{g(x)}=\lim _{x \rightarrow c} \frac{f^{\prime}(x)}{g^{\prime}(x)} .
$$

One finds that the confluent limit $x_{i} \rightarrow x_{1}=x(i=1, \ldots, n)$ of the partition function $Z_{\beta=2, \nu}\left(x_{1}, \ldots, x_{n}\right)$ by adopting the l' Hôpital's rule (B.2) repeatedly.

$$
\begin{aligned}
& \lim _{x_{n} \rightarrow x_{1}} \lim _{x_{n-1} \rightarrow x_{1}} \ldots \lim _{x_{2} \rightarrow x_{1}} Z_{\beta=2, \nu}\left(x_{1}, \ldots, x_{n}\right) \\
& =\lim _{x_{n} \rightarrow x_{1}} \lim _{x_{n-1} \rightarrow x_{1}} \cdots \lim _{x_{2} \rightarrow x_{1}} \frac{\left|\begin{array}{cccc}
I_{0}\left(x_{1}\right) & x_{1} I_{1}\left(x_{1}\right) & \cdots & x_{1}^{n-1} I_{n-1}\left(x_{1}\right) \\
I_{0}\left(x_{2}\right) & x_{2} I_{1}\left(x_{2}\right) & \cdots & x_{2}^{n-1} I_{n-1}\left(x_{2}\right) \\
\vdots & \vdots & \vdots & \vdots \\
I_{0}\left(x_{n}\right) & x_{n} I_{1}\left(x_{n}\right) & \cdots & x_{n}^{n-1} I_{n-1}\left(x_{n}\right)
\end{array}\right|}{\left(x_{1}^{2}-x_{2}^{2}\right)\left(x_{1}^{2}-x_{2}^{2}\right) \cdots\left(x_{n-1}^{2}-x_{n}^{2}\right)}
\end{aligned}
$$




$$
\begin{aligned}
& =\left(\frac{\frac{1}{2} \frac{1}{2^{2}} \frac{1}{2^{3}} \cdots \frac{1}{2^{n-1}}}{1 ! 2 ! 3 ! \cdots(n-1) !}\right)\left|\begin{array}{cccc}
I_{0}\left(x_{1}\right) & x_{1} I_{1}\left(x_{1}\right) & \cdots & x_{1}^{n-1} I_{n-1}\left(x_{1}\right) \\
x_{1}^{-1} I_{-1}\left(x_{1}\right) & I_{0}\left(x_{1}\right) & \cdots & x_{1}^{n-2} I_{n-2}\left(x_{1}\right) \\
x_{1}^{-2} I_{-2}\left(x_{1}\right) & x_{1}^{-1} I_{-1}\left(x_{1}\right) & \cdots & x_{1}^{n-3} I_{n-3}\left(x_{1}\right) \\
\vdots & \vdots & \vdots & \vdots \\
x_{1}^{-(n-1)} I_{-(n-1)}\left(x_{1}\right) & x_{1}^{-(n-2)} I_{-(n-2)}\left(x_{1}\right) & \cdots & x_{1}^{0} I_{0}\left(x_{1}\right)
\end{array}\right| \\
& =\frac{\frac{1}{2} \frac{1}{2^{2}} \frac{1}{2^{3}} \cdots \frac{1}{2^{n-1}}}{1 ! 2 ! 3 ! \cdots(n-1) !} \operatorname{det}\left[x_{1}^{a-b} I_{a-b}(x)\right]_{a, b=1}^{n},
\end{aligned}
$$

where the following formula of the Bessel function is adopted

$$
2 \frac{\partial}{\partial x^{2}}\left(x^{k} I_{k}(x)\right)=x^{k-1} I_{k-1}(x) .
$$

Next we will consider the scalar kernel $K_{s}\left(\zeta, \zeta^{\prime}, \mu_{1}, \mu_{2}, \ldots, \mu_{\alpha}\right)$ for the chiral GUE with $\nu=0[18,26,27]$.

$$
\begin{aligned}
K_{s}\left(\zeta, \zeta^{\prime}, \mu_{1}, \mu_{2}, \ldots, \mu_{\alpha}\right)= & \frac{\sqrt{\zeta \zeta^{\prime}}}{\left(\zeta^{2}-\zeta^{\prime 2}\right) \prod_{k=1}^{\alpha} \sqrt{\left(\zeta^{2}+\mu_{k}^{2}\right)\left(\zeta^{\prime 2}+\mu_{k}^{2}\right)}} \\
& \times \frac{\left|\begin{array}{cccc}
J_{0}(\zeta) & \zeta J_{1}(\zeta) & \cdots & \zeta^{\alpha+1} J_{\alpha+1}(\zeta) \\
J_{0}\left(\zeta^{\prime}\right) & \zeta J_{1}\left(\zeta^{\prime}\right) & \cdots & \zeta^{\prime \alpha+1} J_{\alpha+1}\left(\zeta^{\prime}\right) \\
I_{0}\left(\mu_{1}\right) & \zeta\left(-\mu_{1}\right) I_{1}\left(\mu_{1}\right) & \cdots & \mu_{1}^{\alpha+1} J_{\alpha+1}\left(\mu_{1}\right) \\
\vdots & \vdots & \cdots & \vdots \\
I_{0}\left(\mu_{\alpha}\right) & \zeta\left(-\mu_{\alpha}\right) I_{1}\left(\mu_{\alpha}\right) & \cdots & \mu_{\alpha}^{\alpha+1} J_{\alpha+1}\left(\mu_{\alpha}\right)
\end{array}\right|}{\operatorname{det}\left[\left(-\mu_{k}\right)^{\ell} I_{\ell}\left(\mu_{k}\right)\right]_{k, \ell=1}^{\alpha}}
\end{aligned}
$$

The confluent limit of $K_{s}\left(\zeta, \zeta^{\prime}, \mu_{1}, \mu_{2}, \ldots, \mu_{\alpha}\right)$ is also obtained in the same way as the partition function $Z_{\beta=2, \nu}\left(x_{1}, \ldots, x_{n}\right)$ considered above. For our notational convenience, we introduce

$$
A_{k}(x)=x^{k} I_{k}(x), \quad B_{k}(x)=(-x)^{k} J_{k}(x)
$$

and $A_{k}$ 's obey

$$
\frac{d}{d x^{2}} A_{k}(x)=\frac{1}{2} A_{k-1} .
$$

Adopting such notation, one can express the complete confluent limit $\left(\mu_{i} \rightarrow \mu_{1}=\mu\right.$ for $i=2, \ldots, \alpha)$ of the determinant factor in the scalar kernel $K_{s}\left(\zeta, \zeta^{\prime}, \mu_{1}, \mu_{2}, \ldots, \mu_{\alpha}\right)$.

$\lim _{\mu_{2} \rightarrow \mu_{1}} \cdots \lim _{\mu_{\alpha} \rightarrow \mu_{1}} \frac{\left|\begin{array}{cccc}B_{0}(\zeta) & B_{1}(\zeta) & \cdots & B_{\alpha+1}(\zeta) \\ B_{0}\left(\zeta^{\prime}\right) & B_{1}\left(\zeta^{\prime}\right) & \cdots & B_{\alpha+1}\left(\zeta^{\prime}\right) \\ A_{0}\left(\mu_{1}\right) & A_{1}\left(\mu_{1}\right) & \cdots & A_{\alpha+1}\left(\mu_{1}\right) \\ A_{0}\left(\mu_{2}\right) & A_{1}\left(\mu_{2}\right) & \cdots & A_{\alpha+1}\left(\mu_{2}\right) \\ \vdots & \vdots & \cdots & \vdots \\ A_{0}\left(\mu_{\alpha}\right) & A_{1}\left(\mu_{\alpha}\right) & \cdots & A_{\alpha+1}\left(\mu_{\alpha}\right)\end{array}\right|}{\operatorname{det}\left[A_{\ell}\left(\mu_{k}\right)\right]_{k, \ell=1}^{\alpha}}=\frac{\left|\begin{array}{cccc}B_{0}(\zeta) & B_{1}(\zeta) & \cdots & B_{\alpha+1}(\zeta) \\ B_{0}\left(\zeta^{\prime}\right) & B_{1}\left(\zeta^{\prime}\right) & \cdots & B_{\alpha+1}\left(\zeta^{\prime}\right) \\ A_{0}(\mu) & A_{1}(\mu) & \cdots & A_{\alpha+1}(\mu) \\ A_{-1}(\mu) & A_{0}(\mu) & \cdots & A_{\alpha}(\mu) \\ \vdots & \vdots & \cdots & \vdots \\ A_{-\alpha+1}(\mu) & A_{-\alpha+2}(\mu) & \cdots & A_{0}(\mu)\end{array}\right|}{\operatorname{det}\left[A_{k-\ell}(\mu)\right]_{k, \ell=1}^{\alpha}}$ 
Completed by the confluent limit of remaining factors in $K_{s}\left(\zeta, \zeta^{\prime}, \mu_{1}, \mu_{2}, \ldots, \mu_{\alpha}\right)$, one obtains the confluent limit of the spectral kernel for the chiral GUE.

\section{B.2 Chiral Gaussian symplectic ensemble}

The partition function $Z_{\beta=4, \nu}\left(\left\{\mu_{a}\right\}\right)$ for the scaling limit (2.9) of the chiral GSE $(\beta=4)$ with $2 \alpha$ ( $\alpha$ : even) flavors of the doubly degenerated masses is given as follows [30].

$$
Z_{\beta=4, \nu}\left(\left\{\mu_{a}\right\}\right)=c_{\alpha}\left(\prod_{i=1}^{\alpha} \mu_{i}^{2 \nu}\right) \frac{\operatorname{Pf}(Z f)}{\Delta\left(\mu_{1}^{2}, \ldots, \mu_{\alpha}^{2}\right)},
$$

where

$$
\begin{aligned}
& c_{\alpha}=(-1)^{\frac{\alpha(\alpha+1)}{2}} \prod_{k=0}^{\alpha-1}(2 k+1) !, \quad \Delta\left(\mu_{1}^{2}, \ldots, \mu_{\alpha}^{2}\right)=\prod_{i>j}\left(\mu_{i}^{2}-\mu_{j}^{2}\right) . \\
& f_{i j}=f\left(\mu_{i}, \mu_{j}\right)=\int_{0}^{1} d t t \frac{I_{2 \nu}\left(2 t \mu_{i}\right)}{\mu_{i}^{\nu}} \int_{0}^{1} d u \frac{I_{2 \nu}\left(2 t u \mu_{j}\right)}{\mu_{j}^{2 \nu}}-(i \leftrightarrow j) .
\end{aligned}
$$

The complete confluent limit $\mu_{i} \rightarrow \mu_{1}=\mu$ of the partition function $Z_{\beta=4, \nu}\left(\mu_{1}^{\otimes 2}, \ldots, \mu_{n}^{\otimes 2}\right)$ yields

$$
\lim _{\mu_{2, \ldots, n} \rightarrow \mu_{1}=\mu} Z_{\beta=4, \nu}\left(\mu_{1}^{\otimes 2}, \ldots, \mu_{n}^{\otimes 2}\right)=\frac{\frac{1}{2} \frac{1}{2^{2}} \cdots \frac{1}{2^{n-1}}}{1 ! 2 ! \cdots(n-1) !} c_{4} \cdot \operatorname{Pf}\left(Z\left[f^{(i, j)}(\mu, \mu)\right]_{i, j=0}^{n-1}\right),
$$

where

$$
f^{(k, \ell)}\left(\mu_{i}, \mu_{j}\right)=\left(\frac{\partial}{\partial \mu_{i}^{2}}\right)^{k}\left(\frac{\partial}{\partial \mu_{j}^{2}}\right)^{\ell} f\left(\mu_{i}, \mu_{j}\right) .
$$

The complete confluent limit $\left(\mu_{i} \rightarrow \mu_{1}=\mu\right.$ for $\left.i=2, \ldots, \alpha\right)$ of the correlation function (A.6) in [30] is also obtained in the same way. In the limit $\mu_{2, \ldots, n} \rightarrow \mu_{1}=\mu$, matrix elements in eq. (A.6) are replaced in the following way. (For simplicity, we consider the case of $\nu=0$.)

$$
\begin{gathered}
I_{--}\left(\mu_{i}, \mu_{j}\right) \rightarrow I_{--}^{(i-1, j-1)}(\mu, \mu) \\
=\mu^{2} \int_{0}^{1} d t \int_{0}^{1} d u t\left[(2 t)^{2(i-1)}(2 t u)^{2(j-1)} A_{-i+1}(2 t \mu) A_{-j+1}(2 t u \mu)\right. \\
\left.-(2 t)^{2(j-1)}(2 t u)^{2(i-1)} A_{-j+1}(2 t \mu) A_{-i+1}(2 t u \mu)\right], \\
I_{-+}\left(\mu_{i}, \zeta_{\ell}\right) \rightarrow I_{-+}^{(i-1,0)}\left(\mu, \zeta_{\ell}\right) \\
=\mu \int_{0}^{1} d t \int_{0}^{1} d u t\left[(2 t)^{2(i-1)} A_{-i+1}(2 t \mu) J_{0}\left(2 t u \zeta_{\ell}\right)\right. \\
S_{-+}\left(\mu_{i}, \zeta_{\ell}\right) \rightarrow S_{-+}^{(i-1,0)}\left(\mu, \zeta_{\ell}\right) \\
=\mu \int_{0}^{1} d t \int_{0}^{1} d u t^{2}\left[(2 t)^{2(i-1)} u A_{-i+1}(2 t \mu) J_{1}\left(2 t u \zeta_{\ell}\right)\right. \\
\left.-(2 t u)^{2(i-1)} A_{-i+1}(2 t u \mu) J_{1}\left(2 t \zeta_{\ell}\right)\right] .
\end{gathered}
$$


The other matrix elements are kept untouched, because they are not dependent on mass parameters $\mu_{i}$ 's.

Lastly, for the odd $\alpha$ case in addition to the above replacements, the matrix elements $Q_{-}$'s in eq. (A.7) are also replaced by

$$
Q_{-}\left(\mu_{j}\right) \rightarrow Q_{-}^{(j-1)}(\mu)=\frac{\partial^{i-1}}{\partial\left(\mu^{2}\right)^{j-1}} Q_{-}(\mu)=\left.\int_{0}^{1} d v(2 v)^{2(j-1)-1} \frac{d^{j-1}}{d\left(x^{2}\right)^{j-1}}\left(x A_{0}(x)\right)\right|_{x=2 v \mu} .
$$

\section{B.2.1 Quadruply degenerated kernels in the confluent limit}

For the chiral GSE with $N_{F}=4 \alpha$ quadruply degenerated masses, we can use the spectral kernel given in appendix A.1. In the complete confluent limit, some matrix elements in eqs. (A.2)-(A.4) are replaced as follows. (We also choose $\nu=0$ for simplicity.)

$$
\begin{aligned}
& S_{+-}\left(\zeta, \mu_{a}\right) \rightarrow S_{+-}^{(0, a-1)}(\zeta, \mu) \\
& =2 \int_{0}^{1} d u \int_{0}^{1} d v \sqrt{\zeta \mu} \zeta v^{2} \\
& \times\left[\left.(2 u v)^{2(a-1)} u J_{0}(2 v \zeta) \frac{d^{a-1}}{d\left(x^{2}\right)^{a-1}}\left(x^{-1} A_{1}(x)\right)\right|_{x=2 u v \mu}\right. \\
& \left.-\left.(2 v)^{2(a-1)} J_{0}(2 u v \zeta) \frac{d^{a-1}}{d\left(x^{2}\right)^{a-1}}\left(x^{-1} A_{1}(x)\right)\right|_{x=2 v \mu}\right], \\
& S_{-+}\left(\mu_{a}, \zeta\right) \rightarrow S_{-+}^{(0, a-1)}(\zeta, \mu) \\
& =2 \int_{0}^{1} d u \int_{0}^{1} d v \sqrt{\zeta \mu} \mu v^{2}\left[(2 v)^{2(a-1)} u A_{-a+1}(2 v \mu) J_{1}(2 u v \mu)\right. \\
& \left.-(2 u v)^{2(a-1)} A_{-a+1}(2 u v \mu) J_{1}(2 v \mu)\right], \\
& S_{--}\left(\mu_{a}, \mu_{b}\right) \rightarrow S_{--}^{(a-1, b-1)}(\mu, \mu) \\
& =-2 \int_{0}^{1} d u \int_{0}^{1} d v \mu^{2} v^{2} \\
& \times\left[\left.(2 v)^{2(a-1)}(2 u v)^{2(b-1)} u A_{-a+1}(2 v \mu) \frac{d^{b-1}}{d\left(x^{2}\right)^{b-1}}\left(x^{-1} A_{1}(x)\right)\right|_{x=2 u v \mu}\right. \\
& \text { - } \left.\left.(2 v)^{2(b-1)}(2 u v)^{2(a-1)} A_{-a+1}(2 u v \mu) \frac{d^{b-1}}{d\left(x^{2}\right)^{b-1}}\left(x^{-1} A_{1}(x)\right)\right|_{x=2 v \mu}\right],
\end{aligned}
$$

$$
\begin{aligned}
& D_{+-}\left(\zeta, \mu_{a}\right) \rightarrow D_{+-}^{(0, a-1)}(\zeta, \mu) \\
&=-2 \int_{0}^{1} d u \int_{0}^{1} d v \sqrt{\zeta \mu} v^{3} u \\
& \times\left[\left.(2 u v)^{2(a-1)} J_{1}(2 v \zeta) \frac{d^{a-1}}{d\left(x^{2}\right)^{a-1}}\left(x^{-1} A_{1}(x)\right)\right|_{x=2 u v \mu}\right. \\
&\left.-\left.(2 v)^{2(a-1)} J_{1}(2 u v \zeta) \frac{d^{a-1}}{d\left(x^{2}\right)^{a-1}}\left(x^{-1} A_{1}(x)\right)\right|_{x=2 v \mu}\right], \\
& D_{-+}\left(\mu_{a}, \zeta^{\prime}\right) \rightarrow-D_{-+}^{(0, a-1)}\left(\zeta^{\prime}, \mu\right),
\end{aligned}
$$




$$
\begin{array}{rl}
D_{--}\left(\mu_{a}, \mu_{b}\right) \rightarrow D_{--}^{(a-1, b-1)}(\zeta, \mu) & \\
=2 \int_{0}^{1} & d u \int_{0}^{1} d v \mu v^{3} u \\
\times & {\left[\left.(2 v)^{2(a-1)}(2 u v)^{2(b-1)} A_{2-a}(2 v \mu) \frac{d^{b-1}}{d\left(x^{2}\right)^{b-1}}\left(x^{-1} A_{1}(x)\right)\right|_{x=2 u v \mu}\right.} \\
& \left.-\left.(2 u v)^{2(a-1)}(2 v)^{2(b-1)} A_{2-a}(2 u v \mu) \frac{d^{b-1}}{d\left(x^{2}\right)^{b-1}}\left(x^{-1} A_{1}(x)\right)\right|_{x=2 v \mu}\right],
\end{array}
$$

$$
\begin{aligned}
I_{+-}\left(\zeta, \mu_{a}\right) \rightarrow & I_{+-}^{(0, a-1)}(\zeta, \mu) \\
= & 2 \int_{0}^{1} d u \int_{0}^{1} d v \sqrt{\zeta \mu} \zeta \mu v\left[(2 u v)^{2(a-1)} J_{0}(2 v \zeta) A_{1-a}(2 u v \mu)\right. \\
& \left.\quad-(2 v)^{2(a-1)} J_{0}(2 u v \zeta) A_{1-a}(2 v \mu)\right], \\
I_{-+}\left(\mu, \zeta^{\prime}\right) \rightarrow & -I_{+-}^{(0, a-1)}\left(\zeta^{\prime}, \mu\right) \\
I_{--}\left(\mu_{a}, \mu_{b}\right) \rightarrow & I_{--}^{(a-1, b-1)}(\mu, \mu) \\
= & -2 \int_{0}^{1} d u \int_{0}^{1} d v \mu^{3} v\left[(2 v)^{2(a-1)}(2 u v)^{2(b-1)} A_{1-a}(2 v \mu) A_{1-b}(2 u v \mu)\right. \\
& \left.\quad-(2 u v)^{2(a-1)}(2 v)^{2(b-1)} A_{1-a}(2 u v \mu) A_{1-b}(2 v \mu)\right],
\end{aligned}
$$

where

$$
A_{k}(x)=x^{k} I_{k}(x), \quad 2 \frac{d I_{k}(x)}{d x}=x^{-1} I_{k-1}(x)-k x^{-2} I_{k}(x) .
$$

\section{Janossy density}

\section{C.1 Janossy density for the determinantal random point process}

Below we shall overview the definition of the Janossy density for the determinantal random point process [70-72]. Consider an ensemble of $N$ particles on $\mathbb{Z}$ with the joint distribution (see (1) in figure 11) given by

$$
p\left(n_{1}, \ldots, n_{N}\right)=\frac{1}{N !} \operatorname{det}\left[K\left(n_{i}, n_{j}\right)\right]_{i, j=1}^{N}, \quad n_{i} \in \mathbb{Z},
$$

with the kernel $\mathbf{K}=[K(n, m)]_{n, m \in \mathbb{Z}}$ obeying the projective condition:

$$
\mathbf{K} \cdot \mathbf{K}=\mathbf{K}, \quad \operatorname{tr} \mathbf{K}=N .
$$

Then the $k$-point function $R_{k}\left(n_{1}, \ldots, n_{k}\right)$ is given by

$$
R_{k}\left(n_{1}, \ldots, n_{k}\right)=\operatorname{det}\left[K\left(n_{i}, n_{j}\right)\right]_{i, j=1}^{k}
$$

Consider the probability $J_{k, I}\left(n_{1}, \ldots, n_{k}\right)$ of finding no particle in an interval $I \subset \mathbb{Z}$ except for $k$ designated point. (See (2) in figure 11.) $J_{k, I}\left(n_{1}, \ldots, n_{k}\right)$ is called Janossy 
$(1)$

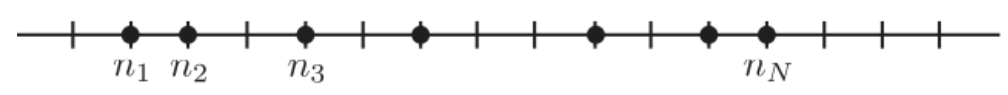

$(2)$

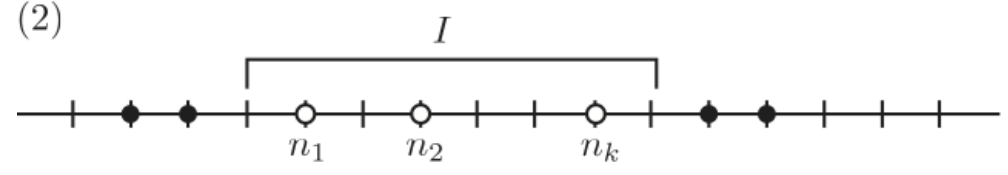

(3)

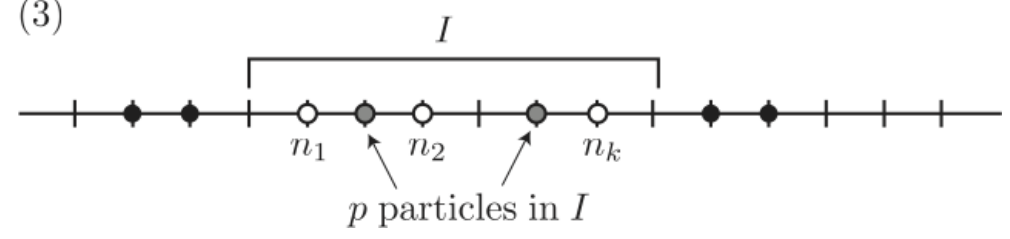

Figure 11. Distribution of particles. (1) there is a particle at each of the points $n_{i}(i=1, \ldots, N)$. (2) there are exactly $k$ particles in $I$, one in each of $k$ designated points $n_{i}(i=1, \ldots, k)$. (3) there are exactly $p$ particles in $I$ except for $k$ designated points $n_{i}(i=1, \ldots, k)$.

density [73], which is given by the restricted kernel $\mathbf{K}_{I}=[K(n, m)]_{n, m \in I}$ on $I$ for the determinantal point process.

$$
\begin{aligned}
J_{k, I}\left(n_{1}, \ldots, n_{k}\right) & =\operatorname{det}\left(\mathbb{I}-\mathbf{K}_{I}\right) \cdot \operatorname{det}\left[\left\langle n_{i}\left|\mathbf{K}_{I}\left(\mathbb{I}-\mathbf{K}_{I}\right)^{-1}\right| n_{j}\right\rangle\right]_{i, j=1}^{k} \\
& =(-1)^{k} \operatorname{det}\left|\begin{array}{cc}
-\left[\left\langle n_{i}\left|\mathbf{K}_{I}\right| n_{j}\right\rangle\right]_{i, j=1, \ldots, k} & -\left[\left\langle n\left|\mathbf{K}_{I}\right| n_{j}\right\rangle\right]_{j=1, \ldots, k ; n \in I} \\
-\left[\left\langle n_{i}\left|\mathbf{K}_{I}\right| m\right\rangle\right]_{i=1, \ldots, k ; m \in I} & {\left[\left\langle n\left|\left(\mathbb{I}-\mathbf{K}_{I}\right)\right| m\right\rangle\right]_{n, m \in I}}
\end{array}\right| .
\end{aligned}
$$

Here we denote the restricted kernel by $K_{I}(n, m)=\left\langle n\left|\mathbf{K}_{I}\right| m\right\rangle$ with the orthonormal complete basis $\{|n\rangle \mid n \in I\}$ and its dual $\{\langle n|| n \in I\}$. The first line of (C.4) is quoted e.g. from [74] $(\pi(X)$ on page 341$)$, and the second line is by the identity $\operatorname{det} D \cdot \operatorname{det}\left(A-C D^{-1} B\right)=\operatorname{det}|\underset{C}{A} \stackrel{B}{D}|$.

Generalization to the probability $J_{p, k, I}\left(n_{1}, \ldots, n_{k}\right)$ of finding exactly $p$ particles in $I$ except for $k$ designated points is straightforward (see (3) in figure 11). Just as in the case of the ordinary gap probability $(k=0)$, we merely introduce the spectral parameter $z$ so that $J_{p, k, I}\left(n_{1}, \ldots, n_{k}\right)$ is given by

$$
J_{p, k, I}\left(n_{1}, \ldots, n_{k}\right)=\left.\frac{1}{p !}\left(-\partial_{z}\right)^{p} \operatorname{det}\left(\mathbb{I}-z \mathbf{K}_{I}\right) \cdot \operatorname{det}\left[\left\langle n_{i}\left|\mathbf{K}_{I}\left(\mathbb{I}-z \mathbf{K}_{I}\right)^{-1}\right| n_{j}\right\rangle\right]_{i, j=1}^{k}\right|_{z=1} .
$$

For the continuous determinantal random point process on $X \subset \mathbb{R}$ with the measure $\mu$, the Janossy density $J_{k, I}\left(x_{1}, \ldots, x_{k}\right) \mu\left(d x_{1}\right) \cdots \mu\left(d x_{k}\right)$ for the distribution of the particles in a subset $I \subset X$ is defined as the probability density of finding exactly $k$ particles in $I$ and one at each of the $k$ infinitesimal intervals $\left(x_{i}, x_{i}+d x_{i}\right) \subset I . J_{k, I}\left(x_{1}, \ldots, x_{k}\right)$ is given by the Fredholm determinant $\operatorname{det}\left(\mathbb{I}-\mathbf{K}_{I}\right)$ and the determinant of $\mathbf{L}_{I}:=\mathbf{K}_{I}\left(\mathbb{I}-\mathbf{K}_{I}\right)^{-1}$ such that

$$
J_{k, I}\left(x_{1}, \ldots, x_{k}\right)=\operatorname{det}\left(\mathbb{I}-\mathbf{K}_{I}\right) \cdot \operatorname{det}\left[\mathbf{L}_{I}\left(x_{i}, x_{j}\right)\right]_{i, j=1}^{k}
$$


(A) $m_{a}^{2}<0$

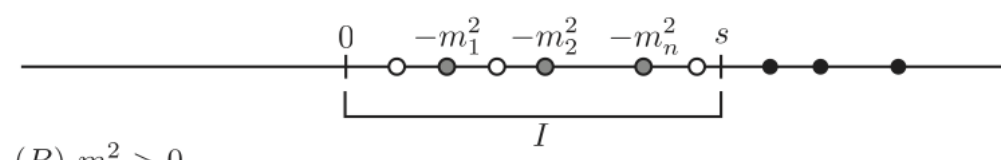

(B) $m_{a}^{2}>0$

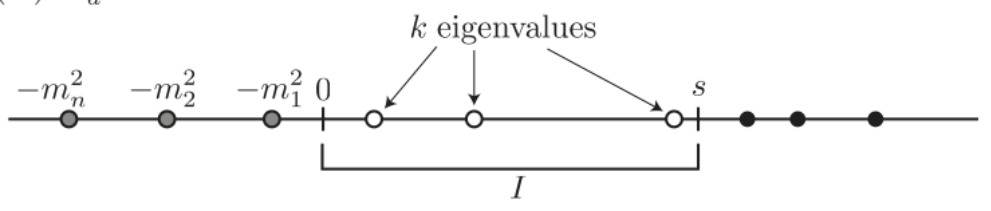

Figure 12. The determinantal random point process for $x_{i} i=1, \ldots, N+n$ with designated points at $-m_{a}^{2}(a=1, \ldots, n)$. (A) For $m_{a}^{2}<0$ : the Janossy density $J_{k, n,[0, s]}\left(\left\{-m_{a}^{2}\right\}\right)$. (B) For $m_{a}^{2}>0$ : the probability distribution $E\left(k ;[0, s] ;\left\{m_{a}\right\}\right)$ as an analytic continuation of the Janossy density.

\section{C.2 Massive chiral Gaussian ensemble with $N_{F}=\beta n$ fermions and the Janossy density}

Consider a block diagonal Hermitian matrix $H$ of Dyson index $\beta=1,2,4$ :

$$
H=\left(\begin{array}{cc}
0 & W \\
W^{\dagger} & 0
\end{array}\right), \quad W \in F^{N \times(N+\nu)}, \quad F=\mathbb{R}, \mathbb{C}, \mathbb{H} .
$$

The partition function $Z_{N, \beta, \nu}\left(\left\{m_{a}\right\}\right)$ of the massive chiral Gaussian ensemble with $N_{F}=\beta n$ fermions is given by

$$
\begin{aligned}
Z_{N, \beta, \nu}\left(\left\{m_{a}\right\}\right) & =\int d H \mathrm{e}^{-\beta \operatorname{tr} H^{2}} \prod_{a=1}^{n}\left(H+\mathrm{i} m_{a}\right)^{\beta} \\
& =\int_{0}^{\infty} \prod_{i=1}^{N}\left(d x_{i} x_{i}^{\frac{\beta(\nu+1)}{2}-1} \mathrm{e}^{-\beta x_{i}} \prod_{a=1}^{n}\left|x_{i}+m_{a}^{2}\right|^{\beta}\right) \prod_{i>j}^{N}\left|x_{i}-x_{j}\right|^{\beta} .
\end{aligned}
$$

It can further be rewritten as an $N+n$ eigenvalue integral in the following form (up to $m$-dependent prefactor $\left.C_{N, \beta, \nu}\left(\left\{m_{a}\right\}\right)\right)$ :

$$
\begin{aligned}
Z_{N, \beta, \nu}\left(\left\{m_{a}\right\}\right)= & \frac{1}{C_{N, \beta, \nu}\left(\left\{m_{a}\right\}\right)} \int_{-\infty}^{\infty} \prod_{i=1}^{N+n}\left(d x_{i} x_{i}^{\frac{\beta(\nu+1)}{2}-1} \mathrm{e}^{-\beta x_{i}}\right) \prod_{i>j}^{N+n}\left|x_{i}-x_{j}\right|^{\beta} \\
& \times \prod_{\ell=1}^{N} \theta\left(x_{\ell}\right) \prod_{k=N+1}^{N+n} \delta\left(x_{k}-\left(-m_{k-N}^{2}\right)\right)
\end{aligned}
$$

where $\theta(x)$ stands for the Heaviside function.

This partition function is regarded as that of the determinantal random point process for $x_{i}(i=1, \ldots, N+n)$ with designated points at $-m_{a}^{2}(a=1, \ldots, n)$. In the case of $m_{a}^{2}<0$, the Janossy density $J_{k, n,[0, s]}\left(-m_{1}^{2}, \ldots,-m_{n}^{2}\right)$ on the interval $I=[0, s](s>0)$ for the above massive chiral Gaussian ensemble is found by adopting the spectral kernel $K\left(z_{i}, z_{j}\right)[22-25]$ to eq. (C.6). (See (A) in figure 12.) 
Applying an analytic continuation with respect to the mass parameters $m_{a}$ 's, one finds the joint probability $E\left(k ;[0, s] ;\left\{m_{a}\right\}\right)$ in eq. (2.19) as the Janossy density $J_{k, n,[0, s]}\left(\left\{-m_{a}^{2}\right\}\right)$ with $m_{a}^{2}>0$. (See (B) in figure 12.)

\section{Probability distribution of the $k^{\text {th }}$ smallest eigenvalue}

The probability $E_{k}(s)=E\left(k ;[0, s] ;\left\{-m_{a}^{2}\right\}\right)$ of finding exactly $k$ eigenvalues in the interval $[0, s]$ is given by the $k^{\text {th }}$ derivative of the Fredholm determinant $\tau\left(z ;[0, s] ;\left\{-m_{a}^{2}\right\}\right)$ by the parameter $z$ such that

$$
E_{k}(s)=\left.\frac{1}{k !}\left(-\partial_{z}\right)^{k} \tau\left(z ;[0, s] ;\left\{-y_{a}\right\}\right)\right|_{z=1} .
$$

The Fredholm determinant and Pfaffian in eqs. (2.27) and (2.31) are represented by

$$
\tau\left(z ;[0, s] ;\left\{-m_{a}^{2}\right\}\right)=\operatorname{det}\left|\begin{array}{cc}
-\kappa & -\sqrt{z} \boldsymbol{k}^{\mathrm{T}} \\
-\sqrt{z} \boldsymbol{k} & \hat{\mathbb{I}}-z \boldsymbol{K}
\end{array}\right| / \operatorname{det}(-\kappa),
$$

where det stands for determinant and quaternionic determinant for unitary and symplectic ensembles, respectively. The Taylor expansion of $\tau\left(z ;[0, s] ;\left\{-m_{a}^{2}\right\}\right)$ in eq. (D.2) around $z=$ 1 is found as combinations of the functional traces $T_{n}$ 's as follows. (The same expansions for the quenched $(\alpha=0)$ ensembles are given in [20].)

$$
\begin{aligned}
E_{0}(s)= & \tau\left(z=1 ;[0, s] ;\left\{-y_{a}\right\}\right), \quad E_{1}(s)=E_{0}(s) \bar{T}_{1}, \quad E_{2}(s)=\frac{E_{0}(s)}{2 !}\left(\bar{T}_{1}^{2}-\bar{T}_{2}\right), \\
E_{3}(s)= & \frac{E_{0}(s)}{3 !}\left(\bar{T}_{1}^{3}-3 \bar{T}_{1} \bar{T}_{2}+\bar{T}_{3}\right), \\
E_{4}(s)= & \frac{E_{0}(s)}{4 !}\left(\bar{T}_{1}^{4}-6 \bar{T}_{1}^{2} \bar{T}_{2}+3 \bar{T}_{2}^{2}+4 \bar{T}_{1} \bar{T}_{3}-\bar{T}_{4}\right), \\
E_{5}(s)= & \frac{E_{0}(s)}{5 !}\left(\bar{T}_{1}^{5}-10 \bar{T}_{1}^{3} \bar{T}_{2}+10 \bar{T}_{1}^{2} \bar{T}_{3}+15 \bar{T}_{1} \bar{T}_{2}^{2}-5 \bar{T}_{1} \bar{T}_{4}-10 \bar{T}_{2} \bar{T}_{3}+\bar{T}_{5}\right), \\
E_{6}(s)= & \frac{E_{0}(s)}{6 !}\left(\bar{T}_{1}^{6}-15 \bar{T}_{1}^{4} \bar{T}_{2}+20 \bar{T}_{1}^{3} \bar{T}_{3}+45 \bar{T}_{1}^{2} \bar{T}_{2}^{2}-15 \bar{T}_{1}^{2} \bar{T}_{4}-60 \bar{T}_{1} \bar{T}_{2} \bar{T}_{3}-15 \bar{T}_{2}^{3}\right. \\
\left.\quad+6 \bar{T}_{1} \bar{T}_{5}+15 \bar{T}_{2} \bar{T}_{4}+10 \bar{T}_{2}^{3}-\bar{T}_{6}\right), & (\mathrm{D} .3) \\
E_{7}(s)= & \frac{E_{0}(s)}{7 !}\left(\bar{T}_{1}^{7}-21 \bar{T}_{1}^{5} \bar{T}_{2}+35 \bar{T}_{1}^{4} \bar{T}_{3}+105 \bar{T}_{1}^{3} \bar{T}_{1}^{2}-35 \bar{T}_{1}^{3} \bar{T}_{4}-210 \bar{T}_{1}^{2} \bar{T}_{2} \bar{T}_{3}-105 \bar{T}_{1} \bar{T}_{2}^{3}\right. \\
\quad & \quad+21 \bar{T}_{1}^{2} \bar{T}_{5}+105 \bar{T}_{1} \bar{T}_{2} \bar{T}_{4}+70 \bar{T}_{1} \bar{T}_{3}^{2}+105 \bar{T}_{2}^{2} \bar{T}_{3}-7 \bar{T}_{1} \bar{T}_{6}-21 \bar{T}_{2} \bar{T}_{5} \\
& \left.\quad-35 \bar{T}_{3} \bar{T}_{4}+\bar{T}_{7}\right) .
\end{aligned}
$$

The functional traces consist of operators $\mathcal{K}^{(n)}$ 's are given by

$$
\begin{aligned}
& \mathcal{K}(z)=\left(\begin{array}{cc}
-\kappa & -\sqrt{z} \boldsymbol{k}^{\mathrm{T}} \\
-\sqrt{z} \boldsymbol{k} & \mathbb{I}-z \boldsymbol{K}
\end{array}\right), \quad \mathcal{K}^{(0)}=\mathcal{K}(z=1)=\left(\begin{array}{cc}
-\kappa & -\boldsymbol{k}^{\mathrm{T}} \\
-\boldsymbol{k} \mathbb{I}-\boldsymbol{K}
\end{array}\right), \\
& \mathcal{K}^{(1)}=-\left.\frac{\partial}{\partial z} \mathcal{K}(z)\right|_{z=1}=\left(\begin{array}{cc}
0 & \frac{1}{2} \boldsymbol{k}^{\mathrm{T}} \\
\frac{1}{2} \boldsymbol{k} & \boldsymbol{K}
\end{array}\right), \\
& \mathcal{K}^{(n)}=\left.\frac{\partial^{n}}{\partial z^{n}} \mathcal{K}(z)\right|_{z=1}=(-1)^{n-1} \frac{(2 n-3) !}{2^{n}}\left(\begin{array}{cc}
0 & \frac{1}{2} \boldsymbol{k}^{\mathrm{T}} \\
\frac{1}{2} \boldsymbol{k} & 0
\end{array}\right), \quad(n \geq 2) .
\end{aligned}
$$


Using these operators, one can show $\bar{T}_{n}(k=1, \ldots, 7)$ as follows.

$$
\begin{aligned}
& \bar{T}_{1}=\frac{1}{2} \operatorname{tr}\left[\mathcal{K}^{(1)} \cdot \mathcal{K}^{(0)-1}\right] \text {, } \\
& \bar{T}_{2}=\frac{1}{2} \operatorname{tr}\left[\left(\mathcal{K}^{(1)} \cdot \mathcal{K}^{(0)-1}\right)^{2}\right]+\frac{1}{2} \operatorname{tr}\left[\mathcal{K}^{(2)} \cdot \mathcal{K}^{(0)-1}\right], \\
& \bar{T}_{3}=2 ! \cdot \frac{1}{2} \operatorname{tr}\left[\left(\mathcal{K}^{(1)} \cdot \mathcal{K}^{(0)-1}\right)^{3}\right]+3 \cdot \frac{1}{2} \operatorname{tr}\left[\mathcal{K}^{(1)} \cdot \mathcal{K}^{(0)-1} \cdot \mathcal{K}^{(2)} \cdot \mathcal{K}^{(0)-1}\right] \\
& +\frac{1}{2} \operatorname{tr}\left[\mathcal{K}^{(3)} \cdot \mathcal{K}^{(0)-1}\right] \text {, } \\
& \bar{T}_{4}=3 ! \cdot \frac{1}{2} \operatorname{tr}\left[\left(\mathcal{K}^{(1)} \cdot \mathcal{K}^{(0)-1}\right)^{4}\right]+12 \cdot \frac{1}{2} \operatorname{tr}\left[\left(\mathcal{K}^{(1)} \cdot \mathcal{K}^{(0)-1}\right)^{2} \cdot \mathcal{K}^{(2)} \cdot \mathcal{K}^{(0)-1}\right] \\
& +3 \cdot \frac{1}{2} \operatorname{tr}\left[\left(\mathcal{K}^{(2)} \cdot \mathcal{K}^{(0)-1}\right)^{2}\right]+4 \cdot \frac{1}{2} \operatorname{tr}\left[\mathcal{K}^{(1)} \cdot \mathcal{K}^{(0)-1} \cdot \mathcal{K}^{(3)} \cdot \mathcal{K}^{(0)-1}\right] \\
& +\frac{1}{2} \operatorname{tr}\left[\mathcal{K}^{(4)} \cdot \mathcal{K}^{(0)-1}\right] \text {, } \\
& \bar{T}_{5}=4 ! \cdot \frac{1}{2} \operatorname{tr}\left[\left(\mathcal{K}^{(1)} \cdot \mathcal{K}^{(0)-1}\right)^{5}\right]+60 \cdot \frac{1}{2} \operatorname{tr}\left[\left(\mathcal{K}^{(1)} \cdot \mathcal{K}^{(0)-1}\right)^{3} \cdot \mathcal{K}^{(2)} \cdot \mathcal{K}^{(0)-1}\right] \\
& +20 \cdot \frac{1}{2} \operatorname{tr}\left[\left(\mathcal{K}^{(1)} \cdot \mathcal{K}^{(0)-1}\right)^{2} \cdot \mathcal{K}^{(3)} \cdot \mathcal{K}^{(0)-1}\right] \\
& +30 \cdot \frac{1}{2} \operatorname{tr}\left[\mathcal{K}^{(1)} \cdot \mathcal{K}^{(0)-1} \cdot\left(\mathcal{K}^{(2)} \cdot \mathcal{K}^{(0)-1}\right)^{2}\right]+5 \cdot \frac{1}{2} \operatorname{tr}\left[\mathcal{K}^{(1)} \cdot \mathcal{K}^{(0)-1} \cdot \mathcal{K}^{(4)} \cdot \mathcal{K}^{(0)-1}\right] \\
& +10 \cdot \frac{1}{2} \operatorname{tr}\left[\mathcal{K}^{(2)} \cdot \mathcal{K}^{(0)-1} \cdot \mathcal{K}^{(3)} \cdot \mathcal{K}^{(0)-1}\right]+\frac{1}{2} \operatorname{tr}\left[\mathcal{K}^{(5)} \cdot \mathcal{K}^{(0)-1}\right], \\
& \bar{T}_{6}=5 ! \cdot \frac{1}{2} \operatorname{tr}\left[\left(\mathcal{K}^{(1)} \cdot \mathcal{K}^{(0)-1}\right)^{6}\right]+360 \cdot \frac{1}{2} \operatorname{tr}\left[\left(\mathcal{K}^{(1)} \cdot \mathcal{K}^{(0)-1}\right)^{4} \cdot \mathcal{K}^{(2)} \cdot \mathcal{K}^{(0)-1}\right] \\
& +120 \cdot \frac{1}{2} \operatorname{tr}\left[\left(\mathcal{K}^{(1)} \cdot \mathcal{K}^{(0)-1}\right)^{3} \cdot \mathcal{K}^{(3)} \cdot \mathcal{K}^{(0)-1}\right] \\
& +180 \cdot \frac{1}{2} \operatorname{tr}\left[\left(\mathcal{K}^{(1)} \cdot \mathcal{K}^{(0)-1}\right)^{2} \cdot\left(\mathcal{K}^{(2)} \cdot \mathcal{K}^{(0)-1}\right)^{2}\right] \\
& +90 \cdot \frac{1}{2} \operatorname{tr}\left[\mathcal{K}^{(1)} \cdot \mathcal{K}^{(0)-1} \cdot \mathcal{K}^{(2)} \cdot \mathcal{K}^{(0)-1} \cdot \mathcal{K}^{(1)} \cdot \mathcal{K}^{(0)-1} \cdot \mathcal{K}^{(2)} \cdot \mathcal{K}^{(0)-1}\right] \\
& +30 \cdot \frac{1}{2} \operatorname{tr}\left[\left(\mathcal{K}^{(1)} \cdot \mathcal{K}^{(0)-1}\right)^{2} \cdot \mathcal{K}^{(4)} \cdot \mathcal{K}^{(0)-1}\right] \\
& +120 \cdot \frac{1}{2} \operatorname{tr}\left[\mathcal{K}^{(1)} \cdot \mathcal{K}^{(0)-1} \cdot \mathcal{K}^{(2)} \cdot \mathcal{K}^{(0)-1} \cdot \mathcal{K}^{(3)} \cdot \mathcal{K}^{(0)-1}\right] \\
& +30 \cdot \frac{1}{2} \operatorname{tr}\left[\left(\mathcal{K}^{(2)} \cdot \mathcal{K}^{(0)-1}\right)^{3}\right] \\
& +6 \cdot \frac{1}{2} \operatorname{tr}\left[\mathcal{K}^{(1)} \cdot \mathcal{K}^{(0)-1} \cdot \mathcal{K}^{(5)} \cdot \mathcal{K}^{(0)-1}\right]+15 \cdot \frac{1}{2} \operatorname{tr}\left[\mathcal{K}^{(2)} \cdot \mathcal{K}^{(0)-1} \cdot \mathcal{K}^{(4)} \cdot \mathcal{K}^{(0)-1}\right] \\
& +10 \cdot \frac{1}{2} \operatorname{tr}\left[\left(\mathcal{K}^{(3)} \cdot \mathcal{K}^{(0)-1}\right)^{2}\right]+\frac{1}{2} \operatorname{tr}\left[\mathcal{K}^{(6)} \cdot \mathcal{K}^{(0)-1}\right], \\
& \bar{T}_{7}=6 ! \cdot \frac{1}{2} \operatorname{tr}\left[\left(\mathcal{K}^{(1)} \cdot \mathcal{K}^{(0)-1}\right)^{7}\right]+2520 \cdot \frac{1}{2} \operatorname{tr}\left[\left(\mathcal{K}^{(1)} \cdot \mathcal{K}^{(0)-1}\right)^{5} \cdot \mathcal{K}^{(2)} \cdot \mathcal{K}^{(0)-1}\right] \\
& +840 \cdot \frac{1}{2} \operatorname{tr}\left[\left(\mathcal{K}^{(1)} \cdot \mathcal{K}^{(0)-1}\right)^{4} \cdot \mathcal{K}^{(3)} \cdot \mathcal{K}^{(0)-1}\right]
\end{aligned}
$$




$$
\begin{aligned}
& +1260 \cdot \frac{1}{2} \operatorname{tr}\left[\left(\mathcal{K}^{(1)} \cdot \mathcal{K}^{(0)-1}\right)^{3} \cdot\left(\mathcal{K}^{(2)} \cdot \mathcal{K}^{(0)-1}\right)^{2}\right] \\
& +1260 \cdot \frac{1}{2} \operatorname{tr}\left[\left(\mathcal{K}^{(1)} \cdot \mathcal{K}^{(0)-1}\right)^{2} \cdot \mathcal{K}^{(2)} \cdot \mathcal{K}^{(0)-1} \cdot \mathcal{K}^{(1)} \cdot \mathcal{K}^{(0)-1} \cdot \mathcal{K}^{(2)} \cdot \mathcal{K}^{(0)-1}\right] \\
& +210 \cdot \frac{1}{2} \operatorname{tr}\left[\left(\mathcal{K}^{(1)} \cdot \mathcal{K}^{(0)-1}\right)^{3} \cdot \mathcal{K}^{(4)} \cdot \mathcal{K}^{(0)-1}\right] \\
& +840 \cdot \frac{1}{2} \operatorname{tr}\left[\left(\mathcal{K}^{(1)} \cdot \mathcal{K}^{(0)-1}\right)^{2} \cdot \mathcal{K}^{(2)} \cdot \mathcal{K}^{(0)-1} \cdot \mathcal{K}^{(3)} \cdot \mathcal{K}^{(0)-1}\right] \\
& +420 \cdot \frac{1}{2} \operatorname{tr}\left[\mathcal{K}^{(1)} \cdot \mathcal{K}^{(0)-1} \cdot \mathcal{K}^{(2)} \cdot \mathcal{K}^{(0)-1} \cdot \mathcal{K}^{(1)} \cdot \mathcal{K}^{(0)-1} \cdot \mathcal{K}^{(3)} \cdot \mathcal{K}^{(0)-1}\right] \\
& +630 \cdot \frac{1}{2} \operatorname{tr}\left[\mathcal{K}^{(1)} \cdot \mathcal{K}^{(0)-1} \cdot\left(\mathcal{K}^{(2)} \cdot \mathcal{K}^{(0)-1}\right)^{3}\right] \\
& +42 \cdot \frac{1}{2} \operatorname{tr}\left[\left(\mathcal{K}^{(1)} \cdot \mathcal{K}^{(0)-1}\right)^{2} \cdot \mathcal{K}^{(5)} \cdot \mathcal{K}^{(0)-1}\right] \\
& +210 \cdot \frac{1}{2} \operatorname{tr}\left[\mathcal{K}^{(1)} \cdot \mathcal{K}^{(0)-1} \cdot \mathcal{K}^{(2)} \cdot \mathcal{K}^{(0)-1} \mathcal{K}^{(4)} \cdot \mathcal{K}^{(0)-1}\right] \\
& +140 \cdot \frac{1}{2} \operatorname{tr}\left[\mathcal{K}^{(1)} \cdot \mathcal{K}^{(0)-1} \cdot\left(\mathcal{K}^{(3)} \cdot \mathcal{K}^{(0)-1}\right)^{2}\right] \\
& +210 \cdot \frac{1}{2} \operatorname{tr}\left[\left(\mathcal{K}^{(2)} \cdot \mathcal{K}^{(0)-1}\right)^{2} \cdot \mathcal{K}^{(3)} \cdot \mathcal{K}^{(0)-1}\right] \\
& +7 \cdot \frac{1}{2} \operatorname{tr}\left[\mathcal{K}^{(1)} \cdot \mathcal{K}^{(0)-1} \cdot \mathcal{K}^{(6)} \cdot \mathcal{K}^{(0)-1}\right] \\
& +21 \cdot \frac{1}{2} \operatorname{tr}\left[\mathcal{K}^{(2)} \cdot \mathcal{K}^{(0)-1} \cdot \mathcal{K}^{(5)} \cdot \mathcal{K}^{(0)-1}\right] \\
& +\frac{1}{2} \operatorname{tr}\left[\mathcal{K}^{(7)} \cdot \mathcal{K}^{(0)-1}\right] \text {. }
\end{aligned}
$$

The above expansion of the functional trace can also be considered as follows. Rewriting the Fredholm determinant and Pfaffian given in eq. (D.2) into the following form: ${ }^{9}$

$$
\operatorname{det}\left|\begin{array}{cc}
-\kappa & -\sqrt{z} \boldsymbol{k}^{\mathrm{T}} \\
-\sqrt{z} \boldsymbol{k} & \hat{\mathbb{I}}-z \boldsymbol{K}
\end{array}\right| / \operatorname{det}(-\kappa)=\operatorname{det}\left(\hat{\mathbb{I}}-z\left(\boldsymbol{K}-\boldsymbol{k} \kappa^{-1} \boldsymbol{k}^{\mathrm{T}}\right)\right),
$$

then one finds that $E_{k}(s)$ 's in eq. (D.2) are represented as the quenched model. Using the representation eq. (2.6) in [20], we obtain a little different expansion with the functional traces of the resolvents $T_{n}(s)=\operatorname{tr}\left(\tilde{\boldsymbol{K}}(\mathbb{I}-\tilde{\boldsymbol{K}})^{-1}\right)^{n}$ for the Fredholm determinant and $T_{n}(s)=$ $\operatorname{tr}\left(\tilde{\boldsymbol{K}}(\mathbb{I}-\tilde{\boldsymbol{K}})^{-1}\right)^{n} / 2$ for the Fredholm Pfaffian with $\tilde{\boldsymbol{K}}=\boldsymbol{K}-\boldsymbol{k} \kappa^{-1} \boldsymbol{k}^{\mathrm{T}}$.

\section{E Gauss-Legendre quadrature rule}

The quadrature rule is an efficient method to perform the numerical evaluation for the integral of the smooth function. The quadrature formula for the integral over the interval is represented as [49]

$$
Q_{I}(f)=\sum_{i=1}^{m} w_{i} f\left(x_{i}\right) \approx \int_{I} f(x) d x
$$

\footnotetext{
${ }^{9}$ The authors thank the anonymous referee for pointing out such expansion.
} 


\begin{tabular}{|c|c|c|}
\hline$M$ & $x_{i}^{[-1,1]}$ & $w_{i}^{[-1,1]}$ \\
\hline 1 & 0 & 2 \\
\hline 2 & $\pm \sqrt{1 / 3}$ & 1 \\
\hline \multirow{2}{*}{3} & 0 & $8 / 9$ \\
\cline { 2 - 3 } & $\pm \sqrt{3 / 5}$ & $5 / 9$ \\
\hline \multirow{2}{*}{4} & $\pm \sqrt{(3-2 \sqrt{6 / 5}) / 7}$ & $\frac{18+\sqrt{30}}{36}$ \\
\cline { 2 - 3 } & $\pm \sqrt{(3+2 \sqrt{6 / 5}) / 7}$ & $\frac{18+\sqrt{30}}{36}$ \\
\hline \multirow{2}{*}{5} & 0 & $128 / 225$ \\
\cline { 2 - 3 } & $\pm \frac{1}{3} \sqrt{5-2 \sqrt{10 / 7}}$ & $\frac{322+13 \sqrt{70}}{900}$ \\
\cline { 2 - 3 } & $\pm \frac{1}{3} \sqrt{5+2 \sqrt{10 / 7}}$ & $\frac{322-13 \sqrt{70}}{900}$ \\
\hline
\end{tabular}

Table 1. Nodes and weights of the Gauss-Legendre rule.

where $w_{i}$ and $x_{i}$ denote the weight and nodes, respectively, determined by the prescription of the quadrature rule. There are several kinds of quadrature rules. The most basic method is the Gauss-Legendre rule and more efficient one is the Clenshaw-Curtis rule. In the following, we will summarize the Gauss-Legendre rule.

Let $I=[-1,1]$ and $M \in \mathbb{N}$.

1. The node $x_{i}^{[-1,1]}$ is given by the $i^{\text {th }}$ zero of the Legendre polynomial $P_{M}(x)$.

2. The weight $w_{i}^{[-1,1]}$ is given by

$$
w_{i}^{[-1,1]}=\frac{2}{\left(1-x_{i}^{2}\right)^{2} P_{M}^{\prime}\left(x_{i}\right)^{2}} .
$$

For some lower orders $M$, nodes and weights are listed in the following table [49].

The following proposition holds for the Gauss-Legendre quadrature rule.

Proposition E.1. The Gauss-Legendre quadrature rule of order $M$ is exact, if $f(x)$ is an $(2 M-1)^{\text {th }}$ order (or lesser) polynomial of $x$.

By a simple change of variable, one finds the quadrature formula for the interval $I=[a, b]$.

$$
\begin{aligned}
\int_{a}^{b} d x f(x) & =\frac{b-a}{2} \int_{-1}^{1} f\left(\frac{b-a}{2} x+\frac{a+b}{2}\right) \\
& \approx \frac{b-a}{2} \sum_{i=1}^{M} w_{i}^{[-1,1]} f\left(\frac{b-a}{2} x_{i}^{[-1,1]}+\frac{a+b}{2}\right) .
\end{aligned}
$$

In particular for $I=[0, s]$, the quadrature formula reduces to

$$
\int_{0}^{s} d x f(x) \approx \sum_{i=1}^{M} \frac{s w_{i}}{2} f\left(\frac{s}{2}\left(x_{i}+1\right)\right) .
$$




\begin{tabular}{|cc|crrr|}
\hline lattice size & $\beta$ & $\hat{\Sigma}$ & $\mu$ & $\chi^{2} /$ d.o.f & num $(\nu=0)$ \\
\hline $8^{4}$ & 1.100 & $0.2176(27)$ & $8.91(11)$ & $0.30(17)$ & 230 \\
& 1.200 & $0.1997(24)$ & $8.18(10)$ & $0.25(17)$ & 260 \\
& 1.300 & $0.1651(14)$ & $6.76(05)$ & $0.45(20)$ & 314 \\
& 1.350 & $0.1378(12)$ & $5.65(05)$ & $0.27(16)$ & 467 \\
& 1.375 & $0.1238(13)$ & $5.07(05)$ & $0.52(20)$ & 407 \\
& 1.400 & $0.0781(11)$ & $3.20(04)$ & $11.00(93)$ & 843 \\
& 1.425 & $0.0215(01)$ & $0.880(4)$ & $2.85(47)$ & 2338 \\
\hline $12^{4}$ & 1.100 & $0.1903(23)$ & $39.46(48)$ & $0.66(25)$ & 399 \\
& 1.300 & $0.1425(44)$ & $29.54(91)$ & $0.20(15)$ & 63 \\
& 1.350 & $0.1263(23)$ & $26.19(49)$ & $0.37(20)$ & 38 \\
& 1.375 & $0.1156(39)$ & $23.98(81)$ & $0.13(13)$ & 45 \\
& 1.400 & $0.0831(14)$ & $17.23(29)$ & $0.50(23)$ & 106 \\
& 1.425 & $0.0598(10)$ & $12.41(21)$ & $0.37(20)$ & 206 \\
& 1.450 & $0.0209(04)$ & $4.32(08)$ & $6.74(73)$ & 600 \\
\hline $16^{4}$ & 1.350 & $0.1252(20)$ & $82.0(1.3)$ & $0.34(16)$ & 105 \\
& 1.375 & $0.1064(34)$ & $69.8(2.3)$ & $0.22(16)$ & 41 \\
& 1.400 & $0.0799(12)$ & $52.35(80)$ & $0.37(21)$ & 155 \\
& 1.425 & $0.0521(05)$ & $34.13(33)$ & $0.48(20)$ & 369 \\
& 1.450 & $0.0246(02)$ & $16.14(17)$ & $0.83(26)$ & 561 \\
1.475 & $0.0083(01)$ & $5.47(12)$ & $1.32(30)$ & 248 \\
\hline
\end{tabular}

Table 2. Fit result of $\hat{\Sigma}$, chiral condensate in the lattice unit, together with the corresponding value of $\mu$. The bare coupling constant is given through $\beta=4 / g^{2}$. The most right column is the number of configurations we used in each of the fitting, which belong to the topological charge $\nu=0$ sector. The reduced chi squared, $\chi^{2} /$ d.o.f, indicates the quality of the fitting.

In particular for the numerical evaluation of the Fredholm determinant on $\tau\left(z ;[0, s] ;\left\{\mu_{a}\right\}\right)$, the nodes and weights for $I=[0, s]$ are chosen as

$$
\zeta_{i}=\frac{s}{2}\left(x_{i}^{[-1,1]}+1\right), \quad w_{i}=\frac{s w_{i}^{[-1,1]}}{2} .
$$

\section{F Details of the lattice result}

In table 2, we list the result of the fitting of lattice data.

\section{G Estimation of the correlation matrix}

An element of the correlation matrix is given

$$
C_{i j}=\left\langle\left(y_{i}-\left\langle y_{i}\right\rangle\right)\left(y_{j}-\left\langle y_{j}\right\rangle\right)\right\rangle,
$$

where $y_{i}=I\left(\hat{s}_{i}\right)$ with $I\left(\hat{s}_{i}\right)=\int_{0}^{\hat{s}} d \hat{\lambda}_{1} p_{1}^{\text {latt. }}\left(\hat{\lambda}_{1} ; \hat{m}_{f}\right)$ defined in eq. (4.4) and $\hat{s}_{i}$ is the upper end of the $i$-th bin. The bracket $\langle\cdot\rangle$ represents the average over lattice configurations which 
belong to $\nu=0$ sector. Since the correlation matrix is an average of fluctuation, one needs to use a resampling method like jackknife or bootstrapping to estimate. In this analysis, we use the jackknife method.

What we need in the fitting is not the correlation matrix itself but its inverse. As the estimate of $C$ contains some error, we need some care to invert it. If the bin width is too fine, neighboring bins may give (almost) the same value which causes zero-mode (or almost zero-mode) of the correlation matrix. If eigenvalue of $C$ is too small, the relative error of the eigenvalue becomes large, which makes estimation of $C^{-1}$ unreliable. Note that the smallest eigenmode gives the largest contribution to the inverse.

We therefore employ the following steps. First of all, some of the bins do not have eigenvalues of the Dirac operator in it (the largest several bins and sometimes the first bin(s)). Let us suppose that $i$-th bin has no eigenvalue. Then, $i$-th column/row of the correlation matrix, $C_{i j}$ and $C_{j i}$ for arbitrary $j$ becomes zero as $y_{i}$ is always 1 (or always 0 ). This obviously reduces the rank of $C$. We therefore replace the diagonal element $C_{i i}=0$ with the upper bound of the estimate, $1 / n^{3}$, where $n$ is number of independent configurations we use. ${ }^{10}$ The off-diagonal elements are kept zero. After this modification of the correlation matrix, which is now denoted as $C^{\prime}$, we still may have very small eigenvalues. Numerically, we even may observe (small) negative eigenvalue of $C^{\prime}{ }^{11}$ We therefore truncate the correlation matrix by cutting small eigenmodes in inverting the matrix to give an improved estimate of the inverse of the correlation matrix $C_{\mathrm{imp}}^{-1}$. The cutoff $c_{\text {cut }}$ we use is 0.1 times smallest diagonal element, $c_{\text {cut }}=0.1 / n^{3}$. That is,

$$
C^{\prime}|i\rangle=c_{i}|i\rangle, \quad C_{\text {imp. }}^{-1}=\sum_{i \text { s.t. } c_{i}>c_{\text {cut }}}|i\rangle \frac{1}{c_{i}}\langle i| .
$$

\section{H Hybrid Monte Carlo (HMC) for RMT}

A hybrid Monte Carlo simulation technique [75] is applicable to finite $N$ random matrix theory.

By introducing $\zeta_{i}=\sqrt{8 N x_{i}}$ and $\mu_{a}=\sqrt{8 N} m_{a}$ as eq. (2.9), the partition function $(2.2)$ becomes

$$
Z=C \int_{0}^{\infty} \cdots \int_{0}^{\infty} \prod_{i=1}^{N} d \zeta_{i} e^{-S}
$$

where $C$ represents irrelevant normalization factor and the action is

$$
S=\sum_{i=1}^{N}\left(\beta \frac{\zeta_{i}^{2}}{8 N}-\frac{\beta(\nu+1)-1}{2} \ln \left(\zeta_{i}^{2}\right)-\sum_{a=1}^{n_{f}} \ln \left(\zeta_{i}^{2}+\mu_{a}^{2}\right)-\beta \sum_{j<i} \ln \left|\zeta_{i}^{2}-\zeta_{j}^{2}\right|\right) .
$$

The dynamical variables here is the eigenvalue $\zeta_{i}$. The Hamiltonian for the HMC is

$$
H=\sum_{i=1}^{N} \frac{p_{i}^{2}}{2}+S
$$

\footnotetext{
${ }^{10}$ This value is estimated by assuming that 1 configuration has 1 eigenvalue in the bin, and other $n-1$ configurations do not have any. We also assume that $n$ is large enough.

${ }^{11}$ The correlation matrix must be positive semi-definite, but with finite statistics and numerical precision, we may observe negative eigenvalue.
} 


\begin{tabular}{|c|rrr|}
\hline$N$ & $N_{f}=2$ & $N_{f}=4$ & $N_{f}=8$ \\
\hline 250 & 2495000 & 1535950 & \\
1000 & 245000 & 495000 & 245000 \\
2000 & 245000 & 245000 & 245000 \\
4000 & & & 145000 \\
\hline
\end{tabular}

Table 3. Number of independent Monte Carlo configurations used in section 3.

where $p_{i}$ the conjugate momentum to $\zeta_{i}$. It is straightforward to write down the equation of motions and apply the HMC algorithm. For the molecular dynamical time evolution, we use a leapfrog integrator.

The only non-trivial part is ordering of the variables. We assume that $0<\zeta_{1}<\zeta_{2}<$ $\cdots<\zeta_{N}$. Since there is a divergence in the potential at $\zeta_{i}=0$ and $\zeta_{i}=\zeta_{j}(i \neq j)$, if the initial configuration satisfies this ordering, a smooth molecular dynamical evolution keeps the configuration satisfy the same constraint. Discrete time evolutions, however, can break the constraint so that we use the so called retry trick. We check whether the trial configuration satisfies the constraint before the metropolis test. If it does not, rerun the molecular dynamics with the same random momentum but a finer time step, $\delta \tau \rightarrow \delta \tau / 2$. If the constraint is still broken after several reductions of the time step (our limit is 6 times), the trial configuration is rejected. For $\beta=4$, the frequency of the retry is order $0.01 \%$ and we did not encounter rejections for this reason. As $\beta$ becomes smaller, the effect of the potential barrier becomes weaker. In fact, more frequent retries are needed for $\beta=2$, and some trial configurations are rejected in the end. Note that $\beta=1$ and $\nu=0$, the potential barrier at $\zeta_{i}=0$ disappears.

Here is some parameters we used in $\beta=4$ case. The trajectory length between Metropolis test is $\tau=1$. We keep the acceptance ratio rather high, typically $0.96-0.97$, to reduce the frequency of retries. To avoid the auto correlation, we measure the smallest $10 \zeta_{i}$ every 10 trajectories and all $\zeta_{i}$ every 500 trajectories. In making the distribution in figures $3,4,6-8$, we check the integrated auto correlation, which is $2 \tau_{\text {int }} \lesssim 1.2$ and used every 2 measurements.

The number of independent configurations used to plot figures $3,4,6-8$ in section 3 are tabulated in table 3 .

\section{Data of $k^{\text {th }}$ smallest eigenvalue distributions for chiral GSE with $N_{F}=8$}

Numerical data of $F_{k}(s ; \mu)(k=1,2,3,4)$ for the chiral GSE with $N_{F}=8$ degenerate flavors, in the range $0 \leq s \leq 20$ and $0 \leq \mu \leq 100$ are provided as supplementary material.

Open Access. This article is distributed under the terms of the Creative Commons Attribution License (CC-BY 4.0), which permits any use, distribution and reproduction in any medium, provided the original author(s) and source are credited. 


\section{References}

[1] M. Berry and J.P. Keating, The Riemann zeros and eigenvalue asymptotics, SIAM Rev. 41 (2012) 236.

[2] R.G. Edwards, U.M. Heller, J.E. Kiskis and R. Narayanan, Quark spectra, topology and random matrix theory, Phys. Rev. Lett. 82 (1999) 4188 [hep-th/9902117] [INSPIRE].

[3] T.A. DeGrand and S. Schaefer, Chiral properties of two-flavor QCD in small volume and at large lattice spacing, Phys. Rev. D 72 (2005) 054503 [hep-lat/0506021] [InSPIRE].

[4] JLQCD collaboration, Two-flavor lattice QCD simulation in the $\epsilon$-regime with exact chiral symmetry, Phys. Rev. Lett. 98 (2007) 172001 [hep-lat/0702003] [INSPIRE].

[5] P.V. Buividovich, E.V. Luschevskaya and M.I. Polikarpov, Finite-temperature chiral condensate and low-lying Dirac eigenvalues in quenched SU(2) lattice gauge theory, Phys. Rev. D 78 (2008) 074505 [arXiv:0809.3075] [INSPIRE].

[6] C. Lehner, J. Bloch, S. Hashimoto and T. Wettig, Geometry dependence of RMT-based methods to extract the low-energy constants Sigma and F, JHEP 05 (2011) 115 [arXiv:1101.5576] [INSPIRE].

[7] E.V. Shuryak and J.J.M. Verbaarschot, Random matrix theory and spectral sum rules for the Dirac operator in QCD, Nucl. Phys. A 560 (1993) 306 [hep-th/9212088] [INSPIRE].

[8] P.H. Damgaard, U.M. Heller, R. Niclasen and K. Rummukainen, Low lying eigenvalues of the QCD Dirac operator at finite temperature, Nucl. Phys. B 583 (2000) 347 [hep-lat/0003021] [INSPIRE].

[9] T.W. Appelquist, D. Karabali and L.C.R. Wijewardhana, Chiral hierarchies and the flavor changing neutral current problem in technicolor, Phys. Rev. Lett. 57 (1986) 957 [INSPIRE].

[10] C. Pica, Beyond the standard model: charting fundamental interactions via lattice simulations, PoS(LATTICE 2016)015 [arXiv:1701.07782] [INSPIRE].

[11] B. Svetitsky, Looking behind the Standard Model with lattice gauge theory, EPJ Web Conf. 175 (2018) 01017 [arXiv: 1708.04840] [INSPIRE].

[12] O. Witzel, Review on composite Higgs models, PoS (LATTICE 2018)006 [arXiv:1901.08216] [INSPIRE].

[13] Z. Fodor et al., Nearly conformal gauge theories in finite volume, Phys. Lett. B 681 (2009) 353 [arXiv: 0907 .4562] [INSPIRE].

[14] V. Leino et al., The gradient flow running coupling in $\mathrm{SU}(2)$ gauge theory with $N_{f}=8$ fundamental flavors, Phys. Rev. D 95 (2017) 114516 [arXiv:1701.04666] [INSPIRE].

[15] V. Leino, K. Rummukainen and K. Tuominen, Slope of the $\beta$-function at the fixed point of $\mathrm{SU}(2)$ gauge theory with six or eight flavors, Phys. Rev. D 98 (2018) 054503 [arXiv: 1804.02319] [INSPIRE].

[16] V. Leino et al., Infrared behaviour of $\mathrm{SU}(2)$ gauge theory with $N_{f}$ fundamental flavours, in the proceedings of the $13^{\text {th }}$ Conference on Quark Confinement and the Hadron Spectrum (Confinement XIII), July 31-August 6, Maynooth, Ireland (2018), arXiv: 1811.12438 [INSPIRE].

[17] C.Y.H. Huang et al., Lattice study for conformal windows of $\mathrm{SU}(2)$ and $\mathrm{SU}(3)$ gauge theories with fundamental fermions, PoS(LATTICE 2015) 224 [arXiv:1511.01968] [INSPIRE]. 
[18] S.M. Nishigaki, P.H. Damgaard and T. Wettig, Smallest Dirac eigenvalue distribution from random matrix theory, Phys. Rev. D 58 (1998) 087704 [hep-th/9803007] [INSPIRE].

[19] P.H. Damgaard and S.M. Nishigaki, Distribution of the $k^{\text {th }}$ smallest Dirac operator eigenvalue, Phys. Rev. D 63 (2001) 045012 [hep-th/0006111] [INSPIRE].

[20] S.M. Nishigaki, Distribution of the $k^{\text {th }}$ smallest Dirac operator eigenvalue: an update, PoS(LATTICE 2015) 057 [arXiv: 1606.00276] [INSPIRE].

[21] J.J.M. Verbaarschot, The Spectrum of the QCD Dirac operator and chiral random matrix theory: The Threefold way, Phys. Rev. Lett. 72 (1994) 2531 [hep-th/9401059] [INSPIRE].

[22] M.L. Mehta, Random matrices, $3^{\text {rd }}$ edition, Elsevier, Amsterdam The Netherlands

[23] T. Nagao, Random matrices: an introduction (in Japanese), University of Tokyo Press, Tokyo Japan (2005).

[24] P.J. Forrester, Log-gases and random matrices, London Mathematical Society Monographs, Princeton University Press, Princeton U.S.A. (2010).

[25] S.M. Nishigaki, Random matrices and gauge theory (in Japanese), Science Press, Tokyo, Japan (2016).

[26] P.H. Damgaard and S.M. Nishigaki, Universal spectral correlators and massive Dirac operators, Nucl. Phys. B 518 (1998) 495 [hep-th/9711023] [INSPIRE].

[27] T. Wilke, T. Guhr and T. Wettig, The microscopic spectrum of the QCD Dirac operator with finite quark masses, Phys. Rev. D 57 (1998) 6486 [hep-th/9711057] [INSPIRE].

[28] F.J. Dyson, Statistical theory of the energy levels of complex systems. I, J. Math. Phys. 3 (1962) 140 [INSPIRE].

[29] T. Nagao and S.M. Nishigaki, Massive chiral random matrix ensembles at $\beta=1$ and $\beta=4$ : finite volume QCD partition functions, Phys. Rev. D 62 (2000) 065006 [hep-th/0001137] [INSPIRE].

[30] T. Nagao and S.M. Nishigaki, Massive chiral random matrix ensembles at $\beta=1$ and $\beta=4$ : QCD Dirac operator spectra, Phys. Rev. D 62 (2000) 065007 [hep-th/0003009] [INSPIRE].

[31] G. Akemann and E. Kanzieper, Spectra of massive and massless QCD Dirac operators: a novel link, Phys. Rev. Lett. 85 (2000) 1174 [hep-th/0001188] [INSPIRE].

[32] G. Akemann and E. Kanzieper, Spectra of massive QCD Dirac operators from random matrix theory: all three chiral symmetry breaking patterns, Nucl. Phys. Proc. Suppl. 94 (2001) 681 [hep-lat/0010092] [INSPIRE].

[33] A.D. Jackson, M.K. Sener and J.J.M. Verbaarschot, Finite volume partition functions and Itzykson-Zuber integrals, Phys. Lett. B 387 (1996) 355 [hep-th/9605183] [INSPIRE].

[34] P.J. Forrester, The spectrum edge of random matrix ensembles, Nucl. Phys. B 402 (1993) 709 [INSPIRE].

[35] M. Gaudin, Sur la loi limite de léspacement des valeurs propres d'une matrice aléatoire, Nucl. Phys. 25 (1961) 447.

[36] M.L. Mehta and J. des Cloizeaux, The probabilities for several consecutive eigenvalues of a random matrix, Indian J. Pure Appl. Phys. 3 (1970) 329.

[37] A. Edelman, Eigenvalues and condition numbers of random matrices, SIAM J. Matrix Anal. Appl. 9 (1988) 543. 
[38] A. Edelman, The distribution and moments of the smallest eigenvalue of a random matrix of wishart type, Lin. Alg. Appl. 159 (1991) 55.

[39] C.A. Tracy and H. Widom, Level spacing distributions and the Airy kernel, Phys. Lett. B 305 (1993) 115 [hep-th/9210074] [INSPIRE].

[40] C.A. Tracy and H. Widom, Level spacing distributions and the Bessel kernel, Commun. Math. Phys. 161 (1994) 289 [hep-th/9304063] [INSPIRE].

[41] P.J. Forrester and T. Hughes, Complex Wishart matrices and conductance in mesoscopic systems: Exact results, J. Math. Phys. 35 (1994) 6736.

[42] G. Akemann and P.H. Damgaard, Distributions of Dirac operator eigenvalues, Phys. Lett. B 583 (2004) 199 [hep-th/0311171] [INSPIRE].

[43] G. Akemann, J.C.R. Bloch, L. Shifrin and T. Wettig, Individual complex Dirac eigenvalue distributions from random matrix theory and lattice QCD at nonzero chemical potential, Phys. Rev. Lett. 100 (2008) 032002 [arXiv:0710.2865] [INSPIRE].

[44] G. Akemann and P.H. Damgaard, Individual eigenvalue distributions of chiral random two-matrix theory and the determination of $F(\pi)$, JHEP 03 (2008) 073 [arXiv:0803.1171] [INSPIRE].

[45] G. Akemann, M.J. Phillips and L. Shifrin, Gap probabilities in non-hermitian random matrix theory, J. Math. Phys. 50 (2009) 063504 [arXiv:0901.0897] [INSPIRE].

[46] G. Akemann and A.C. Ipsen, The $k^{\text {th }}$ smallest Dirac operator eigenvalue and the pion decay constant, J. Phys. A 45 (2012) 115205 [arXiv:1110.6774] [INSPIRE].

[47] G. Akemann and A.C. Ipsen, Individual eigenvalue distributions for the Wilson Dirac operator, JHEP 04 (2012) 102 [arXiv:1202.1241] [INSPIRE].

[48] T. Guhr and T. Wettig, An Itzykson-Zuber-like integral and diffusion for complex ordinary and supermatrices, J. Math. Phys. 37 (1996) 6395 [hep-th/9605110] [INSPIRE].

[49] W.H. Press et al., Numerical recipes in C, Cambridge University Press, Cambridge U.K. (1988).

[50] S.M. Nishigaki, Universality crossover between chiral random matrix ensembles and twisted SU(2) lattice Dirac spectra, Phys. Rev. D 86 (2012) 114505 [arXiv: 1208.3452] [INSPIRE].

[51] S.M. Nishigaki, Level spacings of parametric chiral random matrices and two-color QCD with twisted boundary condition, Prog. Theor. Phys. 128 (2012) 1283 [arXiv:1208.3878] [INSPIRE].

[52] S.M. Nishigaki and T. Yamamoto, Individual eigenvalue distributions for chGSE-chGUE crossover and determination of low-energy constants in two-color $Q C D+Q E D$, PoS (LATTICE 2014) 067 [arXiv:1501.07508] [INSPIRE].

[53] T. Yamamoto and S.M. Nishigaki, Individual eigenvalue distributions of crossover chiral random matrices and low-energy constants of $\mathrm{SU}(2) \times \mathrm{U}(1)$ lattice gauge theory, PTEP 2018 (2018) 023B01 [arXiv:1711.03388] [INSPIRE].

[54] P.J. Forrester and N.S. Witte, The distribution of the first eigenvalue spacing at the hard edge of the Laguerre unitary ensemble, Kyushu J. Math. 61(2) (2007) 457 [arXiv:0704.1926].

[55] N.S. Witte, F. Bornemann and P.J. Forrester, Joint distribution of the first and second eigenvalues at the soft edge of unitary ensembles, Nonlinearity 26 (2013) 1799 [arXiv:1209.2190]. 
[56] A. Borodin and A. Soshnikov, Janossy densities I. Determinantal ensembles, J. Stat. Phys. 113 (2003) 595 [math-ph/0212063].

[57] A. Soshnikov, Janossy densities II. Pfaffian ensembles, J. Stat. Phys. 113 (2003) 611 [math-ph/0301003].

[58] F. Bornemann, On the numerical evaluation of Fredholm determinants, Math. Comp. $\mathbf{7 9}$ (2010) 871 [arXiv:0804.2543].

[59] F. Bornemann, On the numerical evaluation of distributions in random matrix theory: a review, Markov Processes Relat. Fields 16 (2010) 803 [arXiv:0904.1581].

[60] C.Y.H. Huang, I. Kanamori, C.J.D. Lin and K. Ogawa, in preparation.

[61] APE collaboration, Glueball masses and string tension in lattice QCD, Phys. Lett. B 192 (1987) 163 [INSPIRE].

[62] G. Akemann, T. Guhr, M. Kieburg, R. Wegner and T. Wirtz, Completing the picture for the smallest eigenvalue of real Wishart matrices, Phys. Rev. Lett. 113 (2014) 250201 [Erratum ibid. 114 (2015) 179901] [arXiv: 1409.0360] [INSPIRE].

[63] T. Wirtz et al., The smallest eigenvalue distribution in the real Wishart-Laguerre ensemble with even topology, J. Phys. A 48 (2015) 245202 [arXiv:1502.03685] [INSPIRE].

[64] S. Sachdev and J. Ye, Gapless spin fluid ground state in a random, quantum Heisenberg magnet, Phys. Rev. Lett. 70 (1993) 3339 [cond-mat/9212030] [INSPIRE].

[65] A. Kitaev, A simple model of quantum holography, talks given at KITP, April 7 and May 27 (2015).

[66] V. Rosenhaus, An introduction to the SYK model, arXiv:1807.03334 [INSPIRE].

[67] W. Fu, D. Gaiotto, J. Maldacena and S. Sachdev, Supersymmetric Sachdev-Ye-Kitaev models, Phys. Rev. D 95 (2017) 026009 [arXiv: 1610.08917] [InSPIRE].

[68] A.M. García-García, Y. Jia and J.J.M. Verbaarschot, Universality and Thouless energy in the supersymmetric Sachdev-Ye-Kitaev Model, Phys. Rev. D 97 (2018) 106003 [arXiv: 1801.01071] [INSPIRE].

[69] H. Leutwyler and A.V. Smilga, Spectrum of Dirac operator and role of winding number in QCD, Phys. Rev. D 46 (1992) 5607 [inSPIRE].

[70] A. Soshnikov, Determinantal random point fields, Russ. Math. Surv. 55(5) (2007) 923 [math/0002099].

[71] R. Lyons, Determinantal probability measures, Publ. Math. Inst. Hautes Et. Sci. 98 (2003) 167 [math/0204325].

[72] J. Ben Hough, M. Krishnapur, Y. Peres and B. Virág, Determinantal processes and independence, Prob. Surv. 3 (2006) 206 [math/0503110].

[73] O. Macchi, The coincidence approach to stochastic point processes, Adv. Appl. Prob. 7 (1975) 83.

[74] A. Borodin and G. Olshanski, Distributions on partitions, point processes, and the hypergeometric kernel, Commun. Math. Phys. 211 (2000) 335.

[75] S. Duane, A.D. Kennedy, B.J. Pendleton and D. Roweth, Hybrid Monte Carlo, Phys. Lett. B 195 (1987) 216 [INSPIRE]. 